\title{
6 Der institutionelle Rahmen für die Wahrnehmung von Verantwortung
}

\subsection{Einführung}

Zur Erinnerung: Für die Überwindung der sich abzeichnenden Ziel- und Verteilungskonflikte sind die Steuerungswirkungen der zur Verfügung stehenden Instrumente nur bedingt geeignet (s. Kap. 1.2). Es bedarf deshalb zu deren Ergänzung der Etablierung von Verantwortungsbewusstsein.

Wie dieses erreicht werden kann, ist Gegenstand der nun folgenden Kapiteln 6 und 7. Dabei werden drei Komponenten unterschieden (in Anlehnung an Göbel 2006, S. 109ff.):

1. Von zentraler Bedeutung für das Wirksamwerden der neuen Unternehmensstrategie sind die Fähigkeiten der Geschäftsführung und die Rolle, die diese künftig spielen wird. Die Geschäftsführung muss von der Notwendigkeit und dem Nutzen eines Managements der sozialen Verantwortung überzeugt sein. Sie muss bereit und in der Lage sein, dieses konsequent gegenüber allen Verantwortungsträgern und Stakeholdern zu vertreten (s. Kap. 6.3) (Cöbel 1992, S. 268).

2. Es werden ein Unternehmensleitbild und darin eingeschlossen ein Führungskonzept gebraucht, mit deren Hilfe den Verantwortungsträgern gesagt wird, was von ihnen erwartet wird, und das die Voraussetzungen dafür schafft, dass die Verantwortungsträger die ihnen zugeschriebene Verantwortung wahrnehmen wollen und können (s. Kap. 7).

3. Eine weitere Voraussetzung für das Wahrnehmen von Verantwortung ist die dafür geeignete Gestaltung der Unternehmensstruktur des Krankenhauses. Damit diese das Praktizieren der Goldenen Regel nachhaltig unterstützen kann, werden bei seiner Entwicklung Stakeholder-Anliegen in zwei Ebenen in Betracht gezogen (Göbel 2006, S. 233f.):

- Einerseits geht es bei der Entwicklung der Unternehmensstruktur vor allem darum, organisationale Barrieren, die das Wahrnehmen von Verantwortung, also das Berücksichtigen von Stakeholder-Bedürfnissen, behindern könnten, zu vermeiden bzw. abzubauen.

- Zum anderen gilt es, bei diesem Prozess der Arbeitsplatzgestaltung die Bedürfnisse der Verantwortungsträger, zu deren Unterstützung der institutionelle Rahmen dienen soll, angemessen zu berücksichtigen. Damit wird die Voraussetzung dafür geschaffen, dass sich die Verantwortungsträger im Unternehmen, die auch Stakeholder sind, mit der neuen Unternehmensstrategie identifizieren können und dass sie ausrei- 
chend motiviert sind, sich des institutionellen Rahmens zur Wahrnehmung von Verantwortung bedienen zu wollen.

In den weiteren Ausführungen werden unter der Unternehmensstruktur (s. Abb. 35) die Leitungsstruktur des Krankenhauses (s. Kap. 6.4) und der Prozess der betrieblichen Leitungsentscheidung (s. Kap. 6.5) verstanden.

Bevor die Entwicklung und Implementierung einer geeigneten Unternehmensstruktur und eines geeigneten Unternehmensleitbildes erörtert werden, soll zunächst eine Antwort auf die Frage gesucht werden (s. Kap. 6.2), anhand welcher Kriterien man erkennen kann, ob überhaupt und in welchem Maße die Ideen des Managements der sozialen Verantwortung umgesetzt worden sind und praktiziert werden.

\subsection{Grundpostulate für ein Management der sozialen Verantwortung}

\subsubsection{Einführung}

Damit das Schaffen des Unternehmensleitbildes und das der Unternehmensstruktur auch im Detail aufeinander abgestimmt erfolgt und die $\mathrm{Ge}-$ staltungsergebnisse nicht den Charakter von Beliebigkeit haben, muss sich deren Entwicklung von klaren Grundsätzen (s. Tab. 11 - in Anlehnung an Scholz, S. 65ff. und Steinmann; Löhr (1991), S. 1off.) leiten lassen. Letztere werden aus der Grundidee des Managements der sozialen Verantwortung abgeleitet. Anhand des Erfüllungsgrades dieser Normen lässt sich beurteilen, ob überhaupt und in welchem Ausmaß das Management der sozialen Verantwortung in den Alltag des Krankenhauses umgesetzt worden ist und gelebt wird.

Die Beschreibung der Grundpostulate und deren Einfluss auf die Gestaltung der Unternehmensstruktur erfolgt in zwei Stufen:

1. Zunächst werden die Crundpostulate skizziert (s. Kap. 6.2.4 bis 6.2.10),

2. um im Anschluss daran im Konkreten und im Detail zu zeigen, wie die Unternehmensstruktur (s. Kap. 6.4 und 6.5) und das Unternehmensleitbild (s. Kap. 7) unter Berücksichtigung der Crundpostulate idealerweise so gestaltet werden, dass organisationale Barrieren die Wahrnehmung von Verantwortung nicht behindern und Stakeholder-Bedürfnisse bestmöglich befriedigt werden können.
Tab. 11 Grundpostulate des Managements der sozialen Verantwortung

Grundpostulate

Erfolgsorientierung

Berücksichtigung der Interessen Betroffener

Förderung der argumentativen, dialogischen

Verständigung

Förderung der Mündigkeit der Stakeholder

Beachtung moralischer Prinzipien

Beschränkung des Gewinnprinzips

Begründungspflicht

\subsubsection{Die Entwicklung der Grundpostulate}

Für die Spezifizierung der Crundpostulate und für die Bestimmung der Gewichte, mit denen die Grundpostulate bei der Gestaltung der Unternehmensstruktur und des Unternehmensleitbildes berücksichtigt werden sollen, sind bestimmte Regeln einzuhalten. Diese werden in Kapitel 7 ausführlich erörtert. Hier soll nur darauf hingewiesen werden, dass sowohl die GrundpostulateSpezifizierung als auch die Gewichte-Festlegung unter Berücksichtigung der im Regelfall schon vor dem Beginn dieses Prozesses - vielfach allerdings unbewusst - angewendeten Crundpostulaten erfolgen sollten. So haben zum Beispiel die Mitarbeiter das Bedürfnis, an der Entwicklung der Grundpostulate und deren Anwendungs-Cewichte im Sinne einer argumentativen, dialogischen Verständigung beteiligt zu werden. Sie wollen auf diese Weise - indem sie ihre Bedürfnisse an die Gestaltung der Grundpostulate geltend machen sicherstellen, dass Grundpostulate entstehen, die ihren Bedürfnissen entsprechen und die sie in der Praxis erfolgreich anwenden können.

\subsubsection{Die Rolle der Grundpostulate bei der Gestaltung der Unternehmensstruktur}

Die Unternehmensstruktur dient der Realisierung der Unternehmensziele und bedarf deshalb einer spezifischen Gestaltung. Sie wird zum anderen so ausgerichtet, dass organisationale Barrieren die Wahrnehmung von Verantwortung nicht behindern. 


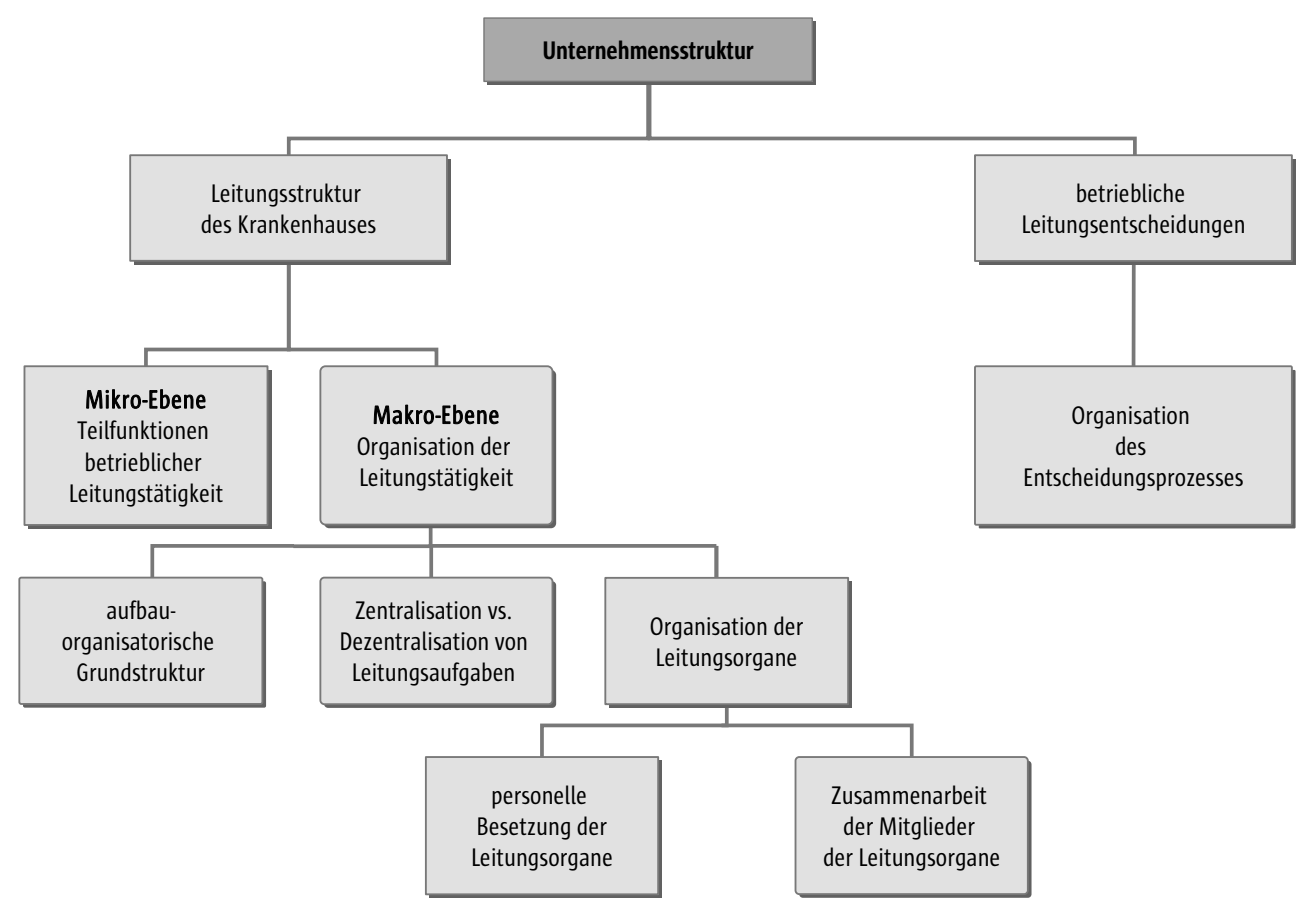

Abb. 35 Unternehmensstruktur

So gesehen spielen die Grundpostulate bei der Gestaltung der Unternehmensstruktur in mehrfacher Hinsicht eine Rolle:

1. Gemeinsam mit den Stakeholder-Bedürfnissen dienen sie als Normen für die Gestaltung der Unternehmensstruktur.

2. Sie werden benötigt bei der Entscheidung, ob eine Gestaltungsalternative im Sinne des Managements der sozialen Verantwortung überhaupt in Betracht gezogen werden kann;

3. Die Grundpostulate werden durch die Geschäftsführungen unterschiedlich gewichtet. Das jeweils festgelegte Gewicht bestimmt die Auswahl unter mehreren zur Verfügung stehenden Alternativen zur Gestaltung der Unternehmensstruktur.

\section{Zu 1. Grundpostulate und Stakeholder-Bedürfnisse als Gestaltungsnormen}

Anhand eines Beispiels (s. Beispiel 1) wird der Zusammenhang zwischen

- Grundpostulaten,

- Stakeholder-Bedürfnissen,

- Anforderungen an die Gestaltung der Unternehmensstruktur und
- Folgen der Stakeholder-Bedürfnis-Befriedigung dargestellt.

Es werden die Indikatoren genannt, die zum Messen des Grades der Stakeholder-Bedürfnis-Befriedigung eingesetzt werden.

\section{Beispiel 1 (s. auch Tab. 12 und Abb. 36)}

Nachdem die Geschäftsführung festgelegt hat, dass die Prozesse der Personalauswahl und der -entwicklung unter Berücksichtigung der Grundideen des Managements der sozialen Verantwortung gestaltet werden sollen, schließt sich zur Lösung dieses Problems die folgende Vorgehensweise an (die Nummerierung der folgenden Absätze entspricht der Nummerierung der Spalten in Tabelle 12):

1. Es wird zunächst geprüft, welche Grundpostulate gegebenenfalls zu beachten sind. Im Beispiel ist es unter anderem das Grundpostulat „Berücksichtigung der Interessen Betroffener". Diesem Grundpostulat wird dadurch entsprochen, dass die betroffenen Mitarbeiter an der Gestaltung der ausgewählten Prozesse beteiligt werden.

2. Danach werden die Bedürfnisse der betroffenen Stakeholder in Bezug auf die Folgen, die als Ergebnis der zu fällenden Personalentscheidungen zu erwarten sind, identi- 
Tab. 12 Beispiel für den Zusammenhang zwischen Grundpostulat, Mitarbeiter-Bedürfnis, Anforderungen an die Unternehmensstruktur und dem Messen der Folgen der Mitarbeiter-Bedürfnis-Befriedigung

\begin{tabular}{|c|c|c|c|c|}
\hline Grundpostulat & $\begin{array}{l}\text { Mitarbeiter- } \\
\text { Bedürfnis }\end{array}$ & $\begin{array}{l}\text { Folgen der Bedürfnis- } \\
\text { Befriedigung (u.a.) }\end{array}$ & Indikatoren (Beispiele) & $\begin{array}{l}\text { Anforderungen an das } \\
\text { Personalmanagement }\end{array}$ \\
\hline 1. & 2. & 3. & 4. & 5. \\
\hline $\begin{array}{l}\text { Berücksichtigung } \\
\text { der Interessen } \\
\text { Betroffener durch } \\
\text { Beteiligung an } \\
\text { der Entwicklung } \\
\text { und Implementie- } \\
\text { rung der Personal- } \\
\text { management- } \\
\text { Instrumente }\end{array}$ & $\begin{array}{l}\text { Bedürfnisses } \\
\text { nach Sicherheit } \\
\text { im Sinne des } \\
\text { zeit- und sach- } \\
\text { gerechten Bewäl- } \\
\text { tigenkönnens der } \\
\text { den Mitarbeitern } \\
\text { übertragenen } \\
\text { Aufgaben }\end{array}$ & $\begin{array}{l}\text { vorhandene Mitarbei- } \\
\text { ter nehmen die wahr- } \\
\text { zunehmende Arbeit } \\
\text { als zufriedenstellend, } \\
\text { zumutbar, erträg- } \\
\text { lich und ausführbar } \\
\text { (in dieser Rangfolge) } \\
\text { wahr (Scholz, S. 578; } \\
\text { Rohmert, S. 64) und } \\
\text { werden deshalb län- } \\
\text { ger an den Arbeitge- } \\
\text { ber gebunden } \\
\text { Personalbeschaffung } \\
\text { wird positiv beein- } \\
\text { flusst } \\
\text {.... }\end{array}$ & $\begin{array}{l}\text { Dauer der Betriebszugehörigkeit } \\
\text { Fluktuationsrate } \\
\text { Krankenstand } \\
\text { Anzahl der Bewerber je aus- } \\
\text { geschriebene Stelle aus dem } \\
\text { internen Arbeitsmarkt } \\
\text {... } \\
\text { Anzahl der Bewerber je aus- } \\
\text { geschriebene Stelle } \\
\text { sach- und zeitgerechte Besetzung } \\
\text { einer freien Stelle } \\
\text { Anteil der Bewerber an der } \\
\text { Gesamtzahl der Bewerber, die } \\
\text { zu einem Vorstellungsgespräch } \\
\text { eingeladen werden } \\
\text {... }\end{array}$ & $\begin{array}{l}\text { Personalauswahl wird } \\
\text { so gestaltet, dass die } \\
\text { Rekrutierung entspre- } \\
\text { chend qualifizierter } \\
\text { Mitarbeiter sicher- } \\
\text { gestellt werden kann } \\
\text { Personalentwicklung } \\
\text { stellt die bedarfs- } \\
\text { gerechte Weiterent- } \\
\text { wicklung vorhandener } \\
\text { Mitarbeiter sicher } \\
\text {... }\end{array}$ \\
\hline
\end{tabular}

fiziert. Im Beispiel ist es das „Bedürfnis nach Sicherheit“; dabei wird unter Sicherheit das zielkonforme Bewältigenkönnen der übertragenen Aufgaben verstanden.

3. Es empfiehlt sich, die Folgen zu beschreiben, die sich als Ergebnis der Personalentscheidungen für die Mitarbeiter einstellen können, und

4. die Indikatoren zu bestimmen, anhand derer die Bedürfnis-Befriedigung gemessen werden kann.

5. Danach erfolgt die konkrete Gestaltung der ausgewählten Prozesse. Diese werden unter Berücksichtigung der Mitarbeiter-Bedürfnisse so modelliert, dass nur noch Mitarbeiter eingestellt werden, von denen angenommen werden kann, dass sie sich mit den Grundprinzipien des Managements der sozialen Verantwortung identifizieren und diese anwenden wollen und können. Vorhandene Mitarbeiter werden - sofern notwendig so weiter qualifiziert, dass sie die ihnen übertragenen Aufgaben im Sinne der Goldenen Regel anforderungsgerecht ausführen können.

\section{Zu 2. Auswahl der in Betracht zu ziehenden Gestaltungsalternativen}

Für die mit dem Ziel der Realisierung bestimmter Unternehmensziele vorzunehmende Gestaltung der Unternehmensstruktur und/oder der Struktur-Komponenten stehen im Regelfall mehrere
Alternativen zur Verfügung. Bei der Auswahl der unter Verwendung der Crundpostulate überhaupt in Betracht zu ziehenden Gestaltungsalternativen kann ein Entscheidungsbaum (s. Abb. 37 und Beispiel 2) eine Hilfe sein.

\section{Beispiel 2}

Wenn Mitarbeiter in auseichender Zahl zur Verfügung stehen, diese aber nur zu einem Teil den Anforderungen des Managements der sozialen Verantwortung entsprechen, bieten sich der Geschäftsführung, bezogen auf die nicht ausreichend qualifizierten Mitarbeiter, zwei Alternativen zur Lösung des Problems an:

1. Die Mitarbeiter werden entsprechend den Anforderungen des Managements der sozialen Verantwortung qualifiziert - sofern sie über die erforderlichen Entwicklungspotenziale verfügen.

2. Die nicht ausreichend qualifizierten Mitarbeiter werden freigesetzt. Neue Mitarbeiter mit der erforderlichen Einstellung und den notwendigen Kenntnissen und Fähigkeiten werden rekrutiert.

Die Geschäftsführung bewertet die beiden Alternativen anhand der Grundpostulate. 


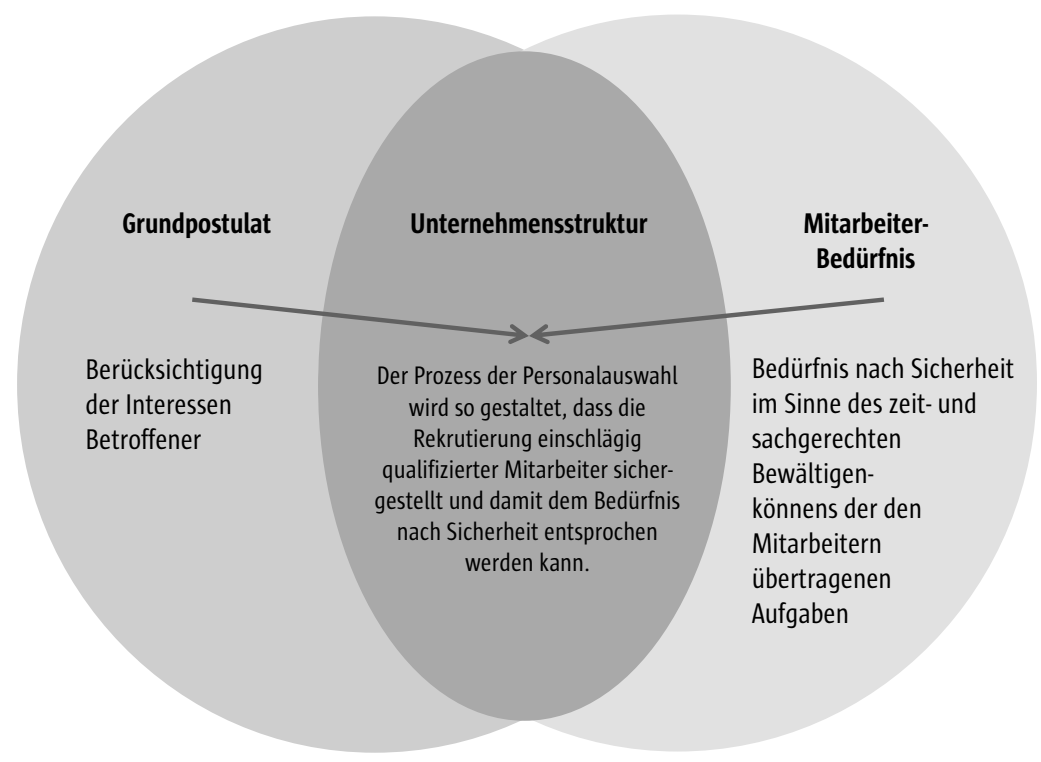

Abb. 36 Zusammenhang - beispielhaft - zwischen Grundpostulaten, Mitarbeiter-Bedürfnis und Unternehmensstruktur

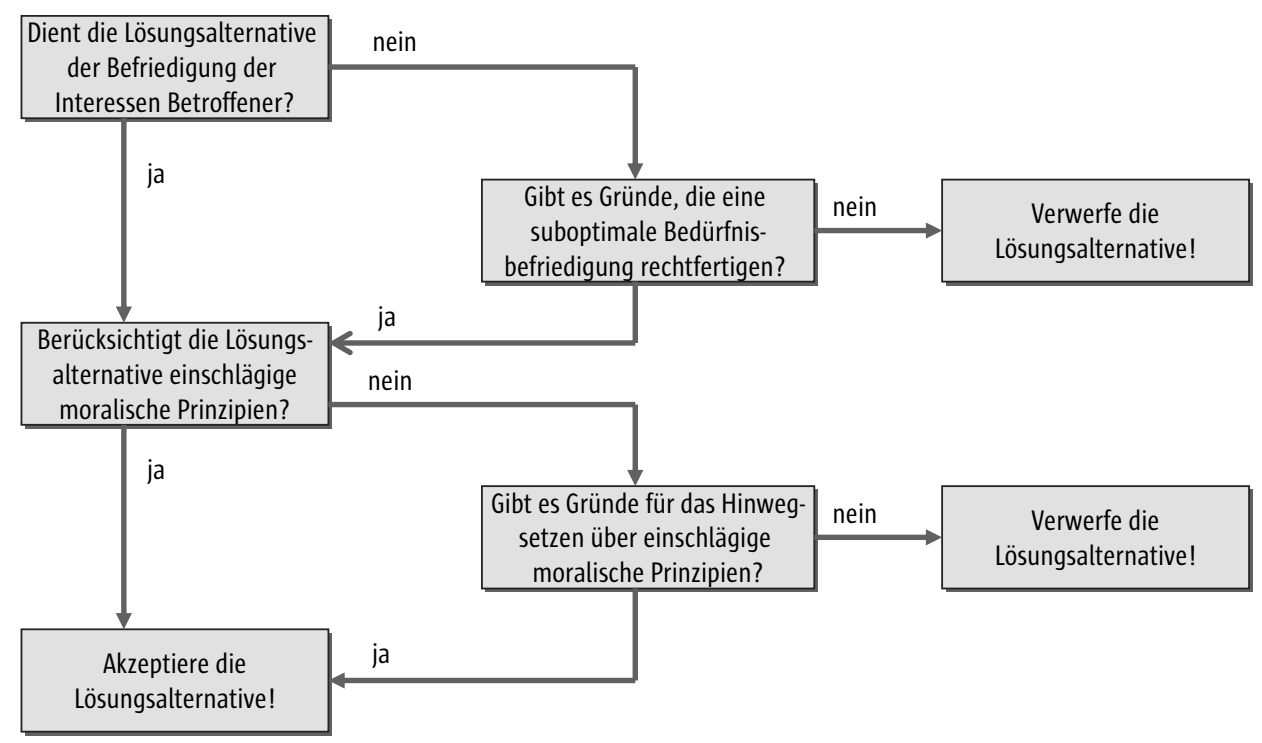

Abb. 37 Entscheidungsbaum für die Berücksichtigung von Grundpostulaten (in Anlehnung an Grabner-Kräuter, S. 223 und die dort genannte Literatur)

\section{Zu 3. Gewichtung der Grundpostulate zwecks Entscheidung zwischen verschiedenen Gestaltungsalternativen}

In den Krankenhäusern spielen die Grundpostulate bei der Gestaltung der Unternehmensstruk- tur eine jeweils unterschiedlich gewichtige Rolle. So wird in einem Krankenhaus in privater Trägerschaft das Grundpostulat „Beschränkung des Gewinnprinzips" bei der Beantwortung der Frage, wie die Unternehmensstruktur des Kran- 
kenhauses beschaffen sein soll, vielleicht weniger bedeutsam sein als in einem Krankenhaus in gemeinnütziger Trägerschaft. Dagegen wird das Grundpostulat „Beachtung moralischer Prinzipien“ in dem zuletzt genannten Krankenhaus bei der Festlegung der Unternehmensstruktur von eher größerer Bedeutung sein. Die Unternehmensstrukturen werden deshalb in den beiden Arten von Krankenhäusern möglicherweise unterschiedlich gestaltet sein. Die Festlegung der Unternehmensstruktur setzt deshalb voraus, dass diejenigen, die die Bewertung der Alternativen für die Gestaltung der Unternehmensstruktur vornehmen wollen, sich darüber verständigen, mit welchem Gewicht die verschiedenen Grundpostulate bei der Bewertung berücksichtigt werden sollen (s. Abb. 38).

\subsubsection{Erfolgsorientierung}

Das Management der sozialen Verantwortung darf nicht Selbstzweck sein. Der durch dessen Realisierung verursachte Aufwand muss durch einen entsprechend hohen Nutzen gerechtfertigt werden.

Unter dem Erfolg des Managements der sozialen Verantwortung kann man zweierlei verstehen:
1. Einerseits leistet die Realisierung des Managements der sozialen Verantwortung einen Beitrag zum Erreichen der Unternehmensziele, also unter anderem zur Sicherung der Existenz des Krankenhauses und der darin vorgehaltenen Arbeitsplätze sowie zur weiteren Verbesserung der Qualität der Patientenbehandlung. Sozial verantwortliches Handeln trägt dazu bei, dass die als legitim akzeptierten Bedürfnisse diverser Stakeholder - auch zum Nutzen des Krankenhauses - befriedigt werden.

2. Unter dem Erfolg des Managements der sozialen Verantwortung wird - gewissermaßen als Voraussetzung für die Realisierung der unter Ziffer 1. genannten Ziele - auch das konsequente Handeln der Geschäftsführung und der übrigen Verantwortlichen im Sinne der Goldenen Regel verstanden.

Die zuletzt genannte Definition der Erfolgsorientierung wird im Rahmen der vorliegenden Untersuchung zum Thema gemacht. Die Strukturen des Krankenhauses werden daraufhin überprüft, ob sie geeignet sind, die Verantwortlichen bei der Wahrnehmung der Verpflichtungen, die sich aus der Grundidee des Managements der sozialen Verantwortung ergeben, zu unterstützen.

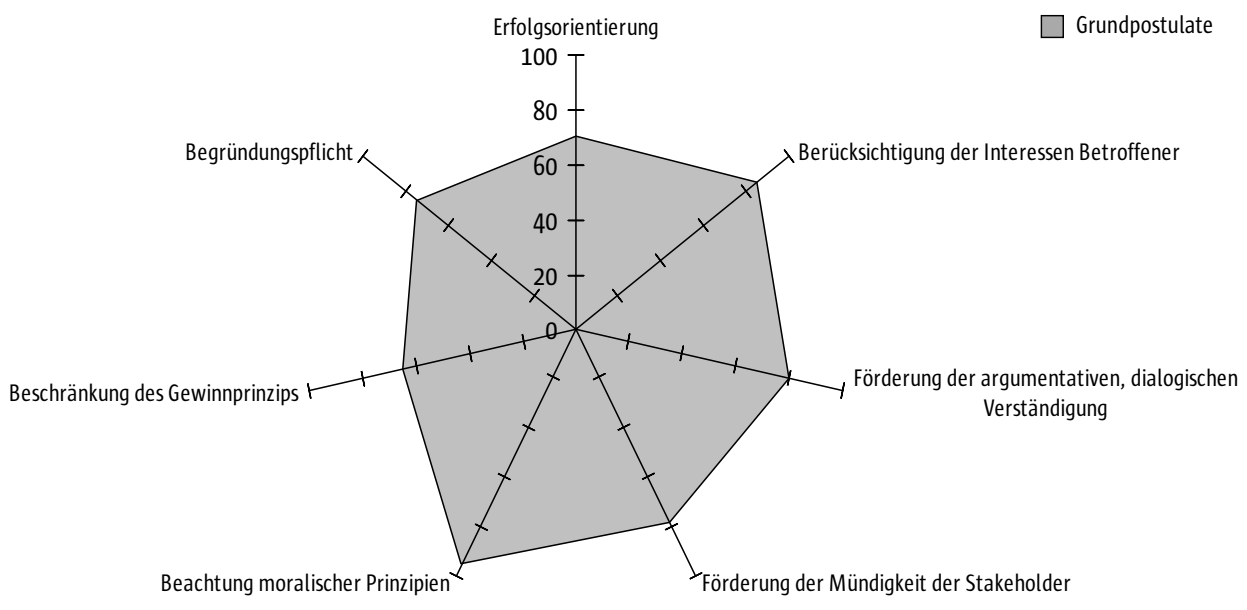

Abb. 38 Gewichtung der Grundpostulate als Basis für die Bewertung der Unternehmensstruktur-Gestaltungs-Alternativen (Beispiel) 


\subsubsection{Berücksichtigung der Interessen Betroffener}

Die Geschäftsführung eines Krankenhauses fällt Entscheidungen und setzt diese durch konkretes Handeln um. Das Umsetzen dieser Entscheidungen sollte die als legitim akzeptierten Stakeholder-Anliegen befriedigen. Die Folgen können wenn das Grundpostulat „Berücksichtigung der Interessen Betroffener" nicht angemessen berücksichtigt wird - sein, dass Rechte Betroffener verletzt und/oder deren Entscheidungsspielraum, den sie zwecks Realisierung ihrer eigenen Ziele benötigen, eingeschränkt wird.

\section{Beispiel}

Von dem Ergebnis der Unternehmensplanung im Allgemeinen und dem der Personalbedarfsbestimmung im Besonderen sind unterschiedliche Stakeholder betroffen:

Die Patienten wollen ohne warten zu müssen von bestens qualifizierten und von einer ausreichend hohen Zahl von Ärzten, Pflegkräften und Therapeuten erfolgreich behandelt werden. Wenn dieses Bedürfnis nicht befriedigt wird, müssen die Patienten hinnehmen, dass sie länger als medizinisch geboten im Krankenhaus bleiben müssen.

Die Mitarbeiter erwarten eine Personalausstattung, die ihnen die Bewältigung der ihnen übertragenen Aufgaben ohne ständige Überbelastung erlaubt und die eine Dienstplangestaltung mit einem angemessenen Ausgleich zwischen betrieblichen und privaten Interessen ermöglicht. Wenn das zuletzt genannte Bedürfnis nicht befriedigt wird, können die Mitarbeiter ihrer Verantwortung gegenüber ihrer Familie möglicherweise nicht nachkommen.

Der Eigentümer des Krankenhauses beansprucht eine marktgerechte Verzinsung des von ihm zur Verfügung gestellten Kapitals und ist deshalb an einer an Produktivitätskriterien orientierten Personalausstattung interessiert. Die Nicht-Befriedigung dieses Bedürfnisses führt möglicherweise dazu, dass der Eigentümer seinen bisher gewohnten Lebensstandard einschränken muss.

Zwischen den Anliegen der Stakeholder bestehen möglicherweise Konkurrenzbeziehungen. Insbesondere kann eine solche Beziehung zwischen den Bedürfnissen der Patienten und der Mitarbeiter auf der einen und der Gewinnerzielungsabsicht des Eigentümers auf der anderen Seite vermutet werden.

Um die sich dadurch abzeichnenden Konflikte lösen zu können, verpflichtet sich die Geschäftsführung, die Bedürfnisse der Stakeholder proaktiv und systematisch zu analysieren und zu bewerten, um sie bei ihren Entscheidungen angemessen, das heißt ausgewogen berücksichtigen zu können. Das Krankenhaus legt in einem Unternehmensleitbild fest, die Folgen seines Handelns jeweils daraufhin zu überprüfen, ob diese im Sinne der Goldenen Regel gerechtfertigt werden können. Es verpflichtet sich, Handlungen möglichst zu unterlassen, die den Anliegen der Betroffenen entgegenstehen und/oder deren Spielraum für die Realisierung ihrer eigenen Ziele allzu sehr einengen.

Prozesse unternehmerischer Entscheidungen, wie der als Beispiel verwendete Prozess der Personalbedarfsbestimmung, werden so organisiert, dass sich möglichst alle die von dem Ergebnis der Entscheidung betroffenen Stakeholder - entweder persönlich oder durch Agenten vertreten - daran beteiligen können, um ihre Interessen geltend zu machen. Dazu gehört auch, dass allen an der Entscheidung Beteiligten rechtzeitig alle entscheidungsrelevanten Materialien zur Verfügung gestellt werden (Wittmann, S. 95 und 102ff.).

\subsubsection{Förderung der argumentativen, dialogischen Verständigung}

Die Berücksichtigung der Interessen Betroffener setzt nicht nur die adäquate Organisation der Entscheidungsprozesse voraus. Das Crundpostulat „Berücksichtigung der Interessen Betroffener“ kann vielmehr nur dann erfüllt werden, wenn einige Bedingungen erfüllt sind:

- Erstens wird vorausgesetzt, dass diejenigen, die die Entscheidungen zu fällen haben, zur Offenheit zum Dialog, zur argumentativen Auseinandersetzung mit den Stakeholdern und zur ethischen Reflexion ihres Handelns imstande und bereit sind. Sie lassen ihre Meinung und ihre Ansprüche in Frage stellen. Sie können ihre Argumente in einer Sprache vortragen, die von den Betroffenen und von den an dem Entscheidungsprozess sonst noch Beteiligten verstanden werden kann. Das Krankenhaus verpflichtet sich deshalb, in die einschlägige Weiterentwicklung der Geschäftsführung, der Führungskräfte und der Mitarbeiter zu investieren.

- Zweitens wird von denjenigen, die von den Entscheidungen betroffen sind, erwartet, dass sie imstande und bereit sind, ihre Anliegen zu begründen. Sie sollten sachkundig genug sein, 
um sich mit den Argumenten der Verantwortungsträger erfolgreich auseinandersetzen zu können.

Der Dialog mit den von den Entscheidungen der Verantwortungsträger betroffenen ist für das Wirksamwerden des Managements der sozialen Verantwortung und damit auch für die Berücksichtigung der Interessen der von unternehmerischen Entscheidungen betroffenen aus weiteren Cründen von Bedeutung (Göbel 1992, S. 17off.):

- Die Stakeholder sind die beste Datenquelle, um die für das Unternehmen relevanten Stakeholder identifizieren und charakterisieren sowie um deren Anliegen analysieren und bewerten zu können. Der Dialog bietet die beste Gewähr für die Validität der Daten. Er schützt den Verantwortungsträger davor, eigene - und möglicherweise nicht zutreffende - Einschätzungen zur Grundlage von Entscheidungen zu machen.

- Der Dialog mit den Stakeholdern dient dem Finden von Normen, von Leitlinien, die von allen Beteiligten als Grundlage unternehmerischer Entscheidungen akzeptiert werden können.

Möglicherweise lässt sich dieses Ziel nicht sofort erreichen. Dann ist es wichtig, dass sich die Beteiligten darauf verständigen, wie sie vorgehen wollen, um verbindliche Normen entwickeln zu können.

- Der Dialog ist eine vertrauensbildende Maßnahme. Stakeholder nehmen die Bereitschaft zum Dialog mit ihnen als Wertschätzung wahr. Die für Stakeholder negativen Entscheidungen werden von diesen als Ergebnis eines Dialogs eher akzeptiert.

- Der Dialog eröffnet die Möglichkeit, die Einflussnahme der Stakeholder auf die unternehmerischen Entscheidungen zu steuern.

Nicht alle Stakeholder sind in der Lage, ihre Interessen persönlich zu vertreten. Die Interessen werden deshalb nicht nur direkt, sondern auch über vermittelnde Zwischenstufen - zum Beispiel über Politiker, Verbände, Personalvertretungen und Kammern - an die Verantwortungsträger herangetragen. Diese Agenten werden im Regelfall nicht für einzelne Stakeholder, sondern eher für eine Gruppe von ihnen tätig. Deshalb kann nicht immer gewährleistet werden, dass das individuelle Anliegen eines einzelnen Stakeholders in seiner ursprünglichen Ausprägung als Grundlage einer unternehmerischen Entscheidung Berücksichtigung findet.

Die Entwicklung von Normen wird durch unternehmensinterne und -externe Faktoren beeinflusst. Diese können sich im Zeitablauf ändern. Normen müssen deshalb modifizierbar sein. Die Geschäftsführung ist deshalb aufgefordert, die Voraussetzungen dafür zu schaffen, dass der Dialog überhaupt stattfinden kann; er muss auch in die Zukunft hinein offen gehalten werden (Steinmann; Löhr, S. 12).

\subsubsection{Förderung der Mündigkeit der Stakeholder}

Die argumentative, dialogische Verständigung zwischen den Verantwortungsträgern und denjenigen, die von Entscheidungen betroffen sind, setzt nicht nur das Erfüllen struktureller und prozessualer Normen und einschlägige soziale Kompetenz bei den Entscheidungsträgern voraus. Damit die Stakeholder - und das gilt insbesondere für die Führungskräfte und für die Mitarbeiter in ihrer Rolle als Stakeholder, aber nicht nur für diese - ihre Interessen mit Erfolg geltend machen können, sind diese auf der Basis einer kritischen Loyalität zu den Aktivitäten des Krankenhauses imstande, unternehmensintern für richtig gehaltene Definitionen kritisch in Frage zu stellen und differente Wahrnehmungen und für richtig gehaltene Revisionen auch gegen Widerstand zur Geltung zu bringen (Wittmann, S. 128).

Die Geschäftsführung verpflichtet sich deshalb, ein Betriebsklima zu schaffen, das die Bereitschaft der Stakeholder zur Präsentation ihrer Anliegen und zur Offenlegung von Entwicklungen, die von diesen als Fehlentwicklungen wahrgenommen werden, fördert und das zu der Gewissheit beiträgt, dass diese Offenheit nicht schadet und dass Willkür bei der Bewertung der von Stakeholdern gegebenen Hinweise ausgeschlossen ist ${ }^{49}$. Von zentraler Bedeutung in diesem Zusammenhang ist die Entwicklung einer Fehlerkultur, die Fehler nicht primär personalisiert, sondern vor allem und zuerst als Chance für die kontinuierliche Verbesserung von Strukturen

49 So ist zum Beispiel immer wieder von Patienten zu hören, dass diese als für sie nachteilig empfundene Entwicklungen nicht zur Sprache bringen, weil sie fürchten, weitere Nachteile während des noch andauernden und/oder eines erneuten Aufenthaltes im Krankenhaus in Kauf nehmen zu müssen. 
und Prozessen und in deren Folge von Ergebnissen begreift ${ }^{50}$. Die Mitarbeiter dürfen nicht mehr als „Fehlerquelle“, sie sollten als „Lösungsquelle“ angesehen werden (KdA, S. 1).

\subsubsection{Beachtung moralischer Prinzipien}

Angesichts zunehmender Spezialisierung und Arbeitsteilung im Allgemeinen und im Krankenhaus im Besonderen werden

1. die Handlungsketten immer länger und

2. die Zahl der Entscheidungsträger, die die Folgen der gefällten Entscheidung durch ihre Handlungen beeinflussen können, immer größer.

So kommt es, dass immer mehr Entscheidungen gefällt werden, deren Handlungsfolgen nicht mit Sicherheit vorhersehbar sind und die zu Folgen führen können, die von der Instanz, die das Unternehmensziel formuliert hat, so nicht gewollt waren. Die Geschäftsführung kann nicht darauf vertrauen, dass der Planungsprozess in allen seinen Verästelungen über das Unternehmensziel allein beherrscht werden kann (Schreyögg 1991, S. 262). Das Erfüllen der bisher skizzierten Kriterien allein ist als Voraussetzung für verantwortliches Handeln nicht ausreichend.

Es ist notwendig, neben der Bewertung unternehmerischen Handelns anhand der Folgen auch das Pflichtgemäße als Bewertungsmaßstab zu berücksichtigen (Göbel 2006, S. 27). Die Geschäftsführung verpflichtet sich und alle diejenigen, die befugt sind, unternehmerische Entscheidungen zu fällen, das, was aktuell in der Gesellschaft als Handlung, Zustand oder Haltung für gut oder wünschenswert bzw. für böse und verboten gehalten wird, als Handlungsnormen in Betracht zu ziehen (Göbel 2006, S. 7).

Damit können Handlungsfolgen, die von einem Verantwortungsträger nicht intendiert waren und aus der Sicht der Betroffenen negativ bewertet werden, nicht gänzlich verhindert werden. Schlechte Folgen werden aber, wenn auch der gute Wille und die gute Tat bei der Bewertung der Leistung des Entscheidungsträgers bedacht

50 Von einer zunehmenden Zahl von Krankenhäusern wird - gewissermaßen als Hilfestellung - das Critical Incident Reporting-System(CIRS), ein Berichtssystem zur - meist anonymen - Meldung von kritischen Ereignissen und Beinahe-Schäden, eingesetzt. werden, diesem nicht im Sinne des Ergebnisses schuldhaften Verhaltens zugerechnet (Göbel 2006, S. 27).

\subsubsection{Beschränkung des Gewinnprinzips}

Die Gewinnerzielung wird als notwendige Voraussetzung zur Übernahme von sozialer Verantwortung, also zur Berücksichtigung der Stakeholder-Interessen, angesehen. Wenn das Krankenhaus Gewinne nicht erzielen kann, ist es nicht imstande, in die personelle, technische und organisatorische Weiterentwicklung der Patientenbehandlung und der Arbeitsbedingungen zu investieren. Es wird die Bedürfnisse der Patienten nach einer evidence-basierten Behandlung, die Bedürfnisse der Mitarbeiter nach Zufriedenheit mit ihrer Arbeitssituation, nach Zumutbarkeit, Erträglichkeit und Ausführbarkeit der ihnen übertragenen Aufgaben sowie die Erwartungen der Krankenkassen und des Eigentümers nach Effizienzsteigerung nicht befriedigen können; der Bestand des Krankenhauses ist möglicherweise gefährdet.

Die dem Prinzip der sozialen Verantwortung verpflichtete Geschäftsführung begreift den Gewinn nicht als oberstes Unternehmensziel. Sie stellt die Gewinnerzielung in den Dienst der Befriedigung von Stakeholder-Anliegen und damit der langfristigen Sicherung des Krankenhauses.

\subsubsection{Begründungspflicht}

Das gelungene Management der sozialen Verantwortung zeichnet sich dadurch aus, dass unternehmerische Entscheidungen gegenüber den Betroffenen begründet werden und dass sie sich mittels guter Cründe verteidigen lassen (Steinmann; Löhr, S. 1of.). Dabei kann es sich bei den Gründen einerseits um materielle Normen handeln, also zum Beispiel um die Unternehmensziele und um die Anliegen, die von Stakeholdern geltend gemacht werden. Aber auch Verhaltens-Normen, die den Umgang zum Beispiel der Führungskräfte mit ihren Mitarbeitern, den Umgang der Führungskräfte und der Mitarbeiter mit Patienten oder das Verhalten der einschlägigen Krankenhaus-Instanzen zu Lieferanten steuern, zählen dazu. Es kann um prozessuale Normen gehen, also um Unternehmensstrukturen, die das Berücksichtigen der materiellen Normen bei unternehmerischen Ent- 
scheidungen fördern oder gar erst ermöglichen. Schließlich müssen sich die Entscheidungen durch rechtliche Normen begründen lassen.

\subsection{Die Rolle der Geschäftsführung}

Die Geschäftsführung prägt den Charakter eines Krankenhauses, und zwar nicht nur in ökonomischer, sondern auch in sozialer, kultureller und gesellschaftlicher Hinsicht. Die Mitglieder des Topmanagements beeinflussen das Verhalten der Führungskräfte und der Mitarbeiter in diesem Sinne kraft ihrer Persönlichkeit. Sie geben die Richtung und den Takt dadurch vor, dass sie weil sie ständig unter Beobachtung stehen - ihren Mitarbeitern Vorbild sind - in die eine oder andere Richtung.

So gesehen spielt die Geschäftsführung die zentrale Rolle hinsichtlich der Einführung und des dauerhaften Praktizierens des Managements der sozialen Verantwortung. Die Entwicklung, Implementierung und das dauerhafte Ermöglichen der neuen Unternehmensstrategie ist - und kann nur sein - „Chefsache“. Die Initiative für die Umstellung auf das bessere Management-Konzept geht von der Geschäftsführung aus. Sie stellt -im Sinne der Goldenen Regel - die als legitim akzeptierten Anliegen der diversen Stakeholder in den Mittelpunkt unternehmerischen Handelns. Sie begeistert Führungskräfte und Mitarbeiter, es ihr gleich zu tun.

Die Geschäftsführung gibt den Führungskräften
und Mitarbeitern die Möglichkeit zu entdecken,
welchen Vorteil es für alle Beteiligten hat, sozi-
al verantwortlich zu handeln. Sie bietet den zu-
nächst unentschlossenen Führungskräften und
Mitarbeitern an, sozial verantwortliches Handeln
im Krankenhaus-Alltag zu üben (in Anlehnung an
Minkmar, S. 23).

Die Entwicklung, die Implementierung und das dauerhafte Ermöglichen des Managements der sozialen Verantwortung ist ein radikaler Bruch mit den über eine lange Zeit hinweg eingeübten und praktizierten Verhaltensweisen. Sie können nur dann mit Erfolg realisiert werden, wenn die Geschäftsführung diese Umorientierung mittels der Instrumente des Managements der sozialen Verantwortung vorbereitet. Vor allem die einschlägigen Anliegen der von dem Strategiewech- sel betroffenen Führungskräfte und Mitarbeiter werden bei den anstehenden Entscheidungen berücksichtigt; die Entscheidungen werden auf der Basis einer argumentativen, dialogische Verständigung mit den Betroffenen vorbereitet und gefällt. Die Vorbereitung der Implementierung des Managements der sozialen Verantwortung wird somit zweifach genutzt: nämlich auch als Training für sozial verantwortliches Handeln im Routinebetrieb.

Das Topmanagement des Medizinbetriebes muss seine Mitarbeiter von der Notwendigkeit des Strategiewechsels und der Umgestaltung der Leitungsstruktur im Sinne der in Kapitel 6.2, insbesondere in den Kapiteln 6.2.4 bis 6.2.10 beschriebenen Anforderungen überzeugen. Diese Überzeugungsarbeit kann durch eine Vielzahl von Maßnahmen unterstützt werden (unter anderem Göbel, S. 272ff.):

1. Die Mitglieder der Geschäftsführung initiieren die Entwicklung/Weiterentwicklung des Unternehmens-Leitbildes im Sinne der Crundidee des Managements der sozialen Verantwortung. Sie nehmen sich die Zeit, um sich aktiv und umfassend an der Erarbeitung eines neuen Unternehmens-Leitbildes zu beteiligen.

2. Sie lassen einen konkreten Implementierungsplan erarbeiten und sorgen dafür, dass erste Umstellungsschritte möglichst rasch erfolgreich in die Tat umgesetzt werden. Die Mitarbeiter des Medizinbetriebes werden auf diese Weise am ehesten für die Umstellung gewonnen.

3. Sie praktizieren die im Unternehmens-Leitbild fixierten Führungsgrundsätze und geben damit ihren Mitarbeitern die Gelegenheit, an diesem Vorbild zu lernen.

4. Sie bringen ihre Bereitschaft und ihren Willen zur Realisierung der neuen Unternehmensstrategie dadurch zum Ausdruck, indem sie die als legitim akzeptierten Stakeholder-Anliegen bei unternehmerischen Entscheidungen explizit berücksichtigen, und dieses transparent machen und begründen.

5. Die Mitglieder der Geschäftsführung nutzen die ihnen zur Verfügung stehenden Medien - Besprechungen, Vollversammlungen, Empfänge, Interviews, Publikationen in Büchern, Fach- und/oder Hauszeitschriften und anderes -, um nach innen und außen für den Strategiewechsel zu werben. 


\subsection{Die Leitungsstruktur des Krankenhauses}

\subsubsection{Leitung und Verantwortung - künftige Entwicklungen}

Unter der Leitung einer Organisationseinheit versteht man

- das Vereinbaren von Zielen, die von dieser Organisationseinheit realisiert werden sollen, mit der vorgesetzten Führungskraft,

- die Übernahme der Verantwortung für die Durchführung der für die Zielerreichung notwendigen Maßnahmen, für das Erreichen der vereinbarten Ziele und für die daraus sich ergebenden Folgen,

- das Fällen der für das Erreichen der Ziele notwendigen Mittel-Entscheidungen und gegebenenfalls

- die Befugnis, das Ergebnis der Entscheidungen Dritten zur Ausführung anzuweisen.

Um einem Verantwortungsträger die Leitung einer klinischen Abteilung übertragen zu können, sind bestimmte Anforderungen zu erfüllen: Es sind dieses die Zuweisung eines eindeutig umrissenen Verantwortungsbereichs und eines mit diesem übereinstimmenden Entscheidungsspielraums. Es ist eine Leitungsstruktur erforderlich als Rahmen, der dem Verantwortungsträger die Chance bietet, das von ihm erwartete soziale Handeln innerhalb des ihm zur Verfügung stehenden Entscheidungsspielraums wahrzunehmen.

Die zunehmende Spezialisierung der in den Krankenhäusern tätigen Berufsgruppen - das gilt vor allem für die Ärzte - hat dazu geführt, dass Leistungsbereiche in Sub-Bereiche aufgeteilt werden. Die Zahl der Schnittstellen zwischen den an der Behandlung eines Patienten beteiligten Leistungs-Sub-Bereichen nimmt zu. Dieses hat Folgen für die Wahrnehmung von Verantwortung durch die in den Leistungs-Sub-Bereichen beschäftigten Verantwortungsträger. Der Behandlungsprozess wird von einer immer größer werdenden Zahl von Verantwortungsträgern beeinflusst; der Zusammenhang zwischen dem eigenen Tun und der Verwirklichung des Behandlungsziels wird immer mehr aufgelöst. Der einzelne Verantwortungsträger hat die Möglichkeit, sich von der ihm zugewiesenen Verantwortung zu entlasten (s. Beispiel). Es stellt sich daher die - im Rahmen dieses Kapitels zu beantwortende - Frage, wie die Leitungsstruktur des Krankenhauses beschaffen sein sollte, um dieses Defizit vermeiden zu können.

\section{Beispiel}

Das Ziel der medizinischen Leistungsbereiche eines Krankenhauses ist die Wiederherstellung der Gesundheit eines Patienten, die Linderung von Leiden und/oder das Leisten von Geburtshilfe. Die Verantwortung für das Einlösen dieses Zieles wird - bezogen auf einen einzelnen Patienten - einem dafür kompetenten Arzt zugewiesen. Dieser Arzt entscheidet, welche Maßnahmen der Diagnostik, der Therapie und der Behandlungspflege wann durchgeführt werden, damit der Patient so früh als möglich - aus medizinischer Sicht und unter Berücksichtigung der spezifischen Situation des Patienten (eventuell ist der Patient alt und/ oder lebt ohne Partner) - entlassen werden kann.

Je nach der Leitungsstruktur des Krankenhauses kann der Arzt die ihm zugewiesene Verantwortung nur eingeschränkt wahrnehmen. Wann nämlich die für das Erstellen der Diagnose erforderlichen Laboratoriums- und/oder Röntgenbefunde zur Verfügung stehen, wann der Patient gegebenenfalls operiert wird und wann die von dem Arzt angeordneten Maßnahmen der Behandlungspflege durchgeführt werden, entscheidet nicht der für den Patienten verantwortliche Arzt, sondern die für die genannten medizinischen Supportbereiche bzw. die für den Pflegebereich Verantwortlichen. Abweichungen von der von dem Arzt erarbeiteten Behandlungsplanung sind deshalb nicht ausgeschlossen.

Der Arzt hat somit auch nur einen eingeschränkten Einfluss darauf, ob das skizzierte Behandlungsziel in zeitlicher Hinsicht realisiert werden kann. Er wird - wenn das Behandlungsziel nicht realisiert wird - auf das Auseinanderfallen von Verantwortungsbereich und Entscheidungsspielraum verweisen und die Verantwortung für die Folgen für den Patienten ablehnen.

\subsubsection{Die Gestaltungsbereiche im Überblick}

Bei der weiteren Behandlung des Themas werden zwei Leitungsstruktur-Ebenen unterschieden (s. Abb. 35):

1. In der Mikro-Ebene steht die Beantwortung der Frage im Vordergrund, wie die Handhabung einzelner Teilfunktionen betrieblicher Leitungstätigkeit (s. Tab. 13) im Detail arrangiert werden muss, damit diese im Sinne des Managements der sozialen Verantwortung praktiziert werden können (s. Kap. 6.4.3). Dabei wird immer wieder danach gefragt, ob das Cestal- 
Tab. 13 Die Teilfunktionen betrieblicher Leitungstätigkeit

\begin{tabular}{ll} 
Teilfunktionen betrieblicher Leitungstätigkeit \\
Kernfunktionen & unterstützende Funktionen \\
\hline Zielsetzung & Motivation \\
\hline Planung & Repräsentation \\
\hline
\end{tabular}

Organisation

Realisation

Kontrolle

tungsergebnis den Grundpostulaten des Managements der sozialen Verantwortung entspricht (s. Beispiel 1).

\section{Beispiel 1}

Die Planung - zum Beispiel der Leistungen und/oder der Kosten - wird so organisiert, dass die von der Planung betroffenen entweder persönlich oder vertreten durch einen Agenten an den Planungsgesprächen und -entscheidungen teilnehmen und ihre Interessen vertreten können. Eine dialogische Verständigung mit dem Planungsträger unter anderem über die Planungsmethoden, über die Faktoren, die das Ergebnis der Planung beeinflussen, und über das Planungsergebnis wird ermöglicht (s. unter anderem die Grundpostulate „Berücksichtigung der Interessen Betroffener“, „Förderung der argumentativen, dialogischen Verständigung“ und „Förderung der Mündigkeit der Stakeholder").

Bezogen auf das Beispiel in Kapitel 6.4.1 ist deshalb zu fordern, dass der für den Patienten verantwortliche Arzt die Behandlungsplanung in Abstimmung mit den Supportbereichen und dem Pflegebereich erarbeitet.

2. In der Makro-Ebene wird die Organisation der Leitungstätigkeit dargestellt (s. Kap. 6.4.4). Sie ist der Rahmen für die Realisierung der Managementfunktionen und für die Zuweisung von Verantwortung zu einem Verantwortungsträger. In diesem Zusammenhang ist zu überlegen, wie die aufbauorganisatorische Grundstruktur des Krankenhauses gestaltet sein muss. Es stellt sich die Frage, in wie viele Leitungsebenen ein Unternehmen vernünftigerweise gegliedert wird; es fragt sich, ob die Leitungstätigkeit weitgehend an die Basis des Unternehmens verlagert oder in dessen Spitze zentral wahrgenommen wird. Schließlich stellt sich die Frage nach der personellen Be- setzung der Instanzen, denen die Wahrnehmung der Managementfunktionen übertragen wird, und wie - wenn es mehrere Personen sind - die Zusammenarbeit dieser Personen erfolgt.

\section{Beispiel 2}

Die Instanzen, die die Teilfunktionen betrieblicher Leitungstätigkeit wahrnehmen, sind unterschiedlich organisiert. So gibt es Krankenhäuser mit einer Ein-PersonenGeschäftsführung. Andere Krankenhäuser und/oder Leistungsbereiche in diesen werden von einem Kollegialorgan geleitet, besetzt im Regelfall mit einem Arzt, einer Krankenpflegekraft und einem Kaufmann. Ist Letzteres der Fall, bedarf es der Festlegung, welche Aufgaben von welchem Mitglied in eigener Verantwortung und welche Aufgaben von den Mitgliedern des Kollegialorgans gemeinsam wahrgenommen werden und wie für diesen Fall bei konträren Auffassungen unter den Mitgliedern eine Entscheidung herbeigeführt wird (s. unter anderem die Grundpostulate „Berücksichtigung der Interessen Betroffener“, „Förderung der argumentativen, dialogischen Verständigung“ und „Förderung der Mündigkeit der Stakeholder“).

Bezogen auf das Beispiel in Kapitel 6.4.1 wird deshalb von den Ärzten immer wieder gefordert, dass sie gegenüber den für die Behandlungspflege zuständigen Krankenpflegekräften unmittelbar weisungsbefugt sind, damit sie Verantwortung für das Realisieren eines Behandlungszieles uneingeschränkt wahrnehmen können.

\subsubsection{Die Teilfunktionen betrieblicher Leitungstätigkeit}

\section{Die Teilfunktionen im Überblick}

In der betriebswirtschaftlichen Literatur und in der Unternehmens-Praxis wird der Prozess des Managens eines Unternehmens unterschiedlich strukturiert. Dieses betrifft vor allem die Abgrenzung zwischen der Zielsetzung, der Planung und der Entscheidungstätigkeit. Als Grundlage für die Darstellung der Voraussetzungen für sozial verantwortliches Handeln im Krankenhaus wird die in Tabelle 13 ausgewiesene Gliederung der Leitungstätigkeit in Teilfunktionen betrieblicher Leitungstätigkeit verwendet. Die Beschreibung des strategischen Managementprozesses in Kapitel 3.1 ist der unten folgenden inhaltlich weitgehend identisch, wenngleich terminologisch einige Unterschiede gegeben sind. In Kapitel 3.1 wird der Bezug zu dem Thema „sozial verantwortliches 
Handeln“ unmittelbar hergestellt, während in dem vorliegenden Abschnitt zunächst eine allgemeine Beschreibung der Funktionen erfolgt und erst in den folgenden Abschnitten die Anforderungen dargestellt werden, die berücksichtigt werden müssen, damit sozial verantwortliches Handeln nicht behindert wird.

Die Entscheidungstätigkeit, die in Kapitel 6.5 thematisiert wird, wird in dieser Darstellung nicht als gesonderte Leitungstätigkeit ausgewiesen (anders z. B. bei Schierenbeck, S. 97). Begründet wird dieses damit, dass jede Teilfunktion betrieblicher Leitungstätigkeit Entscheidungen verlangt.

- Wenn die Mission, die Unternehmensgrundsätze und die Vision, also das normative Grundgerüst des Krankenhauses festgelegt worden ist, beginnt der Prozess der Aufgabenerfüllung mit der Entwicklung und Verabschiedung der Unternehmensziele (Zielsetzung). Das Zielsystem des Krankenhauses ist das Ergebnis einer strategischen Umwelt- und Unternehmensanalyse. Es geht auf dessen institutionellen Sinn zurück - warum wird ein Krankenhaus gegründet und betrieben? -; es schließt die Sicherung der Existenz des Krankenhauses und der darin vorgehaltenen Arbeitsplätze (= Formalziel) als Basis für die Deckung des Bedarfs an medizinischen Leistungen (= Sachziel) ein (Eichhorn, S. 97ff.).

- Während die Zielsetzung das künftige Verhalten des Unternehmens determiniert, ist es Aufgabe der Planung, die Zielsetzung unter Berücksichtigung der zum Zeitpunkt der Zielrealisierung gegebenen internen und externen Umsetzungsbedingungen in operationale Vorgaben zu transformieren.

Damit hat die Planung zwei Funktionen (Hellfors, S. 41): Mit der zukunftserkennenden Funktion stellt sie zukünftige Ereignisse und Entwicklungen fest, sucht also die Antwort zum Beispiel auf die Frage, mit wie viel Patienten die klinische Abteilung $\mathrm{X}$ im nächsten Planungsjahr wahrscheinlich rechnen kann. Mit der zukunftsgestaltenden Funktion legt die Planung - z.B. im Rahmen einer Zielvereinbarung zwischen der Geschäftsführung und dem für die Leitung der klinischen $\mathrm{Ab}$ teilung X Verantwortlichen - die Anzahl der durch diese Abteilung im nächsten Planungsjahr zu behandelnden Patienten fest und die Ressourcen (Mitarbeiter, Materialien, Geräte usw.), die für die Realisierung der geplanten
Leistung erforderlich sind. Sie stellt damit in Form von strategischen Programmen die Verbindung zu der operativen Planung her.

- Es folgen die Gestaltung des betrieblichen Kombinationsprozesses (Organisation), also die Festlegung der Leitungsstruktur des Krankenhauses und der Arbeitsabläufe in diesem als Basis für die Realisierung des Planungsergebnisses sowie

- der Vollzug der Maßnahmen (Realisation).

- Dessen Ergebnis wird durch einen Vergleich mit einer Norm - dem durch die Aufgabenerfüllung zu erreichenden Zustand einschließlich der Berücksichtigung von Stakeholder-Bedürfnissen - kontrolliert (Kontrolle), um bei Abweichungen durch steuernde Eingriffe in die Realisierung eine möglichst große Annäherung zwischen dem tatsächlich Erreichten und der Zielsetzung gewährleisten zu können. $\mathrm{Zu}$ der Analyse eventuell festgestellter Abweichungen zwischen der Norm und dem tatsächlich Erreichten gehört es auch, zu prüfen, ob das gesetzte Ziel und/oder das Ergebnis der Planung überhaupt erreichbar gewesen sind und/oder ob die gewählte Organisation zur Zielerreichung geeignet war. Gegebenenfalls erstrecken sich die steuernden Eingriffe auch auf die Revision der Zielsetzung und/oder die der Planung und/oder die der Organisation.

- Ob und inwieweit das gesteckte Ziel erreicht wird, hängt auch von der Übereinstimmung zwischen dem von den Mitarbeitern aufgrund ihrer Rolle erwarteten und deren tatsächlichen Verhalten ab. Eine eventuell gegebene Diskrepanz, gegebenenfalls auch in Hinblick auf sozial verantwortliches Handeln, wird durch spezifische Führungsleistungen ausgeglichen (Motivation) (Staehle 1999, S. zooff. und 3olff.).

- Zu den Teilfunktionen betrieblicher Leitungstätigkeit zählt schließlich die Vertretung der Interessen des eigenen Aufgabenbereiches gegenüber gleich- oder übergeordneten Stellen (Repräsentation). Durch die Einflussnahme auf Entscheidungen gleich- oder übergeordneter Stellen wird versucht, die Voraussetzungen für die Aufgabenerfüllung des eigenen Aufgabenbereiches möglichst vorteilhaft zu gestalten.

Die Berücksichtigung von Stakeholder-Anliegen ist hinsichtlich vor allem dreier Aspekte der Willensbildung und -durchsetzung von Relevanz. 
Sie sind Gegenstände der nun folgenden Betrachtung. Dabei handelt es sich um

1. die Handhabung der beiden Teilfunktionen betrieblicher Leitungstätigkeit Planung und Kontrolle und deren Zusammenfassung in einer einheitlichen Teilfunktion Controlling

2. einige Elemente der Organisation des Krankenhauses; dabei wird die Organisation im institutionellen Sinne als die Ordnung, also als eine Summe von Regeln verstanden, die für das zielgerichtete Zusammenwirken einer Vielzahl von Aufgabenträgern in einem multipersonal und arbeitsteilig organisierten Unternehmen erforderlich ist und um

3. diverse Felder des Personalmanagements, dessen Aufgabe es ist, die personellen Voraussetzungen für die Realisierung der unter Ziffer 2. genannten Ziele zu schaffen.

Die Teilfunktion „Zielsetzung“ fehlt in dieser Aufstellung. Der Grund: In der hier verwendeten Begriffsbestimmung wird unter Zielsetzung ein Vorgang verstanden, der das Sachziel und das Formalziel des Krankenhauses festlegt und somit im Regelfall als Datum für das Handeln der Geschäftsführung angesehen werden kann. So wie der Begriff „Zielsetzung“ in der Praxis häufig verwendet wird, ist er im Rahmen der hier verwendeten Systematik der Teilfunktionen betrieblicher Leitungstätigkeit mit der zukunftsgestaltenden Funktion der Planung weitgehend identisch.

Die übrigen Teilfunktionen betrieblicher Leitungstätigkeit werden - soweit sie für die Umorientierung des Managens eines Krankenhauses relevant sind - in Kapitel 8 zum Thema gemacht.

Die Darstellung der Teilfunktionen betrieblicher Leitungstätigkeit beginnt jeweils mit einer kurzen Charakterisierung der Teilfunktion und der Beantwortung der Frage, welche Ziele mit deren Hilfe verfolgt werden und welche Wirkungen von deren Realisierung ausgehen. Danach werden die Anforderungen an den Prozess der Gestaltung der Teilfunktion (Controlling und Personalmanagement) bzw. an deren Ergebnis (Organisation) beschrieben. Es wird gezeigt, durch welche Art von Prägung das Wahrnehmen sozialer Verantwortung durch die Geschäftsführung, die Führungskräfte und die Mitarbeiter ermöglicht und unterstützt werden kann.

\section{Controlling}

\section{a) Charakterisierung}

Die Komplexität der Behandlungs-, Support- und Betriebsführungsprozesse nimmt zu. Die Ursachen dafür sind unter anderem die Herausforderungen, mit denen sich die Geschäftsführung konfrontiert sieht. In deren Folge werden Veränderungen technischer, organisatorischer, personeller und finanzieller Art notwendig, um in dem zunehmenden Wettbewerb auf dem Absatz- und auf dem Arbeitsmarkt bestehen zu können.

In immer größerem Maße werden Entscheidungen auf der Basis unsicherer und unvollständiger Informationen getroffen. Das Risiko, dass das Ergebnis eines durch eine Entscheidung ausgelösten Prozesses nicht den Erwartungen des Entscheidungsträgers entspricht, wird größer. Dieses gilt insbesondere für Planungen zur langfristigen Existenzsicherung des Krankenhauses.

Das Risiko, dass durch nachgeordnete Instanzen Entscheidungen gefällt werden, deren Ergebnis mit den Intentionen der Geschäftsführung nicht übereinstimmt, nimmt mit zunehmender Dezentralisierung der Leitungstätigkeit, mit der Erweiterung der Delegation von Entscheidungsbefugnissen von einem Vorgesetzten an seine Mitarbeiter und der Spielräume, die den Entscheidungsträgern eingeräumt werden, zu. Hinzu kommt, dass die Übersetzung des Unternehmenszieles in konkretes betriebliches Handeln ein Prozess ist, dessen Ergebnis durch Entscheidungen in einer Vielzahl von Subsystemen beeinflusst wird (Schreyögg 1991, S. 264f.) (s. Beispiel).

\section{Beispiel}

Eine neue Behandlungsmethode, die als Ergebnis einer unternehmerischen Entscheidung der Geschäftsführung entwickelt worden ist, kann möglicherweise nicht wie geplant eingeführt werden, weil das Subsystem „Personalabteilung" die Mitarbeiter mit der dafür notwendigen Qualifikation nicht (rechtzeitig) zur Verfügung stellen kann.

Um auch unter diesen Bedingungen eine hohe Qualität und Effizienz der Patientenbehandlung gewährleisten zu können, bedienen sich die Geschäftsführung und die für die Leistungsbereiches des Krankenhauses Verantwortlichen als Entscheidungs- und Steuerungshilfe des Controllings, indem 
- die Teilfunktionen betrieblicher Leitungstätigkeit „Planung“ und „Kontrolle“ zu einem einheitlichen Management-Instrument „Controlling“" zusammengefasst werden,

- die Teilfunktion Controlling das Management mit entscheidungsrelevanten Informationen versorgt und im Sinne der Beratung die von dem Management zu fällenden Entscheidungen und deren Umsetzung vorbereitet und

- das Management bei der Korrektur eventuell aufgetretener Fehlentwicklungen unterstützt.

So gesehen ist Controlling eine der zentralen Voraussetzungen dafür, um einem Verantwortungsträger, der die Folgen seiner Entscheidungen mit ausreichender Sicherheit nicht vorhersehen kann, Verantwortung zuweisen zu können. Mit Hilfe des Controllings werden Unsicherheiten abgebaut und damit sozial verantwortliches Handeln erst ermöglicht. Der Gestaltung des Controllings kommt deshalb für das Wirksamwerden der Grundideen des Managements der sozialen Verantwortung eine besondere Bedeutung zu.

Die bisher skizzierte - gewissermaßen gewohnte - Charakterisierung der Managementfunktion ordnet dem Controlling eine Führungskoordinierungsfunktion und eine Führungsunterstützungsfunktion zu. Wenn das Controlling in den Dienst das Management der sozialen Verantwortung gestellt wird, übernimmt es darüber hinaus die Funktion einer „Metaführung“ (Göbel 2006,
S. 245; Naegler et al., S. 249f.). Es hinterfragt in dieser Rolle das Handhaben des Managements der sozialen Verantwortung; es leistet einen Beitrag dafür, dass eine verantwortungsbewusste Unternehmensführung dauerhaft wirksam werden kann.

\section{b) Anforderungen an die Gestaltung des Controllings} So gesehen lassen sich für die Gestaltung des Controllings sowie für die der Planung und der Kontrolle die im Folgenden genannten GestaltungsFelder und -Anforderungen identifizieren. Die Gestaltungs-Felder werden der besseren Übersicht wegen zu drei Gestaltungs-Feld-Gruppen zusammengefasst (s. Tab. 14).

\section{Anforderungen an das Controlling}

Gestaltungs-Feld-Gruppen; Gestaltungs-Feld; Anforderungen an die Gestaltung

\section{Kulturelle und strategische Aspekte des Controllings}

\section{Controlling-Leitbild}

Die Einführung des Controllings (was heutzutage nur noch selten notwendig wird) bzw. dessen Ausbau wird bei den Führungskräften und den Mitarbeitern skeptisch beurteilt. Wenn von der Geschäftsführung nicht anders kommuniziert, sehen die Führungskräfte und die Mitarbeiter in der Einführung/der Erweiterung des Controllings nicht selten ausschließlich den Versuch des Top-Managements, die Effizienz der Patientenbehandlung zu steigern, und zwar zu ihren und der Patienten Lasten.

\section{Tab. 14 Gestaltungs-Feld-Gruppen des Controllings}

\begin{tabular}{|c|c|}
\hline Gestaltungs-Feld-Gruppen & Gestaltungs-Feld \\
\hline $\begin{array}{l}1 \text { Kulturelle und strategische Aspekte } \\
\text { des Controllings }\end{array}$ & $\begin{array}{l}\text { Controlling-Leitbild mit unter anderem den Inhalten } \\
\text { Zielsetzung des Controllings } \\
\text { Bedeutung des Controllings für die Geschäftsführung } \\
\text { Umgang mit Fehlern } \\
\text { Personelle Voraussetzungen }\end{array}$ \\
\hline 2 Organisation des Controllings & $\begin{array}{l}\text { Gegenstände der Planung/der Kontrolle* } \\
\text { Eingliederung des Controllings in die Krankenhausorganisation } \\
\text { Berichtswesen }\end{array}$ \\
\hline 3 Prozess des Controllings & $\begin{array}{l}\text { Prozess der Planung } \\
\text { Kontrollprozess }\end{array}$ \\
\hline
\end{tabular}

* Die Gegenstände der Planung sind immer auch Gegenstände der Kontrolle. Deshalb werden als Gegenstände der Kontrolle nur jene gesondert genannt, die nicht auch Planungsgegenstände sind. 
Es empfiehlt sich deshalb, die Einführung/die Erweiterung des Controllings mit der Erarbeitung eines Controlling-Leitbildes vorzubereiten. Gegenstände dieses Leitbildes sollten insbesondere sein:

- die Zielsetzung des Controllings,

- die Bedeutung des Controllings für die Geschäftsführung und

- der Umgang mit Fehlern.

Die Erarbeitung des Controlling-Leitbildes wird nicht nur initiiert von der Geschäftsführung. Von zentraler Bedeutung für die Akzeptanz des Controllings ist es, dass sich die Geschäftsführung die Zeit nimmt, um sich an der Entwicklung des Controlling-Leitbildes im Sinne einer vertrauensbildenden Maßnahme aktiv und gemeinsam mit den Führungskräften und den Mitarbeitern zu beteiligen und dass auf diese Weise die Anliegen der Führungskräfte und der Mitarbeiter in die Gestaltung des Controllings einbezogen werden. Das Controlling-Leitbild ist das Ergebnis einer argumentativen, dialogischen Verständigung zwischen der Geschäftsführung und den Führungskräften und Mitarbeitern.

Die Geschäftsführung gestaltet den Prozess der Leitbildentwicklung so, dass sich die Führungskräfte und die Mitarbeiter als mündige Gesprächspartner angenommen wahrnehmen.

Mit dem Erarbeiten des Controlling-Leitbildes kommt die Geschäftsführung ihrer Verpflichtung nach, zu begründen, welche Ziele mit dem Controlling verfolgt werden, welche Bedeutung das Controlling für die Verantwortungsträger hat und wie künftig mit Fehlern umgegangen werden soll.

\section{Zielsetzung des Controllings}

Vor allem muss deutlich werden, dass

- mit der Einführung des Controllings dazu beigetragen wird, eine Organisation zu entwickeln, die es den Führungskräften und den Mitarbeitern ermöglicht, ihre Ziele als Agenten der Patienten verwirklichen zu können, also ihre Fähigkeiten und Fertigkeiten in vollem Umfang für die Befriedigung der Patienten-Bedürfnisse wirksam werden zu lassen (Straub, S. 371),

- ein hinreichend großer Nutzen generiert werden kann, der möglichst vielen Stakeholdern, vor allem auch den Patienten und den Mitarbeitern, zu Gute kommt und der den Stakeholdern zeigt, dass der organisatorische und technische Aufwand sowie der Aufwand für notwendige Änderungen des Verhaltens der Führungskräfte und der Mitarbeiter gerechtfertigt sind und dass

- mit der Einführung des Controllings dazu beigetragen wird, dass das Krankenhaus seiner Gemeinwohlverpflichtung nachkommen kann.

In der Zielsetzung des Controllings muss im Sinne der MetaFührung durch das Controlling manifest werden, dass

- zu dessen Gegenständen die Realisierung des Managements der sozialen Verantwortung gehört.

\section{Bedeutung des Controllings für die Geschäftsführung}

Die Einführung bzw. die Weiterentwicklung des Controllings ist nicht frei von Risiken. Eines dieser Risiken ist die Sorge vieler Führungskräfte und Mitarbeiter vor der Einschränkung ihres Entscheidungsspielraumes bzw. vor dem Aufbau organisationaler Barrieren, die das Wahrnehmen von Verantwortung behindern. Sie befürchten Machtverlust. Sie sind deshalb für die Entwicklung, die Implementation und die laufende Realisierung des Controlling-Konzepts häufig nicht ausreichend motiviert.

Die Geschäftsführung ist deshalb aufgerufen, sich mit den Führungskräften sowie den Mitarbeitern darauf zu verständigen, welche Art von Bedeutung das Controlling haben wird. Soll das Controlling

- ein Kontroll-/Planungs-Instrument nur für die oberste Leitungsebene oder

- eine Informationsbörse für alle Führungskräfte und Mitarbeiter oder zusätzlich auch

- eine Informationsbörse für alle Stakeholder sein?

Im Sinne der Grundidee des Managements der sozialen Verantwortung ist zu fordern, dass das Controlling als eine möglichst umfassende Informationsbörse ausgestaltet wird (Einschränkungen unter anderem mit Blick auf die strategische Unternehmensplanung und auf personelle Angelegenheiten nicht ausgeschlossen). Dann nämlich wird deutlich werden, welche Stakeholder-Interessen bei unternehmerischen Entscheidungen berücksichtigt worden sind; das Krankenhaus kommt seiner Pflicht zur Begründung seiner Entscheidungen nach. Es leistet einen Beitrag zur argumentativen, dialogischen Verständigung und zu Förderung der Mündigkeit der Stakeholder.

\section{Umgang mit Fehlern}

Es ist zu fordern, dass die durch das Controlling festgestellten Fehlentwicklungen nicht nur personalisiert und sanktioniert werden, sondern dass diese zu allererst als Chance für das künftige Vermeiden von Fehlern, als Anlass zur Verbesserung der Struktur des Krankenhauses und der Behandlungs- und Serviceprozesse verstanden werden. Nur so kann ein Beitrag zur Förderung der Stakeholder-Mündigkeit geleistet und die Bereitschaft zur Offenlegung von Fehlern gefördert werden. Nur dann, wenn die Mitarbeiter, aber auch andere Stakeholder die Gewissheit haben, dass Offenheit nicht schadet, dass Willkür ausgeschlossen ist, wird sich die dafür notwendige Transparenz herstellen lassen (Hildebrand 2001, S. 338).

\section{Personelle Voraussetzungen}

Insbesondere hinsichtlich der Kontrolle der Aufgabendurchführungs-Prämissen (Zielsetzung, Planung und Organisation) gilt es, die Voraussetzungen für einen verantwortungsvollen und souveränen Umgang mit dem Instru- 
ment Controlling zu schaffen. Wichtig ist vor allem, dass die für die Realisierung der Teilfunktionen betrieblicher Leitungstätigkeit Zielsetzung, Planung und Organisation Verantwortlichen offen sind für eine dialogorientierte Auseinandersetzung mit den Ergebnissen der Kontrolle.

Die Wirksamkeit der Kontrolle vor allem der Aufgabendurchführungs-Prämissen setzt einen souveränen, mündigen Controller voraus und ein Betriebsklima, in dem der Controller seine Rolle als kritischer "Counterpart" der Verantwortlichen (Weibler; Lucht, S. 886) - gegebenenfalls unterstützt durch die Geschäftsführung - auch über mehrere Hierarchieebenen hinweg wahrnehmen kann.

\section{Organisation des Controllings}

\section{Gegenstände der Planung/der Kontrolle}

Die Planung geht herkömmlicherweise der Frage nach, welche Leistungen wann erbracht und welche Ressourcen dafür benötigt werden und zur Verfügung gestellt werden sollen. In der Krankenhauspraxis spielt bei diesen Überlegungen die ökonomische Vernünftigkeit des Ressourcen-Einsatzes eine zentrale und bisher noch nahezu ausschließliche Rolle.

Diese Tendenz wird dadurch verstärkt, dass staatliche Planung durch Wettbewerb als Ordnungsidee zur Sicherstellung eines ausreichend hohen Angebotes an medizinischen Leistungen abgelöst wird. Es kann nicht mehr aus- geschlossen werden, dass die formalen Hürden, die derzeit einen Preiswettbewerb in nur sehr engen Grenzen zulassen, überwunden werden, und der finanzielle Rahmen für die Finanzierung der Betriebs- und der Investitionskosten immer enger gesteckt wird. Deshalb muss damit gerechnet werden, dass Entscheidungen im Krankenhaus auf allen seinen Ebenen ausschließlich auf der Grundlage ökonomischer Vorteilhaftigkeit im Interesse des Krankenhauses gefällt werden. Negative Auswirkungen auf die von den Entscheidungen Betroffenen können die Folge sein. Es ist deshalb zu fordern, dass die Bereiche und die Gegenstände des Controllings entsprechend dem Stakeholder-Konzept und der daraus resultierenden Erweiterung der Balanced Scorecard um eine Soziale Perspektive (Greulich et al., S. 70) (s. Abb. 39) erweitert werden, dass das Controlling nicht nur das Erreichen der Ziele des Krankenhauses in seine Arbeit einbezieht.

Wenn die Planung in den Dienst des Managements der sozialen Verantwortung gestellt wird, wenn also die Bedürfnisse diverser Betroffener berücksichtigt werden sollen und wenn dem Grundpostulat „Beschränkung des Gewinnprinzips" angemessen Geltung verschafft werden soll, dann bedarf es auch deshalb einer Erweiterung des Katalogs der Planungsgegenstände im Sinne einer erweiterten Balanced Scorecard (s. Abb. 39). Nicht nur die Planungsgegenstände aus der finanziellen Perspektive, der Prozess- und der Kunden-Perspektive sowie der Perspektive von Lernen und Innovation, sondern vor allem auch die der

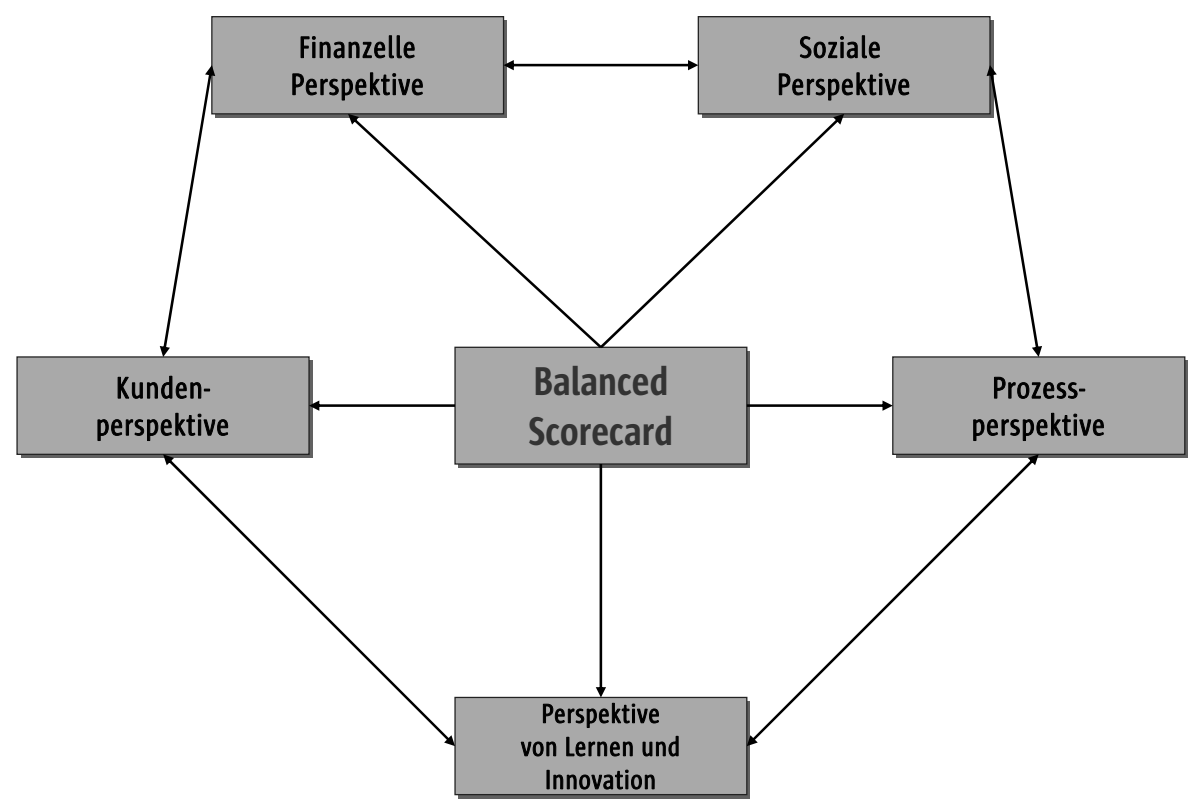

Abb. 39 Krankenhaus-spezifische Balanced Scorecard 
sozialen Perspektive werden in die Planung einbezogen. Möglicherweise werden sonst nur die Handlungen gefördert, die kurzfristig quantitativ messbare Ergebnisse bringen. Die Maßnahmen dagegen, deren Ergebnisse qualitativer Art sind und nur mit beträchtlichem Aufwand messbar gemacht werden können und erst mittel- und langfristig zu den gewünschten Ergebnissen führen - wie z.B. zu der Akzeptanz des Krankenhauses durch die Menschen in seinem Einzugsbereich -, kommen zu kurz. Dabei handelt es sich aber gerade bei diesen Größen um jene, die die Basis für die langfristige Sicherung des Krankenhauses sind.

So gesehen zählen zu den Gegenständen der Planung beispielsweise jene Maßnahmen, die der kontinuierlichen Verbesserung der Behandlungsqualität sowie der Arbeitsbedingungen und damit der Steigerung der Patientenund Mitarbeiterzufriedenheit dienen. Geplant werden zum Beispiel auch jene Maßnahmen, die im Sinne der Gemeinwohlverpflichtung des Krankenhauses ein bedarfsgerechtes Angebot an medizinischen Leistungen und die uneingeschränkte Erreichbarkeit des Krankenhauses gewährleisten. Damit wird dem Bedürfnis sowohl der Menschen, die im Einzugsbereich des Krankenhauses leben, als auch den diese gegebenenfalls einweisenden Ärzten entsprochen.

Gewissermaßen als Basis der "Metaführung" werden die Entwicklung, die Implementation und die dauerhafte Ermöglichung des Managements der sozialen Verantwortung geplant und kontrolliert (Wittmann, S. 124).

Zu den Gegenständen der Kontrolle gehören außer den schon genannten Gegenständen der Planung auch die Prämissen der Aufgabendurchführung, nämlich die Planung und die Organisation.

\section{Eingliederung des Controllings}

in die Krankenhausorganisation

Ein sozialorientiertes Führungskonzept zeichnet sich dadurch aus, dass Planungs- und Handlungsautorität und Verantwortung zusammengefasst sind. Ein solches Führungskonzept sieht vor, dass

- Planung je nach der Bedeutung der Planungsinhalte für die Unternehmensentwicklung und je nach ihrem zeitlichen Horizont entweder als strategische Planung in der obersten Leitungsebene oder als operative Planung möglichst dezentral, das heißt am Ort der operativen Verantwortung, und dass

- Kontrolle in erster Linie als Selbstkontrolle durch den jeweils Verantwortlichen

realisiert werden. So gesehen wird Controlling als strategisches Controlling in der obersten und als operatives Controlling eher an der Basis der Unternehmensorganisation wahrgenommen.

Mit dieser Art von Controlling-Organisation fördert die Geschäftsführung die Mündigkeit der Führungskräfte und der Mitarbeiter. Deren Bedürfnis nach Sicherheit - im Sinne von Bewältigenkönnen der ihnen übertragenen Aufgaben -, nach Wertschätzung und nach Selbstverwirklichung wird befriedigt.

\section{Berichtswesen}

Eine dem Management der sozialen Verantwortung verpflichtete Unternehmensführung löst einen spezifischen Informationsbedarf aus, der vor allem auch von dem Informationsbedarf externer Empfänger geprägt wird und der mit den üblichen betrieblichen Kennzahlen nicht befriedigt werden kann. In Sozialbilanzen werden die gesellschaftlichen und die betrieblichen Auswirkungen der Tätigkeit des Krankenhauses dargestellt (Göbel 2006, S. 247; Eisele, S 494ff.; Pfaff, S. 11ff.). Qualitätsberichte mit der Präsentation der Krankenhaus-Leistung in qualitativer und in quantitativer Hinsicht, aber auch die umfassende Darstellung der Leistungen des Personalmanagements sind Elemente von Sozialbilanzen, die der Öffentlichkeit zugänglich gemacht werden - so z.B. in dem Geschäftsbericht 2008 und dem Medizinischen Jahresbericht 2006/2007 der Helios Kliniken GmbH und in dem Qualitätsbericht 2007 des Wiener Krankenanstaltenverbundes.

Anhand dieser Dokumente können die diversen Stakeholder feststellen, ob überhaupt und in welchem Ausmaß ihren Anliegen bei unternehmerischen Entscheidungen entsprochen worden ist. Das Krankenhaus kommt seiner Pflicht zur Begründung unternehmerischer Entscheidungen nach. Es leistet einen Beitrag zur argumentativen, dialogischen Verständigung zwischen den Verantwortungsträgern des Krankenhauses und den Stakeholdern und zu Förderung der Mündigkeit der Stakeholder.

\section{Prozess des Controllings}

\section{Prozess der Planung}

Es sollte versucht werden, die von den Auswirkungen unternehmerischer Planung Betroffenen möglichst persönlich an der Planung zu beteiligen, damit diese ihre Anliegen unmittelbar und ungefiltert in den Planungsprozess einbringen können.

Die persönliche Beteiligung ist allerdings nicht immer möglich:

- Die Betroffenen sind zum Beispiel wegen Krankheit oder wegen Unabkömmlichkeit an einem anderen Ort nicht im Stande, ihre Interessen persönlich wahrzunehmen.

- Die Zahl der Betroffenen ist so groß, dass die Geschäftsführung nicht mit allen persönlich verhandeln kann.

- Die Anliegen der Betroffenen sind so unterschiedlich, dass es vor Beginn der Verhandlungen mit der Geschäftsführung eines Ausgleichs der Interessen innerhalb der Gruppe der Betroffenen bedarf. Wenn dieses 
nicht gelingt, nutzt auch die persönliche Beteiligung an den Planungsgesprächen nichts.

Nicht selten lassen sich die Betroffenen durch Agenten vertreten. Im Krankenhaus sind dieses zum Beispiel die Mitarbeiter-Vertretung, ein Ethikkomitee und/oder eine $0 \mathrm{~m}$ budsperson (s. Kap. 7.4.2), die die Interessen der Mitarbeiter, der Patienten, der Bevölkerung im Einzugsbereich des Krankenhauses und die der niedergelassenen Ärzte bei der Planung wahrnehmen.

Damit sich die Betroffenen oder deren Agenten kompetent an der Planung beteiligen können und damit ein Dialog als Grundlage für eine Verständigung hinsichtlich des Planungsergebnisses wirklich zustande kommen kann, muss verlangt werden, dass alle an dem Planungsprozess beteiligten möglichst frühzeitig mit den erforderlichen Materialien versorgt werden.

Die Beteiligung der Betroffenen an der Planung hat dagegen nicht selten eine Alibifunktion - nämlich dann, wenn den Beteiligten der fertige Plan zur Begutachtung oder Stellungnahme und zur Zustimmung vorgelegt wird; so sehen z.B. das Aktienrecht und die Personalvertretungsgesetze vor, dass dem Aufsichtsrat oder der Personalvertretung der fertige Plan, versehen mit einer ausführlichen Begründung, zur Zustimmung vorgelegt wird. Die Möglichkeiten zur Einflussnahme auf das Ergebnis des Planungsprozesses sind damit - inhaltlich und zeitlich gesehen - im Regelfall eher begrenzt. Es handelt sich bei dieser Vorgehensweise nicht um die Beteiligung der Betroffenen an dem Planungsprozess im Sinne einer argumentativen, dialogischen Verständigung, sondern um die Aufforderung an die Betroffenen, bereits gefällte Entscheidungen zu kontrollieren (Wittmann, S. 125). Diese Vorgehensweise steht nicht nur im Widerspruch zu dem Grundpostulat „Förderung der argumentativen, dialogischen Verständigung“, sondern auch zu dem Grundpostulat „Berücksichtigung der Interessen Betroffener". Sie sollte deshalb, obwohl durch einschlägige Gesetze gedeckt und obwohl in der Praxis nicht immer leicht zu realisieren, im Sinne der Grundideen des Managements der sozialen Verantwortung korrigiert werden.

Das Management der sozialen Verantwortung fordert, dass Planungsautorität und Handlungsautorität für jeweils einen Verantwortungsbereich zusammengefasst werden. Die Planung sollte am Ort der Verantwortung institutionalisiert werden. Das schließt nicht aus, dass im Sinne des Gegenstromverfahrens der Planungsprozess mit der ausführlich begründeten Vorgabe der durch die Geschäftsführung für alle Bereiche des Unternehmens festgelegten globalen Unternehmensziele gestartet wird. Die daraufhin von den für die Leistungsbereiche Verantwortlichen vorgelegten Teilpläne werden sodann von dem Controlling auf ihre Eignung für das Erreichen der Unternehmensziele und auf ihre Verträglichkeit mit anderen Teilplänen hin evaluiert. Erst danach werden sie auf der Grundlage einer erneuten argumentativen, dialogischen Verständigung als Zielvereinbarung zwischen der Geschäftsführung und den Verantwortlichen und damit als Handlungsgrundlage verabschiedet. Das Bedürfnis der Führungskräfte und der Mitarbeiter nach Berücksichtigung diverser ihrer Bedürfnisse, so zum Beispiel nach Sicherheit - im Sinne von Bewältigenkönnen der übertragenen Aufgaben -, nach Wertschätzung und nach Selbstverwirklichung wird damit befriedigt. Die Führungskräfte und Mitarbeiter werden als mündige Stakeholder wahrgenommen.

Planung ist in vielen Unternehmen inhaltlich eindeutig strukturiert und in zeitlicher Hinsicht aus guten Gründen streng reglementiert. Das verhindert aber nicht selten, dass relevante Gegebenheiten nicht rechtzeitig und nicht vollständig erkannt und deshalb nicht zum Gegenstand der Planung gemacht werden. Es gilt deshalb eine Balance zu finden zwischen einerseits der notwendigen strikten Planung der Planung und der für die Wahrnehmung möglichst aller Planungsgegenstände wünschenswerten Offenheit der Planung.

\section{Kontrollprozess}

Ausgehend von einem Menschenbild, wonach sich der Mensch zugunsten von Zielen, denen er sich verpflichtet fühlt, der Selbstdisziplin und der -kontrolle unterwirft (McGregor, S. 47ff. und 61ff.), sollte Kontrolle möglichst in Form der Selbstkontrolle durch den Verantwortlichen stattfinden. Der Controller unterstützt den für das Erreichen der vereinbarten Ziele Verantwortlichen, indem er diesem die kontrollrelevanten Informationen zur Verfügung stellt. Nur dann, wenn das tatsächliche Ergebnis und/oder das tatsächliche Verhalten der Verantwortlichen erheblich von den gewünschten Größen abweichen und es möglicherweise zu einer Gefährdung des Unternehmens insgesamt kommen könnte, wird die Selbst- durch die Fremdkontrolle ersetzt.

Das Krankenhaus ist in eine überbetriebliche Rahmenordnung integriert. Diese erwartet von dem Krankenhaus bestimmte Handlungen - mit unterschiedlicher Verbindlichkeit - oder verbietet andere. Auch in diesem Zusammenhang gilt das Primat der Selbstkontrolle, deren Glaubwürdigkeit durch ergänzende überbetriebliche Kontrollen gesteigert werden kann. Verschiedene Kontrollinstanzen kommen in Betracht: wie unter anderem der Staat ${ }^{51}$, die Öffentlichkeit, vertreten durch diverse Medien, oder Verbraucherschutzorganisationen.

51 So gibt es zum Beispiel diverse gesetzliche Regelungen, die beim Betrieb von Röntgeneinrichtungen zur Behandlung von Menschen zu beachten sind. Deren Einhaltung wird in Berlin durch das Landesamt für Arbeitsschutz, Gesundheitsschutz und technische Sicherheit (LAGetSi) Berlin kontrolliert. 
Mit der Realisierung eines Führungskonzepts, das auf Selbstkontrolle setzt, unterstreicht die Geschäftsführung die Rolle der Führungskräfte und Mitarbeiter als mündige Stakeholder. Mit der Selbstkontrolle betont die Geschäftsführung ein hohes Maß an Wertschätzung für die Verantwortlichen. Sie schafft die Voraussetzungen dafür, dass im Kontrollprozess die Bedürfnisse der diversen Stakeholder - weil der Kontrollierende nahe am betrieblichen Geschehen verortet ist - angemessen berücksichtigt werden können.

\section{Organisation}

\section{a) Charakterisierung}

Als weitere Managementfunktion, mit deren Hilfe die Voraussetzungen für das Realisieren des Managements der sozialen Verantwortung geschaffen werden können, wurde die Organisation identifiziert, die verschieden definiert werden kann:

1. Zum einen versteht man darunter eine Teilfunktion betrieblicher Leitungstätigkeit. Diese Funktion dient der zielgerichteten Kombination der Produktionsfaktoren und dem Entwerfen der Ordnung, die für das Zusammenwirken der Aufgabenträger in einem multipersonal und arbeitsteilig organisierten Unternehmen notwendig sind.

2. Mit dem Begriff „Organisation“ wird auch das Ergebnis des Organisierens gemäß Ziffer 1. bezeichnet.

Unter Organisation wird hier entsprechend der Definition in Ziffer 2. im Sinne der Mikro-Struktur des Unternehmens die Arbeitsorganisation verstanden (Staehle, S. 675) (Ein Ausschnitt der Unternehmensorganisation auf der Makro-Ebene wird in Kapitel 6.4.4 unter der Überschrift Organisation der Leitungstätigkeit behandelt).

Bei der schon behandelten Managementfunktion Controlling und der im Kapitel 6.4.3, Personalarbeit, noch zu untersuchenden Teilfunktion Personalarbeit wurde bzw. wird danach gefragt werden, wie die Managementfunktion gestaltet werden muss, um der Ceschäftsführung, den Führungskräften und den Mitarbeitern des Krankenhauses ein sozial verantwortetes Handeln dauerhaft zu ermöglichen. Im Zusammenhang mit der Managementfunktion Organisation wird dagegen eine Antwort auf die Frage gesucht, wie die Organisation des Unternehmens als Ergebnis der Teilfunktion betrieblicher Leitungstätigkeit beschaf- fen sein muss, damit diese der geeignete Rahmen für die Realisierung des Managements der sozialen Verantwortung ist.

Von der Festlegung organisatorischer Regelungen sind aber nicht nur die Geschäftsführung sowie die Mitarbeiter und deren Führungskräfte, sondern auch viele andere Stakeholder des Krankenhauses betroffen. Auch deren Entscheidungsspielräume, deren Spielraum für die Realisierung ihrer eigenen Ziele wird möglicherweise nicht unwesentlich beeinflusst (s. Beispiele 1 und 2).

\section{Beispiel 1}

Ein Krankenhausträger, der in einem Ballungsgebiet mehrere Krankenhäuser betreibt, beschließt, vergleichbare medizinische Leistungen, die sich durch hohe Anforderungen an die Qualifikation der dafür zuständigen Ärzte auszeichnen und die bisher in nahezu allen der von ihm betriebenen Krankenhäusern angeboten werden, in jeweils einem Behandlungszentrum zusammenzufassen. Für chronisch kranke Patienten, die auf diese Leistungen angewiesen sind, bedeutet dieses, dass sie künftig entweder ein anderes Krankenhaus in ihrer Nachbarschaft mit einem entsprechenden Leistungsangebot aufsuchen oder dass sie, um den ihnen vertrauten Arzt weiterhin konsultieren zu können, einen - in zeitlicher und in finanzieller Hinsicht sehr viel aufwändigeren Weg zu dem neuen Behandlungszentrum in Kauf nehmen.

\section{Beispiel 2}

Das Krankenhaus beschließt, bestimmte Materialien künftig nicht mehr zu bevorraten, sondern sich im Bedarfsfall gewissermaßen „just-in-time“ beliefern zu lassen. Für die davon betroffenen Lieferanten bedeutet dieses, sich organisatorisch auf diese Form der Belieferung einzustellen oder - wenn dieses nicht erfolgen soll - möglicherweise einen Kunden zu verlieren.

Um die Gestaltungs-Felder der Organisation identifizieren zu können, bedarf es eines Blickes auf den Prozess des Entstehens der Organisation. Die Schritte dieses Prozesses sind (Kosiol, S. Goff.)

1. die Zerlegung der Gesamtaufgabe des Krankenhauses in Teilaufgaben und

2. die Zusammenfassung gleichartiger Teilaufgaben zu der Aufgabe einer Stelle und in weiterer Folge zu den Aufgaben einer größeren Organisationseinheit - wie z.B. einer klinischen Abteilung -, deren Wahrnehmung einem Mitglied der Geschäftsführung, einer 
Führungskraft oder einem Mitarbeiter übertragen wird.

Gleichartig in diesem Sinne sind die Aufgaben hinsichtlich bestimmter Merkmale, und diese sind

- die zwecks Erledigung einer Aufgabe auszuführende Verrichtung,

- das Objekt, an dem die Verrichtungen vollzogen wird,

- der Rang der Teilaufgabe als Entscheidungsoder als ausführende Aufgabe sowie

- die Zeit, der Ort und die Hilfsmittel für die Erledigung der Aufgabe.

Bei der Zusammenfassung gleichartiger Aufgaben zu Stellenaufgaben kommen grundsätzlich vier Gestaltungsprinzipien und Arten von Spezialisierungen in Betracht:

1. Zusammenfassung von Teilaufgaben mit gleichartigen Verrichtungen, die an allen Arten von Objekten ausgeführt werden (s. Beispiel 3).

\section{Beispiel 3}

Jede Pflegekraft übernimmt aus dem Katalog der Pflegearbeiten jeweils eine Funktion - wie zum Beispiel das Messen des Blutdrucks - und führt diese bei allen Patienten einer klinischen Abteilung aus. Die auf diese Weise organisierte Pflege wird als Funktionspflege bezeichnet.

2. Zusammenfassung von Teilaufgaben mit unterschiedlichen Verrichtungen, die für gleichartige Objekte wahrgenommen werden (s. Beispiel 4).

\section{Beispiel 4}

Einer Pflegekraft wird eine kleine Gruppe von Patienten mit gleichartigen Erkrankungen zugeordnet. Bezogen auf diese Patienten führt die Pflegekraft alle notwendigen Pflegemaßnahmen durch. In der Praxis wird das Ergebnis dieses Gestaltungsprinzips - unter dem Namen Gruppenpflege im Regelfall nicht auf eine einzelne Pflegekraft, sondern auf eine Gruppe von Pflegekräften als Teil der Pflegenden einer klinischen Abteilung angewendet.

3. Zusammenfassung von Entscheidungsaufgaben einschließlich der Befugnis, das Ergebnis der Entscheidung anderen zur Ausführung anzuweisen, bei einer Stelle und Zusammenfassung der Ausführungsaufgaben bei anderen Stellen.
4. Eine spezielle Form der Arbeitsteilung gibt es zwischen den Organisationsbereichen Linie und Stab. Die Spezialisten des Stabes bereiten eine Entscheidung vor, indem sie die für die Lösung eines Problems infrage kommenden Alternativen generieren und bewerten. Der Linienverantwortliche wählt die Alternative aus, die umgesetzt werden soll; er trägt damit die Verantwortung für das Ergebnis des Entscheidungsprozesses und dessen Umsetzungs-Folgen.

Die Weiter-Entwicklung der Strukturen eines Krankenhauses ist vor allem durch den Fortschritt der Medizin und die der Medizin-Technik getrieben. Der Grad der Spezialisierung der Führungskräfte und der Mitarbeiter und in deren Folge der Grad der horizontalen Arbeitsteilung - im Sinne sowohl der verrichtungs- als auch der objektorientierten Spezialisierung - und der vertikalen Arbeitsteilung nehmen zu. Die Folgen davon sind unter anderem:

1. Patienten werden immer häufiger von einer größeren Zahl von Spezialisten behandelt, deren Entscheidungsspielräume und Verantwortungsbereiche - bezogen auf die gesamte Behandlung des Patienten - immer mehr eingeschränkt werden. Der Arzt, der für die Behandlung des Patienten gesamtheitlich verantwortlich ist und dem der Patient vertrauen will und kann, gibt es in einer solchen Struktur möglicherweise nicht mehr. Nicht selten kommt es vor, dass der Patient während der Dauer seines Aufenthaltes im Krankenhaus bei der täglichen Visite von jeweils verschiedenen Ärzten besucht wird.

2. Auf der anderen Seite kann damit gerechnet werden, dass in Folge dieser Spezialisierung die Qualität der Behandlung und die Behandlungssicherheit, aber auch die Arbeitsproduktivität und damit die Effizienz der Behandlung zunehmen.

3. Die Arbeit des Arztes wird weniger abwechslungsreich, wenn er zum Beispiel in einer kardiologischen Klinik - als Ergebnis einer sowohl verrichtungs- als auch objektorientierten Spezialisierung - nur für die Durchführung der Ultraschalluntersuchungen zuständig ist.

Die verrichtungsorientierte Spezialisierung im Pflegedienst - dort als Funktionspflege bekannt - führt zur Monotonie der Arbeit der Pflegekräfte und dazu, dass die Patienten nicht mehr nur einen Ansprechpartner und damit 
die Vertrauensperson im Pflegedienst haben, sondern im Tagesablauf von mehreren Mitarbeitern des Pflegedienstes versorgt werden.

4. Weil der einzelne Mitarbeiter nur noch für einen Ausschnitt der Behandlung eines Patienten verantwortlich ist, nimmt die Gefahr $\mathrm{zu}$, dass diese Teil-Verantwortung nicht mehr konsequent genug wahrgenommen wird der nächste Mitarbeiter in der Behandlungskette wird schon aufpassen.

5. Die Gesundung des Patienten kann nicht mehr einem einzelnen Mitarbeiter als besonderer Behandlungserfolg zugeschrieben werden.

6. Die Spezialisten nehmen nur noch einen kleinen Ausschnitt ihrer Umwelt wahr und damit auch nicht die Folgen ihres Handelns für die Betroffenen.

7. Bei Fehlentwicklungen versuchen sowohl die Führungskraft, die eine Entscheidung fällt, als auch deren Mitarbeiter, der die Entscheidung vorbereitet, sich mit dem Hinweis zu exkulpieren, sie hätten sich auf die sachkundige Vorbereitung der Entscheidung durch den Mitarbeiter verlassen (können) (die Führungskraft) bzw. er hätte nur beraten und nicht entschieden (der Mitarbeiter).

Es ist im Sinne des Managements der sozialen Verantwortung, wenn den Patienten die Errungenschaften der Medizin und die der Medizin-Technik ohne Einschränkung zugänglich gemacht werden. Dabei muss allerdings bedacht werden, dass organisatorische Gestaltungsoptionen gefunden werden, die der Realisierung der Grundidee des Managements der sozialen Verantwortung nicht im Wege stehen. Es gilt, eine Balance zu finden zwischen den ökonomischen Vorteilen der Arbeitsteilung und dem Befriedigen diverser Stakeholder-Bedürfnisse. Es gilt Barrieren abzubauen, die sozial verantwortliches Handeln erschweren (Göbel 2006, S. 234).

Empirische Untersuchungen haben gezeigt (Waters, S. 283ff.), dass es insgesamt sieben ,organizational blocks" gibt, die sozial verantwortliches Handeln behindern können. Drei von diesen 1. division of work,

2. strict line of command und

3. separation of decision

sind auf die bisher dargestellten Formen der Arbeitsteilung und deren Folgen zurückzuführen. Sie sollen hier - auch auf die Gefahr hin, einiges zu wiederholen - kurz beschrieben werden (Oppenrieder, S. 25ff. und die dort genannte Lite- ratur), um anschließend - indem Anforderungen an die Organisation des Krankenhauses formuliert werden - Wege zu deren Überwindung aufzuzeigen.

\section{Zu 1. division of work}

Das Ergebnis der horizontalen Arbeitsteilung ist die mehr oder weniger starke Zergliederung des Krankenhauses in kleinere und größere Organisationsbereiche, die an der Behandlung eines $\mathrm{Pa}$ tienten entweder unmittelbar beteiligt sind oder als medizinische oder nicht-medizinische Supportbereiche Leistungen bereitstellen, die für die erfolgreiche Behandlung des Patienten erforderlich sind. Die für die genannten Organisationsbereiche verantwortlichen können die Gesamtfolgen ihres Handelns für den Adressaten der Verantwortung häufig nicht mehr übersehen; Ressortdenken führt dazu, dass unsoziale Praktiken nicht wahrgenommen und deshalb auch nicht geändert werden, weil sie nicht in den eigenen Verantwortungsbereich fallen.

\section{Zu 2. strict line of command}

Es gibt eine eindeutige Einlinienorganisation mit widerspruchsfreien Anweisungs- und Informationswegen. Jeder Mitarbeiter hat nur einen Vorgesetzten. Es besteht die Gefahr des Abschiebens von Verantwortung und des Entlastens von der Verantwortung für die Folgen ihres Handelns. Die Mitarbeiter können sich auf den „Befehlsnotstand“ berufen; sie erledigen nur das, was ihnen von der Führungskraft aufgetragen worden ist. Die Weisungen der Führungskraft werden nicht hinterfragt. Missliebige Informationen werden nicht weitergeleitet.

Die Führungskraft kann sich der Verantwortung mit der Begründung entziehen, dass sie einerseits auf Anweisungen ihrer vorgesetzten Instanzen tätig wird und dass sie andererseits mit ihren Mitarbeitern Zielvereinbarungen abgeschlossen hat, die für das Erreichen der Ziele zu fällenden Mittel-Entscheidungen aber in der Verantwortung der Mitarbeiter liege.

Es gibt eine Tendenz zu einer großen Zahl von Leitungsebenen mit einer zunehmenden Zahl von Störfaktoren für die Kommunikation. Das Maß an Verantwortung, das in den einzelnen Leitungsebenen von den Führungskräften und den Mitarbeitern wahrzunehmen ist, erscheint immer geringer. Die Bereitschaft, sich von der Übernahme der Verantwortung „zu drücken“, nimmt zu. 


\section{Zu 3. separation of decision}

Eine spezielle Form horizontaler Arbeitsteilung ist die separation of decision: die Trennung des Entscheidungsprozesses in

a) die Entscheidungsvorbereitung und

b) die Auswahl der zu realisierenden Alternative.

Die Zuweisung dieser beiden Aufgaben zu einem Spezialisten, der dem Verantwortungsträger zuarbeitet (Aufgabe a), und zu dem Verantwortungsträger (Aufgabe b) führt zur Delegation von Verantwortung von dem einen zu dem anderen Aufgabenträger und umgekehrt und dazu, dass Verantwortung nicht mehr wahrgenommen wird. Die im formalen Sinne kompetenzlosen Experten sind sich ihrer Verantwortung nicht bewusst; den formal zuständigen Verantwortungsträgern fehlt das Fachwissen, um ein kritisches Verantwortungsbewusstsein entwickeln und Fehlentwicklungen erkennen zu können.

\section{b) Anforderungen an die Organisation}

Aus den bisherigen Ausführungen lassen sich drei Gestaltungs-Felder identifizieren, bei denen die Chance besteht, dass durch die Art der Organisation die Wahrnehmung von Verantwortung unterstützt werden kann. Es sollte damit die Möglichkeit gegeben sein, einen Teil der erwähnten Nachteile, die sich aus den zu erwartenden strukturellen Veränderungen ergeben, zu beseitigen.

\section{Die drei Gestaltungs-Felder sind}

1. der Entscheidungsspielraum, der den Führungskräften und den Mitarbeitern jeweils zugebilligt wird,

2. die horizontale Arbeitsteilung (bisher als verrichtungs- und als objektorientierte Spezialisierung bezeichnet) und

3. die vertikale Arbeitsteilung (als Ergebnis der Unterscheidung der Aufgaben nach ihrem Rang in Entscheidungsaufgaben mit Weisungsbefugnis und in ausführende Aufgaben und der Verteilung der Phasen des Entscheidungsprozesses auf verschiedene Personen).

Aus der Crundidee des Managements der sozialen Verantwortung und unter Berücksichtigung der Grundpostulate lassen sich, differenziert für die genannten Gestaltungs-Felder, die nachfolgend beschriebenen Anforderungen an die Gestaltung der Organisation entwickeln.

\section{Anforderungen an die Organisation}

\section{Gestaltungs-Feld, Anforderungen an die Gestaltung}

\section{Entscheidungsspielraum der Führungskräfte und der Mitarbeiter}

Im Sinne der Grundidee des Managements der sozialen Verantwortung werden Entscheidungs- und Tätigkeitsspielräume geschaffen, die dem Akteur die Möglichkeit geben, im Rahmen der Erfüllung der ihm übertragenen Aufgabe für die Lösung eines Problems Alternativen zu entwickeln, diese unter Berücksichtigung der Interessen der von seiner Entscheidung betroffenen Stakeholder zu bewerten und eine der Alternativen für die Umsetzung auszuwählen, also sozial verantwortlich zu handeln.

Wenn die Entscheidungsspielräume erweitert werden müssen, weil sie sonst für die Wahrnehmung von Verantwortung nur bedingt geeignet sind, stehen grundsätzlich drei Alternativen zur Verfügung (Scholz, S. 515 und $616 \mathrm{ff}$.):

- job rotation,

- job enlargement und

- job enrichment.

Aus der Sicht des Managements der sozialen Verantwortung sind die genannten Instrumente unterschiedlich zu beurteilen (Wittmann, S. 148f.). Job rotation und job enlargement dienen durch den Wechsel auf einen anderen Arbeitsplatz bzw. durch die Hinzunahme weiterer, qualitativ gleichwertiger zu den schon wahrgenommenen Aufgaben vielfach in erster Linie dazu, Monotonie und einseitige Arbeitsbelastungen zu reduzieren - auch das wäre im Sinne der Führungskräfte und Mitarbeiter. Eine Erweiterung des Entscheidungsspielraumes findet in der Regel aber nicht wirklich statt.

Eine Bereicherung der Aufgabeninhalte durch das Hinzufügen von Planungs- und Kontrollaufgaben erfolgt dagegen bei job enrichment. Insofern kann auch nur mittels des job enrichment ein wirklicher organisatorischer Durchbruch, also eine Erweiterung des Entscheidungsspielraums, erreicht werden. Der Erfolgsorientierung des Managements der sozialen Verantwortung wird entsprochen. Das Bedürfnis nach Entfaltung der eigenen Persönlichkeit der von den organisatorischen Veränderungen betroffenen Verantwortungsträger wird berücksichtigt. Deren Mündigkeit wird gefördert.

\section{Horizontale Arbeitsteilung}

Die erwähnten Folgen der division of work können - dann aber möglicherweise zu Lasten der Effizienz - überwunden werden, wenn einzelne Funktionen zu ganzheitlichen Arbeitsprozessen zusammengeführt werden. Einem Team von mehreren Personen - eventuell mit unterschiedlichen Fähigkeitsprofilen - wird die Wahrnehmung eines 
vollständigen Aufgabenkomplexes einschließlich der dafür notwendigen Planungs- und Kontrollkompetenzen übertragen; es entsteht eine teilautonome Arbeitsgruppe ${ }^{52}$. In der Praxis der stationären Pflege wird das Ergebnis dieser Art von horizontaler Arbeitsteilung als Gruppenpflege bezeichnet.

Eine Alternative ist eine mehr am Behandlungsprozess orientierte Organisation. Die horizontale Arbeitsteilung wird als objektorientierte Spezialisierung vom Endergebnis des Behandlungsprozesses ausgehend gedacht und organisiert. Damit kann ein Verantwortlicher für das Endergebnis der Patientenbehandlung benannt werden. Die Zahl der Schnittstellen und das Fehlerrisiko können reduziert werden.

Mittels beider Organisationsformen wird erreicht, dass Führungskräfte und Mitarbeiter ein höheres Maß an Autonomie erhalten; es wird ihnen Vertrauen in ihre Fähigkeit, Verantwortung zu übernehmen, signalisiert. Dem Eigenwert und der Subjektgeltung der Führungskräfte und der Mitarbeiter wird Rechnung getragen (Göbel 2006, S. 236f.). Die Mündigkeit der Verantwortungsträger wird gefördert. Dem Bedürfnis der Patienten nach weiterer Steigerung der Behandlungsqualität und -sicherheit wird entsprochen.

\section{Vertikale Arbeitsteilung (= Hierarchisierung)}

Die organisationale Barriere „strict line of command“ kann überwunden werden durch den Abbau überflüssiger hierarchischer Ebenen und durch die Zusammenführung von Planungs- und Handlungsautorität sowie von Verantwortung bei jeweils einem Entscheidungsträger. Mit der Dezentralisierung der Leitungsarbeit wird den Führungskräften und ihren Mitarbeitern die Verfügungsgewalt über Ziele und Mittel übertragen. Der Entscheidungsspielraum und damit die Möglichkeit, Verantwortung zu übernehmen, werden erweitert.

Im Übrigen wird das Bedürfnis der Führungskräfte an der Basis des Krankenhauses und deren Mitarbeiter nach Wertschätzung und Selbstentfaltung befriedigt. Sie sind nicht mehr nur Ausführungsorgan anderswo beschlossener Maßnahmen (Wittmann, S. 160).

Um ein Abschieben von Verantwortung von der Führungskraft auf die Experten oder umgekehrt verhindern zu können - siehe „separation of decision“ -, sollte diese Form von Arbeitsteilung möglichst auf ein Mindestmaß

52 Diverse Automobilhersteller haben Anfang der siebziger Jahre diese Form der Arbeits-Organisation unter der Bezeichnung „Werkstattfertigung“ eingeführt. Der Nachteil ist eine - im Vergleich zur Fließbandfertigung - geringere Arbeitsproduktivität. In der Zwischenzeit wurde diese Form der Arbeitsorganisation mit dem Ziel der Effizienzsteigerung und unter Hintanstellung der Vorteile für die beteiligten Mitarbeiter wieder aufgegeben. reduziert werden. Besser ist, die Entscheidungsbefugnis möglichst weit an die Basis zu verlagern, nämlich dorthin, wo das Expertenwissen verortet ist.

\section{Personalarbeit}

\section{a) Charakterisierung, Ziele und Felder des Personalmanagements}

Im Kapitel 6.4.3 wird zunächst danach gefragt, wie die Teilfunktion betrieblicher Leitungstätigkeit „Controlling“ und das Ergebnis der Teilfunktion betrieblicher Leitungstätigkeit „Organisation“ beschaffen sein müssen, damit Verantwortung im Sinne des Managements sozialer Verantwortung von einem Verantwortungsträger erfolgreich wahrgenommen werden kann. Das Wahrnehmen von Verantwortung bedingt allerdings nicht nur die schon genannten institutionellen Voraussetzungen, sondern auch der Unterstützung durch eine spezifische Art von Personalarbeit. Letztere wird nunmehr zum Thema gemacht.

Die Ziele des Personalmanagement werden aus den Zielen des Unternehmens abgeleitet und leisten, wenn sie erreicht werden, einen Beitrag zur Realisierung der Unternehmensziele, nämlich für die langfristige finanzielle Sicherung der Krankenhaus-Existenz sowie für den Erhalt der in ihm vorgehaltenen Arbeitsplätze. Diese Ziele sind dann realisierbar, wenn unter anderem gewährleistet ist, dass die Kunden des Krankenhauses - die Patienten und die einweisenden Ärzte mit den Leistungen des Krankenhauses zufrieden sind, und wenn auf dieser Crundlage mit einer dauerhaft ausreichenden Inanspruchnahme mit ausreichend hohen Erlösen gerechnet werden kann. Zufriedenheit der Kunden setzt eine auf eine entsprechende Struktur- und Prozessqualität gestützte hohe Behandlungs- und Servicequalität sowie ein gutes Preis-Leistungs-Verhältnis voraus.

Die zuletzt genannten Ziele lassen sich verwirklichen, wenn das Personalmanagement

- bedarfsgerecht qualifizierte und motivierte Mitarbeiter gewinnen, an das Krankenhaus binden und wirtschaftlich einsetzen kann und

- wenn es als Voraussetzung dafür Arbeitsbedingungen schafft, die als Anreize für die Führungskräfte und Mitarbeiter dafür sorgen, dass diese ihre Fähigkeiten effektiv und effizient im Sinne der genannten Ziele mobilisieren und umsetzen. 
Die Realisierung der erwähnten Ziele wird jedoch erschwert, solange die nachstehend genannten strukturellen Konflikte, durch die die Personalarbeit in einem Krankenhaus gekennzeichnet ist, nicht überwunden werden können.

- Krankenhäuser setzen Mitarbeiter ein, um die Existenz des Krankenhauses sichern und damit die Voraussetzung für die dauerhafte Befriedigung des Bedarfs an medizinischen Leistungen schaffen zu können. Die Mitarbeiter dagegen stellen ihre Arbeitskraft dem Krankenhaus zur Verfügung, um erstens ihre persönlichen Bedürfnisse (unter anderem die Sicherung ihrer Existenz und die eines ausreichend hohen Einkommens) und zweitens ihre Rolle als Agent der Patienten erfüllen zu können.

- Die Personalarbeit ist darüber hinaus geprägt durch die Sonderstellung der Leitenden Ärzte, die im Rahmen ihrer privaten Tätigkeit im Krankenhaus eigene Ziele verfolgen.

- Personalführung im Krankenhaus ist im Regelfall Gruppenführung. Deren spezifisches Ziel ist die Steigerung der Teamleistung durch das Nutzen von Synergien innerhalb der Cruppe (Scholz, S. 849). Der Erfolg der Gruppe wird getragen durch die gegenseitigen Sympathien der Cruppenmitglieder und durch deren Motivation, in der Gruppe bleiben zu wollen. Die Cruppe schützt die Gruppenmitglieder gegen unerwünschte Einflüsse von außen. Der Druck innerhalb der Gruppe auf jedes ihrer Mitglieder kann aber beträchtlich sein und damit die Leistung des Teams empfindlich schmälern.

Die Überwindung dieser Konflikte durch das Personalmanagement setzt voraus, dass die verschiedenen Funktionen des Personalmanagements so gestaltet werden, dass die Verantwortungsträger bereit und imstande sind, Verantwortung im Sinne der Goldenen Regel wahrzunehmen. Verantwortungsträger im Sinne des Personalmanagements sind vor allem die Führungskräfte; die Personalabteilung stellt die Personalmanagement-Instrumente zur Verfügung und unterstützt die zuerst genannten Verantwortungsträger bei deren Anwendung.

Als Crundlage für die Beschreibung der Aufgaben der Personalarbeit und für die Identifizierung der Anforderungen an diese (s. folgenden Textabschnitt) soll eine Gliederung der Aufgaben verwendet werden, die von dem inneren Zusam-
Tab. 15 Felder des Personalmanagements

\begin{tabular}{|c|c|}
\hline Personalbedarfs- & Personaleinsatz \\
\hline bestimmung & Personalkostenmanagement \\
\hline Personalbestandsanalyse & Personalcontrolling \\
\hline $\begin{array}{l}\text { Personalveränderung } \\
\text { Personalbeschaffung } \\
\text { Personalentwicklung } \\
\text { Personalfreisetzung }\end{array}$ & Personalführung \\
\hline
\end{tabular}

menhang dieser Aufgaben ausgeht. Personalmanagement wird als ein Prozess aufgefasst (Scholz, S. 83ff.), dessen Elemente miteinander integriert sind. Cleichartige Aufgaben werden Feldern des Personalmanagements zugeordnet (s. Tab. 15).

\section{b) Anforderungen an ein Personalmanagement der sozialen Verantwortung}

Anforderungen an ein Personalmanagement der sozialen Verantwortung stellen sich in allen Feldern des Personalmanagement, und zwar wie folgt.

\section{Anforderungen an das Personalmanagement}

\section{Gestaltungs-Feld, Anforderungen an die Gestaltung}

\section{Personalbedarfsbestimmung}

Das Ergebnis der Personalbedarfsbestimmung ist einerseits die Zahl der Mitarbeiter, die für die Realisierung eines konkreten Leistungsprogramms benötigt werden. Zum anderen fragt sie danach, welchen Anforderungen diese Mitarbeiter genügen müssen.

Der Bedarf an Mitarbeitern hängt - in quantitativer Hinsicht - entscheidend davon ab, wie die Interessen der von dem Ergebnis der Personalbedarfsbestimmung betroffenen gewichtet werden. Er wird größer sein, wenn die Anliegen der Patienten und die der Mitarbeiter bei der einschlägigen Entscheidung mit hohem Gewicht berücksichtigt werden; er wird dagegen geringer dimensioniert sein, wenn die Interessen des Eigentümers und der Krankenkassen eine gewichtigere Rolle spielen. Die Verantwortung darüber, mit welchem Gewicht die Stakeholder-Anliegen bei den einschlägigen Entscheidungen berücksichtigt werden sollen, ist im Regelfall der Geschäftsführung zugewiesen. Diese legt als Ergebnis einer dialogischen Verständigung mit den Betroffenen und als eine Art Vor-Entscheidung die Stakeholder-Anliegen-Gewichtung fest (Näheres zu der Gewichtung von Stakeholder-Anliegen s. Kap. 8.3), bevor der Prozess der Personalbedarfsbestimmung gestartet werden 
kann. Das Ergebnis dieser Vor-Entscheidung ist für alle Verantwortungsträger verbindlich.

Die notwendige Qualifikation der Mitarbeiter ist einerseits den Aufgaben geschuldet, die die Mitarbeiter wahrnehmen sollen; sie ist Gegenstand des Anforderungsprofils. Um dem Management der sozialen Verantwortung dauerhaft zur Wirksamkeit verhelfen zu können, werden in das Anforderungsprofil zusätzlich zu den üblichen Anforderungen fachlicher, methodischer und sozialer Art auch die Fähigkeit und die Bereitschaft, sozial verantwortlich zu handeln, aufgenommen; dazu gehören zum Bejspiel Dialogfähigkeit, Toleranz und die Fähigkeit zu einem Verhalten, das durch Achtung gegenüber den Bedürfnissen anderer gekennzeichnet ist, sowie zur argumentativen, dialogorientierten Auseinandersetzung (Wittmann, S. 181).

Wird das Personalmanagement in den Dienst des Managements der sozialen Verantwortung gestellt, dann hat dieses auch Auswirkungen auf die Gestaltung des Prozesses der Personalbedarfsbestimmung, und zwar in zweierlei Hinsicht:

- Der Prozess sollte als Gegenstromverfahren organisiert werden (Naegler et al., S. 113ff.). Damit wird gewährleistet, dass das Ergebnis der Personalbedarfsbestimmung das Ergebnis einer argumentativen, dialogischen Verständigung unter allen von den Folgen der Personalbedarfsbestimmung betroffenen ist und dass die Bedürfnisse der betroffenen Verantwortungsträger nach einer angemessenen Personalausstattung berücksichtigt werden, und zwar sowohl in Bezug auf die Zahl der Mitarbeiter als auch auf deren Qualifikation.

- Die Anforderungen an den Mitarbeiter, dem eine Aufgabe zur Wahrnehmung übertragen wird, müssen von der für diesen Mitarbeiter verantwortlichen Führungskraft so rechtzeitig festgelegt werden, damit sie bei dem Prozess der Personalbedarfsbestimmung berücksichtigt werden können.

Durch das Einbeziehen der Mitarbeiter, die das Ergebnis der Planung umsetzen werden, in den Planungsprozess ist gewährleistet, dass die Bedürfnisse der Mitarbeiter und der Patienten berücksichtigt werden können. Die Mündigkeit der an dem Prozess beteiligten Führungskräfte und Mitarbeiter wird gefördert. Die Geschäftsführung bringt den Führungskräften und den Mitarbeitern ein hohes Maß an Wertschätzung zum Ausdruck. Sie kommt ihrer Begründungspflicht nach.

\section{Personalbestandsanalyse und -veränderung}

Der Vergleich des aktuellen Personalbestands mit dem Ergebnis der Personalbedarfsbestimmung wird durchgeführt, um sicherstellen zu können, dass

- die für die Realisierung des vereinbarten Leistungsprogramms ausreichende und mit den Bedürfnissen der Stakeholder abgestimmte Zahl von Mitarbeitern zur Verfügung steht,

- die für die Wahrnehmung der übertragenen Aufgabe und für sozial verantwortliches Handeln erforderliche Qualifikation der Mitarbeiter gegeben ist bzw. um - eventuell bestehende Defizite beseitigen zu können. Die Personalbestandsanalyse kann sich auf das Krankenhaus insgesamt und/oder einzelne seiner Organisationseinheiten beziehen. Zum anderen werden einzelne Mitarbeiter aus unterschiedlichen Gründen daraufhin beurteilt, ob deren Verhalten und Leistungen den in sie gesetzten Erwartungen entsprechen.

In Abhängigkeit von der Art der Differenz - und das gilt unabhängig von der Art der Analyse-Anlässe - werden entweder neue Mitarbeiter rekrutiert und/oder vorhandene weiterentwickelt und/oder Mitarbeiter freigesetzt (Naegler et al., S. 134ff.):

- Wenn neue Führungskräfte und/oder Mitarbeiter rekrutiert werden müssen, weil es einen Personal-Fehlbestand in quantitativer Hinsicht gibt, stellen sich aus der Sicht des Managements der sozialen Verantwortung spezifische Anforderungen an das Auswahlverfahren. Dieses beginnt mit der möglichst präzisen Formulierung des Anforderungsprofils (zu den Inhalten siehe Feld Personalbedarfsbestimmung) in der Stellenausschreibung. Das Anforderungsprofil dient im Bewerbergespräch als Grundlage für eine argumentative, dialogische Verständigung zwischen dem Bewerber und seinem künftigen Vorgesetzten über die Art der ausgeschriebenen Aufgabe und die der Arbeitsbedingungen. Es wird außerdem als Grundlage für eine fundierte Begründung der Personalauswahl-Entscheidung benötigt. Schließlich wird es gebraucht, um den nicht zum Zuge gekommenen Bewerbern - wenn diese dieses möchten - die Gründe für ihre Ablehnung mitteilen zu können.

- Wenn das erwähnte Defizit qualitativer Art ist, steht das Personalmanagement vor folgender Konfliktsituation:

- Das Defizit kann beseitigt werden, indem die nicht ausreichend qualifizierten Mitarbeiter freigesetzt und neue Mitarbeiter mit den richtigen Fähigkeiten eingestellt werden.

- Das Defizit kann aber auch dadurch behoben werden, indem die nicht ausreichend qualifizierten Mitarbeiter - sofern diese Mitarbeiter über die notwendigen Entwicklungspotenziale verfügen entsprechend den neuen Anforderungen weiterentwickelt werden. Dabei ist Personalentwicklung häufig nicht nur eine fachliche Weiterentwicklung (einschließlich der Entwicklung der dazugehörigen methodischen und sozialen Kompetenzen), sondern auch eine Einstellungs-Entwicklung (Näheres zu der Personalentwicklung s. Kap. 7.3.3 und 7.4.1). 
Bei der Entscheidung, welchem dieser Lösungsansätze der Vorrang eingeräumt werden soll, spielen - weil die verschiedenen Vorgehensweisen unterschiedlich hohe Kosten verursachen - die Bedürfnisse des Eigentümers und der Krankenkassen nach Generierung eines möglichst hohen Gewinns und Minimierung der Kosten, aber auch die Bedürfnisse der Mitarbeiter nach Sicherheit ihres Arbeitsplatzes und einer angemessenen Vergütung sowie nach Wertschätzung eine Rolle und werden im Sinne sozial verantwortlichen Handelns explizit und begründbar berücksichtigt.

\section{Anmerkung:}

Im Vergleich zu der Personalbedarfsbestimmung sind die Gewichte der Anliegen der erwähnten Stakeholder neu zu bestimmen und auszubalancieren. Schließlich geht es den Mitarbeitern nicht mehr nur um eine möglichst auskömmliche Personalausstattung - wie bei der Personalbedarfsbestimmung. Ihr Anliegen ist jetzt existenzieller Art und bedarf deshalb einer deutlich höheren Gewichtung gegenüber den Anliegen der Eigentümer und Krankenkassen.

- Wenn ein Zuviel an Personal registriert wird und das Krankenhaus sich von Mitarbeitern trennen muss, stellen sich - ausgehend von den Bedürfnissen der Mitarbeiter und unter Berücksichtigung der Beschränkung des Gewinnprinzips - Anforderungen an das Personalmanagement der sozialen Verantwortung in drei Dimensionen:

- Einerseits stellt sich die Frage, ob durch eine andere Gewichtung der den Personalbedarf beeinflussenden Stakeholder-Interessen - die Gewinnerzielungsabsicht wird weniger hoch gewichtet - oder durch die Veränderung anderer den Personalbedarf bestimmender Größen eine Veränderung des Personalbedarfs und damit die Beseitigung der zuvor festgestellten Differenz erreicht werden kann. In dieser Dimension stellt sich an das Personalmanagement - ausgehend von dem Grundpostulat "Berücksichtigung der Interessen Betroffener" und hier vor allem von dem Bedürfnis nach Arbeitsplatzsicherheit - auch die Forderung nach der Realisierung aller personellen Maßnahmen, die geeignet sind, die Personalfreisetzug i.e.S. vermeiden zu können - dazu zählen unter anderem Arbeitszeitverkürzung, Kurzarbeit oder Einstellungsstopp (Naegler et al., S. 180).

- Die zweite Dimension umfasst - wenn es nach der Nutzung aller anderen personellen Maßnahmen bei einem Zuviel an Personal bleibt und betriebsbedingte Kündigungen notwendig werden - die Forderung nach der sachgerechten Durchführung der Sozialauswahl (Naegler et al., S. 180f.). Die für die Sozialauswahl geltenden Regeln räumen dem
Arbeitgeber einen gewissen Gestaltungsspielraum ein, den dieser auch für die Berücksichtigung der Interessen der von der betriebsbedingten Kündigung betroffenen Mitarbeiter nutzen kann; dabei spielen moralische Prinzipien eine Rolle.

Wie der erwähnte Gestaltungsspielraum genutzt wird, ist das Ergebnis einer argumentativen, dialogischen Verständigung zwischen der Geschäftsführung auf der einen Seite und den Führungskräften und Mitarbeitern auf der anderen Seite. Letztere lassen sich gegebenenfalls durch die Personalvertretung unterstützen und/oder vertreten. Die Personalvertretung muss dem Ergebnis der Sozialauswahl zustimmen.

- Das Personalmanagement ist schließlich gefordert, ausscheidende Mitarbeiter aktiv zu unterstützen, sie zu beraten, gegebenenfalls weiterzubilden und innen bei der Beschaffung eines neuen Arbeitsplatzes möglichst zu helfen. Wenn die Personalfreisetzung durch das Instrument Outplacement unterstützt wird, ist damit am ehesten gewährleistet, dass sowohl die Interessen der ausscheidenden Mitarbeiter als auch die des Unternehmens angemessen berücksichtigt werden.

Wie auch immer sich die Geschäftsführung anlässlich der verschiedenen Entscheidungssituationen positioniert, wichtig ist, dass die Entscheidung auf der Grundlage eines Dialogs mit den Betroffenen herbeigeführt wird und dass das Ergebnis der Entscheidung gegenüber den unmittelbar Betroffenen, aber auch gegenüber allen anderen Mitarbeitern, die sich mit den unmittelbar Betroffenen möglicherweise solidarisieren werden, mit guten Argumenten vertreten werden kann.

\section{Personaleinsatz}

Das Personaleinsatzmanagement führt Mitarbeiter und durchzuführende Aufgaben und die damit verbundene Verantwortung zusammen. Es stellt sicher, dass die Fähigkeiten des einzusetzenden Mitarbeiters mit den Anforderungen der Stelle übereinstimmen, dass die Verantwortung, die einer Stelle zugewiesen worden ist, tatsächlich auch wahrgenommen werden kann.

Eine Besonderheit des Personaleinsatzmanagements sind die drei Arten von Verantwortung, die in diesem Zusammenhang eine Rolle spielen: die Anordnungsverantwortung, die Übernahmeverantwortung und die Durchführungsverantwortung. Die für den Einsatz von Mitarbeitern verantwortliche Führungskraft trägt die Verantwortung dafür, dass der für die Durchführung einer Aufgabe bestimmte Mitarbeiter zu deren Wahrnehmung befähigt ist (= Anordnungsverantwortung), während der beauftragte Mitarbeiter für die sach- und zeitgerechte Durchführung 
der Aufgabe verantwortlich ist (= Übernahme- und Durchführungsverantwortung).

In dem Feld „Personaleinsatz" stellen sich an das Personalmanagement der sozialen Verantwortung Anforderungen in zweierlei Hinsicht:

- Dem Mitarbeiter sollten nur solche Aufgaben zur Erledigung übertragen werden, deren Beanspruchung diese ergibt sich aus den Arbeitsinhalten, den Arbeitszeiten und dem Arbeitsplatz (unter Berücksichtigung technischer und organisatorischer Aspekte) - von diesem mittels seiner Eigenschaften und Fähigkeiten bewältigt werden kann. Bei der Beurteilung, ob dieses Ziel des Personaleinsatzmanagements erreicht worden ist, wird danach gefragt, ob die Arbeit für den Mitarbeiter zufrieden stellend, zumutbar, erträglich und ausführbar (in dieser Rangfolge) ist; dabei spielt die altersspezifische Bewertung des Arbeitsplatzes angesichts der demografischen Entwicklung der Krankenhaus-Belegschaften eine zentrale Rolle.

- Die Gestaltung des Personaleinsatzes stellt in einem Unternehmen, das seine Leistungen „rund-um-dieUhr" anbieten muss und damit Mitarbeiter "rundum-die-Uhr" anwesend sein müssen, besonders hohe Anforderungen sozialer Art. So kann unter anderem erwartet werden, dass

- schon die durch die Geschäftsführung erlassenen Grundsätze für die Gestaltung der Dienstpläne ausreichend Spielräume vorsehen, damit bei der Festlegung der leistungsbereichs-individuellen Dienstpläne die personellen und organisatorischen $\mathrm{Be}$ sonderheiten der Leistungsbereiche, aber auch die Interessen anderer Stakeholder berücksichtigt werden können;

- sowohl die Dienstplangrundsätze als auch die leistungsbereichs-individuellen Dienstpläne auf der Grundlage eines argumentativen Dialogs entwickelt werden und begründbar sind und dass

- die Dienstpläne Ergebnis eines fairen Ausgleichs zwischen den Unternehmensinteressen und den Bedürfnissen der Führungskräfte und Mitarbeiter sind.

Die Zuweisung einer Aufgabe an eine Führungskraft und/ oder einen Mitarbeiter sollte das Ergebnis einer argumentativen, dialogischen Verständigung zwischen der Geschäftsführung/Führungskraft und der/dem Führungskraft/Mitarbeiter und von der jeweils zuweisenden Instanz mit guten Argumenten begründet werden können.

\section{Personalkostenmanagement}

Das Personalkostenmanagement sucht eine Antwort nicht nur auf die Frage nach einer die Existenz des Krankenhauses sichernden Personalkostenplanung. Die Beantwortung dieser Frage ist aus der Sicht des Managements der sozia- len Verantwortung zwar auch von Bedeutung, soll aber aus Platzgründen hier nicht vertieft werden. Ein weiteres Thema des Personalkostenmanagement ist die Suche nach einer Antwort auf die Frage nach der Angemessenheit der Honorierung der Führungskräfte und der Mitarbeiter.

Dabei werden zwei Wirkungen der Honorierung in den Blick genommen, nämlich die Finanzierungswirkung und die Steuerungswirkung. So dient die Honorierung einerseits der Finanzierung eines auskömmlichen Lebensunterhaltes der Führungskräfte und Mitarbeiter und leistet damit einen ersten, meist allerdings begrenzten Beitrag zur Befriedigung eines Mitarbeiterbedürfnisses und damit zur Motivation der Führungskräfte und Mitarbeiter zu besonderen Anstrengungen. Ihre eigentliche Steuerungswirkung entfaltet die Honorierung erst dann, wenn die Geschäftsführung mit ihr zum Ausdruck bringt, welche Leistungen und welches Verhalten sie belohnen will. Letzteres gilt insbesondere für Handlungen, die im Sinne des Managements der sozialen Verantwortung erlaubt/erwünscht bzw. verboten/unerwünscht sind; sozial verantwortliches Handeln soll sich für die Verantwortungsträger finanziell lohnen.

Die Honorierung befriedigt - wenn sie entsprechend ausgestaltet wird - eine ganze Reihe weiterer Bedürfnisse der Führungskräfte und Mitarbeiter und leistet damit weitere Beiträge zu deren Motivation:

- Sie soll deren Bedürfnis nach Sicherheit befriedigen. Dazu gehört, dass das Gehalt oder der Lohn nicht nur ausreichend hoch für die Finanzierung des Lebensunterhaltes ist - das wurde weiter oben schon erwähnt. Die Führungskräfte und die Mitarbeiter erwarten darüber hinaus, dass die Höhe des vereinbarten Entgelts nicht im Falle einer finanziellen Notlage des Krankenhauses nach unten korrigiert wird (s. zum Beispiel Vivantes, S. 25).

- Die Honorierung soll "gerecht" sein. Das Gehalt, das die Führungskräfte und die Mitarbeiter erhalten, soll der sich aus der Erledigung einer Aufgabe ergebenden Belastung und der Leistung, die die Führungskräfte und Mitarbeiter erbringen, entsprechen.

- Nicht selten ist die Höhe der Honorierung der einzige Indikator, mit dessen Hilfe die Führungskräfte und die Mitarbeiter das Ausmaß an Wertschätzung, die ihnen von der Geschäftsführung gezollt wird, feststellen können.

Die Honorierung der Akteure in einem Krankenhaus ist in Tarifverträgen geregelt oder das Ergebnis einer individuellen Vertragsgestaltung. Die Tarifverträge sind das Ergebnis einer argumentativen, dialogischen Verständigung zwischen den Tarifparteien; sie sind ein Kompromiss zwischen den Bedürfnissen der Führungskräfte und Mitarbeiter auf der einen Seite und denen vor allem der Krankenkassen und der Eigentümer. Den individuellen Verträgen liegen 
nicht selten Muster-Verträge zugrunde ${ }^{53}$, die nur bedingt als Ergebnis einer argumentativen, dialogischen Verständigung qualifiziert werden können, die aber genügend Spielraum für die individuelle Ausgestaltung lassen.

Die Tarifverträge lassen im Regelfall einen gewissen Spielraum für eine leistungs-/erfolgsorientierte Bezahlung zu. Die konkrete Honorierung ist dann wie auch der Inhalt des individuellen Vertrages Ergebnis einer argumentativen, dialogischen Verständigung zwischen dem Mitarbeiter und seinem Arbeitgeber. Die Gewichte zwischen Arbeitgeber und Arbeitnehmer sind - je nach Arbeitsmarktsituation nicht immer gleich verteilt. Deshalb kommt es darauf an, dass sich der Tarifvertrag und die daraus abgeleitete Honorierung eines Arbeitnehmers und das Ergebnis der individuellen Vertragsgestaltung mit guten Gründen verteidigen und gegenüber dem Vertragspartner begründen lassen.

\section{Personalführung}

Von zentraler Bedeutung für die Sicherung einer dauerhaften Orientierung des Handelns in Krankenhäusern an den Grundideen des Managements der sozialen Verantwortung ist die Personalführung. Sie ist auf das Beeinflussen des Verhaltens der Verantwortungsträger ausgerichtet. Sie fördert auf diesem Weg die Sensibilisierung der Verantwortungsträger für sozial verantwortliches Handeln und deren Bereitschaft, sich mit dem neuen ManagementKonzept zu identifizieren und entsprechend den einschlägigen Normen handeln zu wollen. Insofern befriedigt die verantwortungsvolle Ausübung der Mitarbeiter-Führung nicht nur Mitarbeiter-Bedürfnisse; verantwortungsvolle Mitarbeiter-Führung trägt mittelbar dazu bei, dass auch die Bedürfnisse anderer Stakeholder bei Entscheidungen der Adressaten der Personalführung im Sinne der Goldenen Regel berücksichtigt werden.

Anforderungen an die Personalführung stellen sich zwecks Realisierung der Grundpostulate des Managements der sozialen Verantwortung in vier Dimensionen eines Führungskonzepts:

Erstens ergibt sich die Notwendigkeit einer Personalführung im Sinne des Managements der sozialen Verantwortung aus dem Umstand, dass die Führungskraft ihre Mitarbeiter immer noch nicht selten als „Produktionsfaktor Arbeit" sieht, der zwecks Erreichen der Unternehmensziele instrumentalisiert wird. Die Mitarbeiter dagegen haben eigene Bedürfnisse, deren Befriedigung mit dem Verfolgen der Unternehmensziele und der Ziele der Führungskraft sowie mit der Befriedigung anderer Stakeholder-Bedürfnisse in Einklang gebracht werden müssen.

53 Siehe zum Beispiel die Beratungs- und Formulierungshilfe Chefarztvertrag, herausgegeben durch die Deutsche Krankenhausgesellschaft e.v.
Zu fordern ist deshalb, dass ein Führungskonzept praktiziert wird, das

- Leitungsaufgaben einschließlich der Befugnis, Leitungsentscheidungen zu fällen und das Ergebnis anderen zur Ausführung anzuweisen, möglichst weitgehend den unteren Leitungsebenen zuordnet,

- einen - wo immer dieses möglich ist - als partizipativ bezeichneten Führungsstil vorsieht, bei dem der Entscheidungsprozess durch Mitarbeiter geprägt wird (Tannenbaum; Schmidt, S. 96), und das

- von den Führungskräften verlangt, Anweisungen nicht mehr nur aus der Positionsautorität abzuleiten, sondern argumentativ zu begründen (Wittmann, S. 192).

Zweitens ist als Basis der für Krankenhäuser spezifischen Teamarbeit (Wahren, S. 164) zu fordern:

- die Förderung eines offenen, vertrauensbasierten und respektvollen Klimas,

- das Fordern und das Fördern des auf Respekt beruhenden Umgangs der Berufsgruppen miteinander,

- das Bewusstmachen und der Abbau von Spannungen, die durch soziale Konflikte entstehen,

- der Schutz der Gruppenmitglieder vor persönlichen Angriffen,

- die Förderung einer ausgewogenen Kommunikation,

- die Überwachung der Einhaltung von Vertrauens- und Kommunikationsregeln und

- der Einbezug von Außenseitern.

Drittens ist darüber hinaus zu fordern, dass Führungsinstrumente eingesetzt werden, die geeignet sind,

- die Bedürfnisse der Mitarbeiter nach Sicherheit, Wertschätzung, Selbstverwirklichung und Selbstentfaltung (Staehle, S. 853) zu befriedigen (= Führen mit Zielvereinbarungen) und

- ein Führungsverständnis zu fördern, das auf eine andere Art der Autoritätsausübung durch die Führungskräfte angelegt ist - diese vereinbaren Ziele, richten Kommunikationsstrukturen ein, strukturieren Kommunikationsprozesse, kommentieren die Qualität der Arbeit, initiieren Veränderungen der Prozesse und managen Konflikte (= Mitarbeiterorientierungsgespräch) (Grossmann; Zepke, S. 84ff.).

Für das Zusammenwirken einer großen Zahl von Menschen (Führungskräfte und Mitarbeiter) in einem multipersonal und arbeitsteilig organisierten Krankenhaus bedarf es viertens spezifischer, auf die Befriedigung der Bedürfnisse der Patienten und der Mitarbeiter ausgerichteter Führungsgrundsätze (Naegler et al., S. 275 und die dort genannte Literatur; Kreikebaum, S. 25off.), wie zum Beispiel:

- Sozial verantwortetes Führen fördert die Auseinandersetzung mit dem Spannungsfeld zwischen subjektivem Wohlbefinden und öffentlicher Wohlfahrt.

- Sozial verantwortetes Führen fordert die Auseinandersetzung mit der Begrenztheit der Ressourcen. 
- Sozial verantwortetes Führen respektiert, dass Mitarbeiter
- loyal gegenüber Patienten sind und
- im Interesse der Patienten handeln.

- Sozial verantwortetes Führen akzeptiert, dass Gesundheit ein individuelles Gut ist und dass es der Beitrag des Krankenhauses ist, dem Patienten die Möglichkeit zur Ausübung seiner Autonomie zu erhalten oder wieder herzustellen ${ }^{54}$ (Wallner, S. 93f.).

- Mit dem Recht, über die Arbeitskraft zu verfügen, erwirbt der Unternehmer in keiner Weise auch das Recht, im Rahmen bestehender Gesetze oder Verträge beliebig über sie zu verfügen.

- Der Führungsinteraktion liegt personale Autorität zugrunde.

- Die Menschenwürde ist zu respektieren.

- Der Mitarbeiter hat Anspruch auf Solidarität.

- Was auch immer erkannt wird, wird auf die Weise des Erkennenden erkannt.

- Sozial verantwortetes Führen

- impliziert das Erkennen und Vermeiden von Kommunikationsstörungen,

- geschieht in prinzipiell reversibler Kommunikation, - akzeptiert das Gewissensurteil des Geführten,

- begünstigt die Identifikation mit dem Unternehmen,

- respektiert fremde Würde und Freiheit und

- setzt entwickelte Konfliktfähigkeit des Führenden voraus.

Wenn Personalführung auf die skizzierte Weise praktiziert wird, werden mehrere der Grundpostulate eingehalten: Es werden die Interessen der betroffenen Mitarbeiter berücksichtigt. Die Führung erfolgt auf der Grundlage einer argumentativen, dialogischen Verständigung. Die Mündigkeit der Mitarbeiter wird gefördert. Moralische Prinzipien werden beachtet. Die Führungskraft begründet ihre Führungsmaßnahmen.

\subsubsection{Die Organisation der Leitungstätigkeit}

\section{Einführung}

Das Krankenhaus bedarf einer Unternehmensorganisation, die sozial verantwortliches Handeln fördert. Organisationale Barrieren, die der Wahrnehmung von Verantwortung im Wege ste-

54 Wenn Gesundheit als öffentliches Gut verstanden wird, dann ist es die Aufgabe des Gesundheitswesens dazu beizutragen, dass eine der Eigenschaften des volkswirtschaftlichen Produktionsfaktors „Humankapital“ - nämlich die Gesundheit - als wichtiger Wettbewerbsfaktor der Gesellschaft im Wirtschaftsprozess gesichert, gesteigert und/oder wieder hergestellt werden kann. hen, werden abgebaut (Cöbel 2006, S. 234); deren Entstehen wird verhindert. Von zentraler Bedeutung, weil sie der Rahmen ist für das Realisieren des Controllings und der Personalarbeit, ist in diesem Zusammenhang die Organisation der Leitungstätigkeit.

In diesem Zusammenhang sind drei Gestaltungs-Bereiche zu unterscheiden (s. Abb. 35):

1. Als Erstes stellt sich die Frage, wie die aufbauorganisatorische Grundstruktur des Krankenhauses beschaffen sein muss. Diese ist der Rahmen für das Realisieren der Leitungstätigkeit und stellt den Spielraum für sozial verantwortliches Handeln zur Verfügung.

2. Der Gegenstand der weiteren Überlegungen ist die cliederung des Krankenhauses in Leitungsebenen. Oder anders gefragt: Werden die Aufgaben der Leitung des Krankenhauses vernünftigerweise mehr zentral oder mehr dezentral wahrgenommen? Dieser Frage liegt die Annahme zugrunde, dass flachere Hierarchien sowie dezentrale und überschaubare Leistungsbereiche als Ergebnis der Dezentralisierung der Leitung des Krankenhauses die Wahrnehmung von Verantwortung fördern.

3. Sodann ist zu überlegen, mit wie viel Personen mit welcher Qualifikation die Instanzen, denen die Wahrnehmung der Managementfunktionen übertragen wird, besetzt werden und wie - wenn es mehrere Personen sind die Zusammenarbeit dieser Personen sowie die Zurechnung und Wahrnehmung von Verantwortung erfolgen. In diesem Zusammenhang stellt sich die Frage, welchen Einfluss die verschiedenen Gestaltungsoptionen auf die Entscheidungs- und Verantwortungsbereiche der Menschen haben, denen Leitungsaufgaben übertragen worden sind.

\section{Die aufbauorganisatorische Grundstruktur des Krankenhauses}

\section{a) Charakterisierung}

Wenn bisher von Organisation die Rede war, dann war damit die Arbeitsorganisation als Mikro-Struktur des Krankenhauses gemeint. Diese ist im Regelfall so flexibel gestaltet, dass sie als (proaktive) Reaktion auf Veränderungen der Krankenhaus-Umwelt schnell geändert werden kann. Die Makro-Struktur des Unternehmens, dessen aufbauorganisatorische Grundstruktur, ist dagegen für einen Bestand über einen längeren Zeitraum 
konzipiert - gegebenenfalls auch für die gesamte Zeit der Unternehmens-Existenz.

Ausgehend von den beiden Aufgabenmerkmalen „Verrichtung“ und „Objekt“ werden zwei Grundmodelle der aufbauorganisatorischen Grundstruktur des Krankenhauses, das Verrichtungsmodell und das Objektmodell, unterschieden (Staehle, S. 739f.) (s. Abb. 40, oben und unten); in der Literatur, aber auch in der Praxis genießt in der jüngsten Vergangenheit zusätzlich das Prozessmodell immer größere Aufmerksamkeit (Staehle, S. 749) (s. Abb. 42).

$\mathrm{Zu}$ der Strukturierung eines Unternehmens nach dem Verrichtungsmodell (s. Abb. 40, oben) entschließt man sich, weil von der funktionalen Spezialisierung der Mitarbeiter ein hohes Maß an Qualität und Effizienz der Leistungserstellung erwartet wird. In den Kernbereichen der Krankernhäuser unterscheidet man entsprechend diesem Gliederungsmodell die Aufnahme der Patienten,
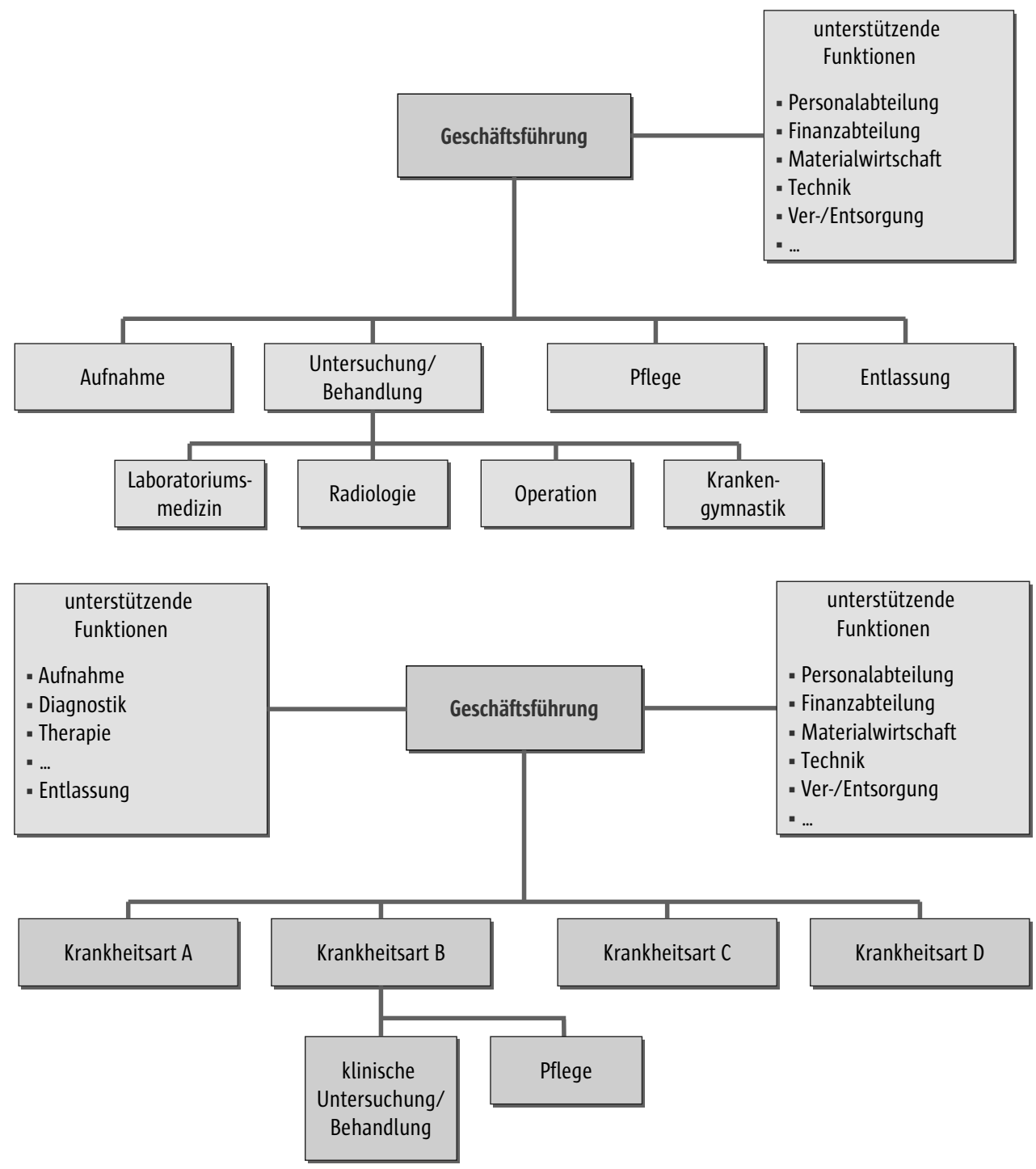

Abb. 40 Grundstruktur des Krankenhauses - Verrichtungsmodell (oben) und Objektmodell (unten) 
den Untersuchungs- und Behandlungsbereich, den Pflegebereich und die Entlassung. Bei weiterer Differenzierung zum Beispiel des Untersuchungs- und Behandlungsbereichs, entstehen in der nächsten Ebene Leistungsbereiche der medizinischen Infrastruktur wie die Laboratoriumsmedizin und die Radiologie. Cliedert man den Verwaltungs-, Versorgungs-/Entsorgungs- und Technikbereich entsprechend dem Verrichtungsmodell, dann entstehen Leistungsbereiche der nicht-medizinischen Infrastruktur wie die Personal- und die Finanzabteilung.

Das Objektmodell (s. Abb. 4o, unten) wird in Unternehmen mit einem heterogenen Produktionsprogramm bevorzugt, weil man sich von der auf Produkte und/oder Kunden bezogenen Spezialisierung der Mitarbeiter eine hohe Qualität und Effizienz der Leistungserstellung verspricht. Wegen des für Krankenhäuser charakteristischen heterogenen Produktionsprogramms und wegen der auf Krankheitsbilder erfolgten Spezialisierung vor allem der Ärzte sind die Krankenhäuser - was die klinischen Leistungsbereiche anbelangt - herkömmlicherweise in der Leitungsebene, die der Geschäftsführung unmittelbar nachgeordnet ist, anhand des Objektmodells organisiert. In der dann folgenden Leitungsebene und für die diversen unterstützenden Funktionen des medizinischen und nicht-medizinischen Bereichs kommt meist das Verrichtungsmodell zum Zuge.
Die Entwicklung der Medizin und der MedizinTechnik und die daraus folgende Spezialisierung von Ärzten, Krankenpflegekräften und Vertretern diverser paramedizinischer Berufsgruppen haben dazu geführt, dass klinische Leistungsbereiche immer mehr in Sub-Bereiche aufgegliedert worden sind und werden (s. Beispiel 1 und Abb. 41). Die Grundstruktur des Krankenhauses wird immer komplizierter. Es werden Leistungsbereiche geschaffen, die eine kleinere Zahl von Patienten zu behandeln haben und die mit einer immer kleineren Zahl von hochspezialisierten Mitarbeitern ausgestattet sind. Insbesondere die Behandlung multimorbider Patienten kann schon bei der einfachen objektorientierten Gliederung des Krankenhauses zu einem Problem werden (In welche Fach-Abteilung werden die Patienten aufgenommen und wer ist für deren Behandlung verantwortlich?). Die Erweiterung der Organisationsstruktur in Form von Sub-Bereichen verstärkt die Nachteile einer horizontalen Arbeitsteilung.

\section{Beispiel 1 (s. auch Abb. 41)}

Das medizinische Fachgebiet "Innere Medizin" wird zunehmend nicht mehr nur durch eine Fachabteilung „Innere Medizin" vertreten. Die Spezialisierung, die innerhalb dieses Fachgebietes stattgefunden hat, hat dazu geführt, dass auch schon in mittelgroßen Krankenhäusern das Fachgebiet „Inne-

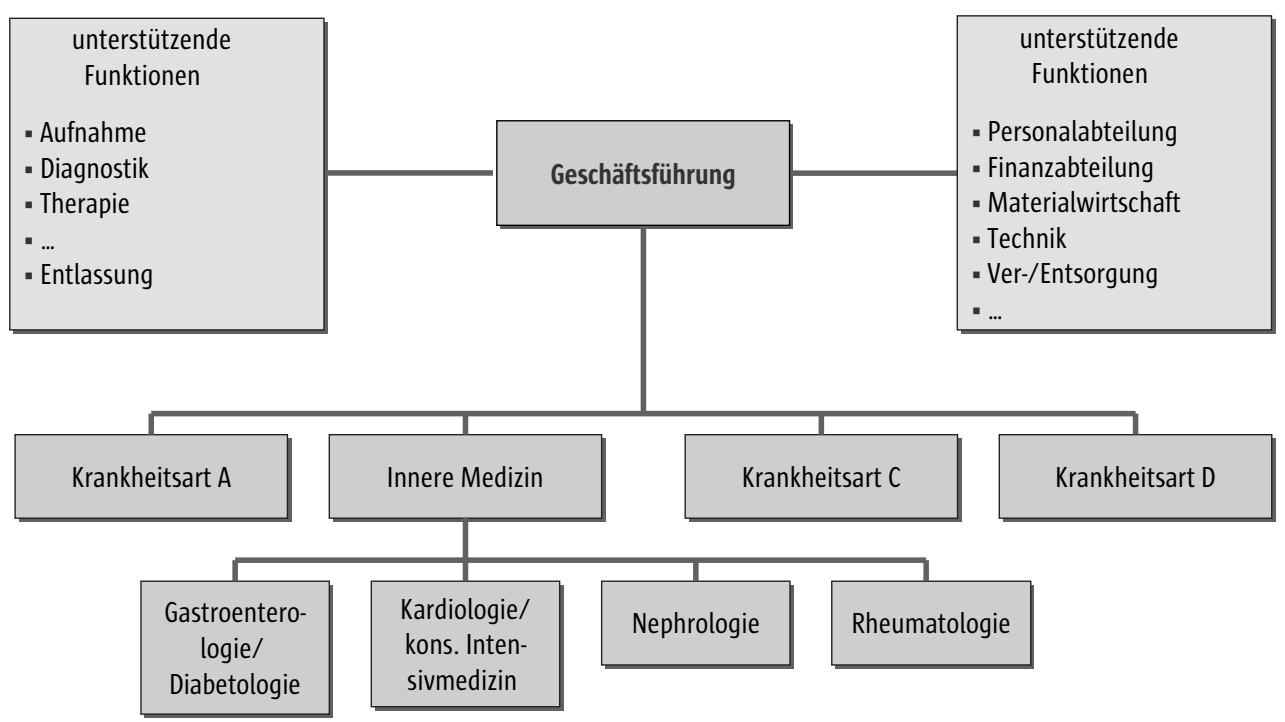

Abb. 41 Grundstruktur des Krankenhauses - Objektmodell - erweitert 
re Medizin“ durch mehrere Fachabteilungen - wie zum Beispiel durch die Fachabteilungen „Gastroenterologie und Diabetologie“, „Kardiologie und konservative Intensivmedizin“ sowie „Nephrologie“ und „Rheumatologie“- angeboten wird.

$\mathrm{Zu}$ der inneren Struktur der Leistungsbereiche: Immer häufiger gehen Krankenhäuser dazu über, die Leistungsbereiche - ob nach dem Verrichtungs- oder nach dem Objektmodell entstanden - als „Profit-Center“ einzurichten und diesen eine eigene Ergebnisverantwortung zuzuweisen $^{55}$. Der Leiter eines solchen Leistungsbereichs ist nicht mehr nur für die Patientenbehandlung, sondern auch für das wirtschaftliche Ergebnis seiner Abteilung verantwortlich; er fällt alle dafür einschlägigen Entscheidungen und setzt diese um. Letzteres gilt vor allem für die klinischen Leistungsbereiche (deshalb ist im Rahmen der weiteren Untersuchung nur noch von diesen die Rede).

Einerseits konnte die Qualität der Behandlung in Folge des durch die Spezialisierung ermöglich-

55 Allerdings fehlt diesen Profit-Centren zumindest vorläufig noch ein zentrales Merkmal: Es ist innen nicht erlaubt, Leistungen, die sie von einem anderen Leistungsbereich des Krankenhauses beziehen können - wie zum Beispiel Leistungen eines Zentrallaboratoriums -, von einem externen Anbieter einzukaufen, weil diese dort zu geringeren Kosten zu erhalten sind. ten Kompetenzzuwachses kontinuierlich verbessert werden. Andererseits hat die Zahl der Schnittstellen zwischen den verschiedenen Leistungsbereichen des Krankenhauses zugenommen. Die Koordination der Behandlung insbesondere die der zunehmenden Zahl multimorbider Patienten wird erschwert - mit negativen Auswirkungen auf die Behandlungsqualität und -effizienz. Hinzu kommt: Die als Konsequenz der Spezialisierung erfolgte Aufteilung vieler Fachabteilungen in Sub-Leistungsbereiche hat dazu geführt, dass die Zahl der Mitarbeiter in den Sub-Leistungsbereichen geringer geworden ist und dass deshalb die Patienten immer häufiger von wechselnden Personen betreut werden; das für die Behandlung notwendige Vertrauensverhältnis zwischen den Patienten und den Mitgliedern des Behandlungsteams leidet.

Die zuletzt skizzierte Charakterisierung der aufbauorganisatorischen Grundstruktur des Krankenhauses und deren Folgen hat die Entwicklung neuer Gestaltungskonzepte begünstigt. Die an dem Verrichtungsmodell orientierte Grundstruktur wird zunehmend durch eine an dem Prozessmodell ausgerichtete überlagert (s. Abb. 42). Die Grundstruktur des Krankenhauses wird vom Behandlungsergebnis, also von dem Nutzen der Behandlung für den Patienten her gedacht - von dem Produkt ausgehend, das durch das Kranken-

\section{Kernprozesse}

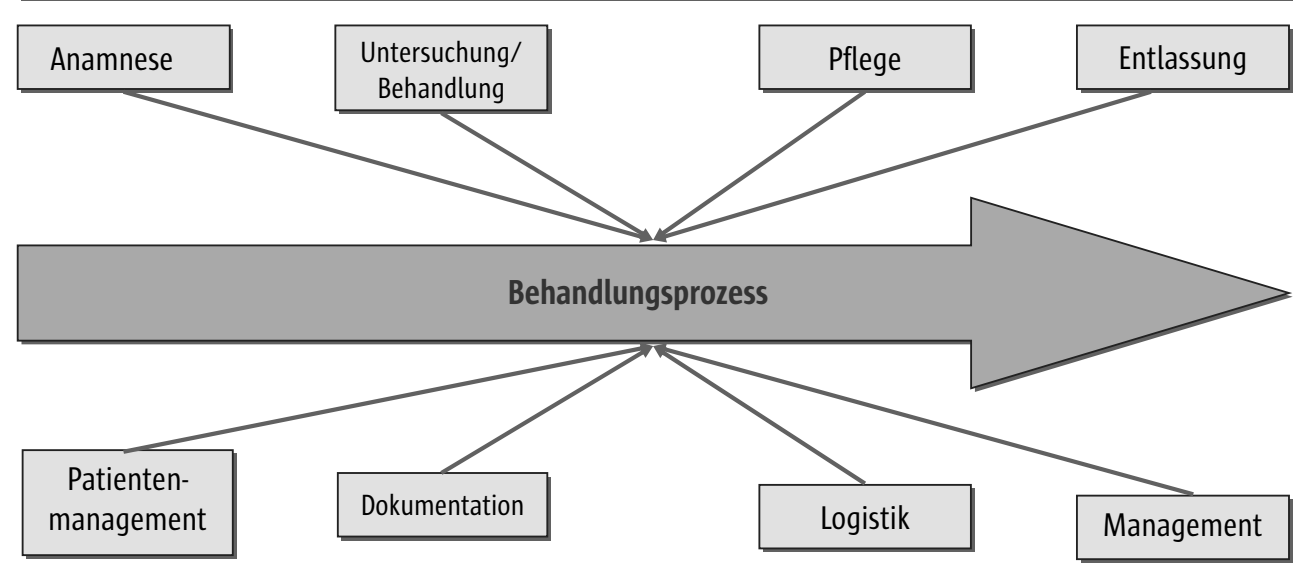

unterstützende Prozesse (unter anderem)

Abb. 42 Grundstruktur des Krankenhauses - Prozessmodell 
haus angeboten wird. Alle Prozesse und Aktivitäten werden so konzipiert, dass sie einen maximalen Beitrag zur Realisierung des Behandlungsziels leisten können. Unnütze Maßnahmen werden vermieden.

Dazu gehört es, dass es einen für den Behandlungsprozess und damit auch für einen konkreten Patienten verantwortlichen Mitarbeiter (der Casemanager) gibt. Dieser sorgt dafür, dass die TeilProzesse, die einen Beitrag zur Realisierung des Behandlungszieles leisten, von den dafür zuständigen Leistungsbereichen sach- und zeitgerecht realisiert und als Teil-Prozesse in den Behandlungsprozess integriert werden. Der Casemanager hat damit ein hohes Maß an Verantwortung. Im Regelfall deckt sich aber der ihm zugewiesene Entscheidungsspielraum bei weitem nicht mit seinem Verantwortungsbereich. Seine Möglichkeiten, Bedürfnisse der Patienten bei seinen Entscheidungen angemessen zu berücksichtigen, sind damit eher begrenzt.

Ein bestimmendes Merkmal des Prozessmodells - und das ist ein weiterer grundlegender Unterschied zu einem reinen Verrichtungsmodell - ist die Verknüpfung der Teil-Prozesse im Sinne einer Kunden-Lieferanten-Beziehung. Jeder Teilprozess wird abgeschlossen mit einem Ergebnis, über das sich vor dem Start des Teil-Prozesses der Lieferant und der Kunde in zeitlicher und inhaltlicher Hinsicht verständigt hatten (s. Beispiel 2) und das als Input für den nächsten Teilprozess benötigt wird. Die Betrachtung der Schnittstellen zwischen den Arbeitsabläufen erhält ein sehr viel größeres Gewicht. Dem Behandlungsprozess wohnt ein sehr viel höheres Maß an Selbstkontrolle inne (Eichhorn, S. 163).

\section{Beispiel 2}

Bevor ein Patient geröntgt werden kann, legt der für die Therapie zuständige Arzt fest, welche Befunde er benötigt, um die Diagnose als Grundlage für die Therapieplanung, diese wiederum basiert auf einem konkreten Behandlungsziel, erarbeiten zu können. Er teilt dieses dem Radiologen mit, der daraufhin die radiologische Untersuchung planen und durchführen kann. Vorher prüft der Radiologe, ob der Auftrag, den er erhalten hat, hinreichend genau beschrieben ist und fordert gegebenenfalls eine Ergänzung/ Präzisierung an.

Der für die Therapie zuständige Arzt erhält als Ergebnis des Teil-Prozesses „Röntgen“ den Befund und prüft, ob die darin enthaltenen Aussagen geeignet sind, eine valide
Diagnose zu erarbeiten. Gegebenenfalls bittet er den Radiologen, seine Aussagen zu präzisieren, was möglicherweise die Wiederholung der radiologischen Untersuchung notwendig macht.

Die skizzierte Kunden- (= der für die Therapie zuständige Arzt)-Lieferanten- (= der Radiologe)-Beziehung ist in einer zunehmenden Zahl von Krankenhäusern Gegenstand eines Geplanten Behandlungspfades. Dieser ist eine multiprofessionelle Arbeitsanweisung (Tecklenburg, S. 303), die den gesamten medizinischen Behandlungsprozess also nicht nur die stationäre Behandlung - umfasst. Sie gilt nicht nur für den ärztlichen Bereich, sondern auch für alle anderen an der Patientenbehandlung beteiligten Berufsgruppen.

Zusammenfassend kann festgehalten werden:

Die Grundstruktur eines Krankenhauses ist gekennzeichnet durch Ziel- und Interessendivergenzen der an dem Behandlungsprozess beteiligten und - dadurch bedingt - durch einen hohen Bedarf an Koordination, um die Handlungen der Verantwortungsträger auf die Realisierung der Unternehmensziele (Staehle, S. 528) und auf die Befriedigung der diversen Stakeholder-Bedürfnisse ausrichten zu können. Dieser Befund gilt unabhängig davon, ob die Grundstruktur eine Kombination aus einem Objektmodell (Ebene der klinischen Abteilungen unterhalb der Geschäftsführung) mit einem Verrichtungsmodell (Ebene unterhalb der klinischen Abteilungen sowie die medizinischen und nicht-medizinischen SupportAbteilungen) oder ob sie an dem prozessorientiertes Modell ausgerichtet ist.

Der Koordinationsbedarf wird verstärkt durch die skizzierte und weiter zunehmende Spezialisierung der Ärzte, der Pflegekräfte und der Vertreter paramedizinischer Berufsgruppen und durch die daraus resultierenden Konsequenzen für die Abteilungsbildung. Eine weiterhin zunehmende Zahl der Grenzen und der Schnittstellen zwischen den verschiedenen Leistungsbereichen des Krankenhauses und der Unterbrechungen der Behandlungs-, Support- und Betriebsführungsprozesse sind die Folge bei starken Abhängigkeiten zwischen den Abteilungen (Staehle, S. 529).

\section{b) Anforderungen an die Gestaltung}

Die organisationalen Barrieren für das Wahrnehmen von Verantwortung sind also hoch. Sie können mit den Mitteln, wie sie für die Abteilungsorganisation beschrieben worden sind, 
nicht überwunden werden - zum Beispiel durch die Zusammenfassung einzelner Funktionen zu einem ganzheitlichen Arbeitsprozess; die Vorteile, die durch Spezialisierung erzielt werden können, würden damit aufgegeben werden.

Was also bleibt für die Lösung des skizzierten Problems? Zwei Gestaltungs-Felder bieten sich an (Mintzberg, S. 142ff.; Türk, S. 46 und 127) (s. folgenden Textabschnitt):

1. Standardisierung der Prozesse und

2. Rekrutierung, Aus- und Weiterentwicklung sowie Einsatz der Mitarbeiter

\section{Anforderungen an die aufbauorganisatorische Grundstruktur}

\section{Gestaltungs-Felder, Anforderungen an die Gestaltung}

\section{Standardisierung der Prozesse}

Die Reduzierung des Koordinationsaufwandes mit dem Ziel einer besseren Integration der an der Behandlung eines Patienten beteiligten Abteilungen und damit einer Verbesserungen der Voraussetzungen für das Wahrnehmen von Verantwortung im Sinne der Goldenen Regel kann unter anderem dadurch erreicht werden, dass die Behandlungsund Supportprozesse mittels Behandlungsempfehlungen, - leitlinien oder -richtlinien standardisiert werden. In den Standards werden jene Maßnahmen festgelegt, die zum Erreichen des Behandlungsziels und unter Berücksichtigung der aktuellen Befindlichkeit des Patienten notwendig sind.

Die Standardisierung der Prozesse und deren Anwendung dienen der Befriedigung diverser Bedürfnisse der Patienten (unter anderem nach Sicherheit und Qualität der Behandlung), der Mitarbeiter (unter anderem nach Sicherheit im Sinne des Bewältigenkönnens der innen übertragenen Aufgaben und nach dem Ausfüllen ihrer Agenten-Rolle) sowie der Eigentümer und Krankenkassen (unter anderem nach der Steigerung der Qualität und der Effizienz der Patienten-Behandlung).

Dieses System setzt voraus, dass der Behandlungsprozess in zeitlicher Hinsicht durch eine argumentative, dialogische Verständigung unter allen jenen organisiert wird, die durch Teilleistungen zu der Realisierung des Behandlungszieles beitragen.

\section{Rekrutierung, Aus- und Weiterentwicklung sowie Einsatz der Mitarbeiter}

Die Überwindung der skizzierten organisationalen Barrieren kann mit Führungskräften und Mitarbeitern gelingen, die sozial verantwortliches Handeln praktizieren wollen und auch können. Deshalb ist es wichtig, schon bei der Einstellung von Mitarbeitern darauf zu achten, dass diese sich mit dem Konzept des Managements der sozialen Ver- antwortung identifizieren und erkennen lassen, dass sie dieses in der Praxis umsetzen wollen.

Vorhandene Mitarbeiter, deren Fähigkeiten, die für die Wahrnehmung von Verantwortung essentiell sind, nicht ausreichend ausgeprägt sind, werden durch Aus- und Weiterbildung in die Lage versetzt, soziales Handeln erfolgreich zu realisieren.

\section{Zentralisation versus Dezentralisation von Leitungsaufgaben}

\section{a) Charakterisierung}

Die Begriffe Zentralisation sowie Dezentralisation werden in der Literatur und in der Praxis unterschiedlich interpretiert. In der vorliegenden Untersuchung werden diese Begriffe wie folgt verwendet:

Leitungsaufgaben können - in vertikaler Richtung verteilt - an der Spitze des Unternehmens zusammengefasst (= Zentralisierung) oder möglichst weitgehend den Leitungsebenen an der Basis des Krankenhauses zugeordnet werden (= Dezentralisierung). Kriterien für die Verteilung der Leitungsaufgaben auf die Leitungsebenen sind unter anderem

- deren Bedeutung für das Realisieren der Unternehmensziele,

- die Art und die Stärke des Einflusses, den die von den Leitungsentscheidungen Betroffenen auf die Durchsetzung ihrer Interessen ausüben können, und deren Verortung auf einer der drei Allokationsebenen,

- die Wichtigkeit der von den Stakeholdern zu erwartenden Beiträge und

- die Qualität des Kontaktes der Verantwortungsträger zu den Adressaten der Verantwortung.

Das richtige Maß an Zentralisierung bzw. Dezentralisierung von Leitungsaufgaben $z u$ finden ist ein kompliziertes und nicht selten ideologisch und emotional besetztes Unterfangen.

\section{b) Anforderungen an die Gestaltung}

Mit der kurzen Charakterisierung der Leitungstätigkeit und der Nennung der Kriterien, anhand derer über die Verteilung der Leitungsaufgaben auf Leitungsebenen entschieden wird, ist das Feld identifiziert, innerhalb dessen Maßnahmen so gestaltet werden sollten, dass organisationale Barrieren für das Wahrnehmen von Verantwortung abgebaut werden bzw. nicht entstehen. 


\section{Anforderungen an die Gestaltung der Verteilung von Leitungsaufgaben}

\section{Gestaltungs-Feld, Anforderungen an die Gestaltung}

\section{Leitungsaufgaben dort lokalisieren, wo die für bestimmte Stakeholder relevanten Entscheidungen ... vernünftigerweise gefällt werden}

Entscheidungen, bei denen die Anliegen des KrankenhausEigentümers und die der Krankenkassen zu berücksichtigen sind, werden vernünftigerweise der Geschäftsführung zugewiesen - also im Sinne der Zentralisierung der Leitungstätigkeit in der Spitze des Unternehmens zusammengefasst. Der enge Kontakt zwischen den Mitgliedern der Geschäftsführung und dem Eigentümer bzw. den Vertretern der Krankenkassen versetzt die Geschäftsführung am ehesten in die Lage, die Anliegen der genannten Geschäftsführungs-Verantwortungs-Adressaten - auch unter Berücksichtigung der Interessen des Krankenhauses - als Ergebnis eines Dialogs mit den Betroffenen sachgerecht zu bewerten und zu gewichten. Die Geschäftsführung ist damit am besten im Stande, Entscheidungen, bei denen Anliegen des Eigentümers und der Krankenkassen eine Rolle spielen, im Sinne der Goldenen Regel zu fällen.

Der Einfluss der genannten Stakeholder auf das betriebliche Geschehen ist rechtlich, vertraglich und finanziell begründet; sie sind in der Meso- bzw. Makroebene verortet. Deren Einfluss auf das betriebliche Geschehen ist deshalb besonders stark. Die von diesen Stakeholdern zu erwartenden Beiträge für das Krankenhaus sind von großer Wichtigkeit. Auch diese Gründe sprechen dafür, Entscheidungen, bei denen Anliegen des Eigentümers und der Krankenkassen zu berücksichtigen sind, der Geschäftsführung zuzuweisen.

Die Geschäftsführung wird auf Grund ihrer genauen Kenntnis der Eigentümer- und Krankenkassen-Bedürfnisse den Verantwortungsträgern in den ihr nachgeordneten Leitungsebenen vorgeben, mit welchem Gewicht die Eigentümer- und Krankenkassen-Bedürfnisse bei deren Entscheidungen zu berücksichtigen sind. So wird sichergestellt, dass die genannten Anliegen bei Entscheidungen auch in allen anderen Leitungsebenen angemessen berücksichtigt werden können.

Entscheidungen, bei denen die Anliegen der Gesamtheit der Patienten, der einweisenden Ärzte, der Führungskräfte und der Mitarbeiter berücksichtigt werden sollen, werden zweckmäßigerweise der Geschäftsführung zugewiesen. Als Ergebnis einer dialogischen Verständigung mit den Betroffenen und/oder deren Agenten werden deren Anliegen bewertet, gewichtet und im Rahmen von Grundsatz-Entscheidungen berücksichtigt und als Rahmen für die Entscheidungen anderer Verantwortungsträger verbindlich gemacht.
Entscheidungen, bei denen Anliegen einzelner Patienten, einzelner einweisender Ärzte, einzelner Führungskräfte und Mitarbeiter berücksichtigt werden müssen, werden dort gefällt, wo ausreichend Informationen für die Bewertung und Gewichtung dieser Anliegen generiert werden können und wo auf diese Weise sichergestellt werden kann, dass diese Anliegen bei Entscheidungen angemessen berücksichtigt werden können. Das ist in der Regel in den unteren Leitungsebenen der Fall. Hinzu kommt, dass dort die Fähigkeit und die Bereitschaft, sich mit diesen Anliegen im Detail argumentativ auseinanderzusetzen und sie bei Entscheidungen zu berücksichtigen, stärker ausgeprägt sind als in anderen Leitungsebenen.

Das Zuweisen von Leitungsaufgaben ist Ausdruck des Vertrauens und der Wertschätzung, die die Geschäftsführung den Führungskräften und den Mitarbeitern entgegen bringt. Die Leitungsaufgaben müssen allerdings so gestaltet sein, dass auch das Bedürfnis der Verantwortungsträger nach Sicherheit, nämlich nach Sicherheit der sach- und zeitgerechten Bewältigung der übertragenen Leitungsaufgaben, befriedigt wird.

\section{Organisation der Leitungsorgane}

\section{a) Charakterisierung}

In dem vorangegangenen Kapitel wurde ausgeführt, welche Leitungsaufgaben welchem Leitungsgremium vernünftigerweise zugewiesen werden. Dabei wurde offen gelassen, wie die Binnenstruktur dieser Instanz beschaffen sein muss, damit diese ihrer Verantwortung im Sinne der Coldenen Regel nachkommen kann, und welche Regeln erforderlich sind, um das Wahrnehmen von Verantwortung sicherstellen zu können. Der Versuch der Beantwortung dieser noch offenen Frage ist Gegenstand der jetzt folgenden Betrachtung.

Die Binnenstruktur der Instanzen in der Spitze des Unternehmens ist durch die Art der in ihnen jeweils wahrzunehmenden Aufgaben und durch deren personelle Besetzung charakterisiert. Aber auch die Anzahl der Leitungsebenen und die Art, wie diese zusammenwirken, spielen bei der gesuchten Antwort auf die oben gestellte Frage eine Rolle ( $z u$ den verschiedenen Modellen der Krankenhausleitungsstruktur siehe Schmidt-Rettig, S. 226ff.); deshalb wird der Fokus auch auf diesen Aspekt der Organisation der Leitungsorgane gerichtet sein.

In der Praxis findet man mindestens vier Leitungsebenen vor, nämlich

- die Instanzen des Krankenhaus-Eigentümers(-Trägers) (1. Leitungsebene), 
- die Geschäftsführung (2. Leitungsebene),

- die Leitungen der Leistungsbereiche des Krankenhauses (3. Leitungsebene) und

- die diesen nachgeordneten Leistungsbereiche (wie z.B. Stationen - 4. Leitungsebene).

Bei größeren Krankenhaus-Verbünden gibt es zwischen der 1. und der 2. Leitungsebene nicht selten eine Zwischenebene (wie z.B. bei der SANA Kliniken AG). Diese ist meist regional ausgerichtet; sie wird im Zusammenhang mit den weiteren Betrachtungen der 1. Leitungsebene zugeordnet. Die folgenden Ausführungen beziehen sich auf die 2. Leitungsebene und auf die Schnittstellen zwischen den beiden obersten Leitungsebenen.

Der 2. Leitungsebene obliegt das Schaffen der Voraussetzungen für das Managen der Behandlungs-, Support- und Betriebsführungsprozesse in allen Leistungsbereichen des Krankenhauses. Sie ist für die Realisierung der im Regelfall von der 1. Leitungsebene festgelegten Unternehmensziele verantwortlich und kann zum Beispiel wie folgt organisiert sein:

- Das Krankenhaus wird durch einen Geschäftsführer als allein entscheidende Instanz geleitet; die diesem nachgeordnete Krankenhausleitung (im Regelfall mit dem Ärztlichen Direktor, dem Pflege- und dem Verwaltungsdirektor besetzt) ist ein Beratungsgremium ohne eigene Entscheidungs- und Weisungsbefugnis.

oder

- Das Krankenhaus wird durch einen Geschäftsführer geleitet. Die diesem nachgeordnete Krankenhausleitung ist ein Kollegialorgan, das mit eigenen Kompetenzen ausgestattet ist. Die Mitglieder des Kollegialorgans fällen entweder alle Entscheidungen gemeinsam, oder jedem Mitglied des Kollegialorgans ist ein eigener Kompetenzbereich mit eigener $\mathrm{Zu}$ ständigkeit übertragen worden; oder es gibt eine Kombination aus beidem. oder

- Es sind jeweils ein Geschäftsführer für a) die klinische Produktion und den Dienstleistungsbereich und b) den kaufmännischen Bereich vorgesehen - gegebenenfalls mit der Verpflichtung, bestimmte Entscheidungen gemeinsam zu fällen.

Wenn mehrere Personen mit der Leitung des Krankenhauses oder Teilen von diesem als Kollektiv beauftragt sind, bedeutet dies, dass die
Mitglieder des Kollektivs die Verantwortung für ihr Handeln gemeinsam tragen. Eine solche Regelung birgt die Gefahr in sich, dass die individuelle Verantwortung gemindert wird und sich das einzelne Mitglied des Cremiums zu Lasten des Gremiums oder einzelner seiner Mitglieder von Verantwortung entlasten kann (Küpper, S. 194). Das gilt für das Eintreten müssen für die Folgen sowohl des eigenen Handelns als auch für die der Mitarbeiter.

Die Leitungsarbeit in einem Krankenhaus ist geprägt durch die Zusammenarbeit unterschiedlicher Professionen. So sind die Instanzen, die Leitungsaufgaben wahrzunehmen haben, nicht selten durch mehrere Personen mit unterschiedlicher Qualifikation, also zum Beispiel mit einem Arzt, einer Krankenpflegekraft und einem Kaufmann besetzt. Dies gilt nicht nur für die Geschäftsführung, sondern gelegentlich auch für die Leitung der dieser nachgeordneten Organisationseinheiten ${ }^{56}$. Die Mitglieder der genannten Kollegien vertreten mitunter sehr unterschiedliche, berufsgruppenspezifische Interessen; ein fairer Interessenausgleich durch Bildung einer kollektiven Präferenzordnung aus den individuellen Präferenzordnungen ist ein außerordentlich schwieriger Weg (Küpper, S. 108ff. und die dort angegebene Literatur). Es gelingt deshalb im Regelfall auch nicht, ein gemeinsames Zielsystem zu entwickeln, das im Entscheidungsprozess für das Generieren von Lösungsalternativen und deren Bewertung benötigt wird.

Die Kompetenzverteilung zwischen der 1. und der 2. Leitungsebene ist oft unscharf. Die Ursachen dafür sind das Fehlen einschlägiger Kenntnisse bei den Mitgliedern der 1. Leitungsebene für die Notwendigkeit einer eindeutigen Kompetenzverteilung und die oft unzureichend entwickelte Sensibilität für die Folgen dieses Defizits. Zu den Ursachen des Defizits zählt schließlich die Art der Entwicklung der Kompetenzverteilung, nämlich ohne Beteiligung der Mitglieder der 2. Leitungsebene. Die Konsequenz dieser unklaren Zuständigkeiten ist eine - in Abhängigkeit von der Art der Persönlichkeit der Mitglieder der 2. Leitungsebene mehr oder weniger große - „gefühlte“ Ein-

56 Die Leitung des Klinikums der Charité - Universitätsmedizin Berlin zum Beispiel besteht aus dem Klinikumsdirektor, dem Ärztlichen Direktor und der Pflegedirektorin. Die Leitung der 17 CharitéCentren wird jeweils von einem Ärztlichen bzw. Wissenschaftlichen und einem Kaufmännischen Zentrumsleiter wahrgenommen. 
engung des Entscheidungsspielraums der 2. Leitungsebene.

\section{b) Anforderungen an die Gestaltung}

Aus den bisherigen Ausführungen lassen sich als Basis für das Ermöglichen sozial verantwortlichen Handelns vier Gestaltungs-Felder identifizieren:

1. die Verankerung von Verantwortung,

2. der Entscheidungsspielraum für die 2. Leitungsebene,

3. die Zusammenarbeit zwischen der 1. und der 2. Leitungsebene und

4. die Organisation der Interprofessionalität.

Aus der Sicht des Managements der sozialen Verantwortung stellen sich an die Organisation der Leitungsorgane folgende Anforderungen.

\section{Anforderungen an die Gestaltung der Organisation der Leitungsorgane}

\section{Gestaltungs-Feld, Anforderungen an die Gestaltung}

\section{Verankerung von Verantwortung}

Die zuständigen Gremien der 1. Leitungsebene sind gehalten, in Abstimmung mit der Geschäftsführung ein Regelsystem zu entwickeln und zu implementieren, mit dessen Hilfe die Wahrnehmung von Verantwortung - auch die Verantwortung durch Kollektive - im Unternehmensalltag verankert wird. Das Regelsystem zielt darauf ab, dass die Akteure des Krankenhauses, denen Verantwortung zugeschrieben wird, diese akzeptieren, internalisieren und praktizieren. Es präsentiert sich in mehreren (alternativen) Dimensionen (Küpper, S. 200ff.):

- Mit der Wahl der Rechtsform des Unternehmens wird die Verantwortung von Leitenden Mitarbeitern des Krankenhauses konkretisiert. Es werden die Rechtsfolgen für das nicht sach- und zeitgerechte Wahrnehmen der Verantwortung begründet.

- Grundsätze der Unternehmensführung legen fest, welcher Akteur welche Art von Verantwortung darüber hinaus trägt.

- In der Unternehmensverfassung werden die Konsequenzen festgelegt, die - zusätzlich zu den Rechtsfolgen - mit der Wahrnehmung oder der Verletzung von Verantwortung verbunden sind.

- In der Organisation des Krankenhauses wird der Grundsatz der Kongruenz von Entscheidungskompetenz und Verantwortung zum Ausdruck gebracht.

- Führungskräfte können über Führungsprinzipien und Führungsstil bei ihren Mitarbeitern das Bewusstsein und die Bereitschaft für die Übernahme von Verantwortung fördern.
- Für den Fall kollektiver Verantwortlichkeit empfiehlt es sich, Entscheidungen einstimmig fällen zu lassen (was nicht verhindern kann, dass sich das eine oder andere Mitglied des Kollektivs von der Verantwortung freizusprechen versucht), Abstimmungen tunlichst nicht geheim vornehmen zu lassen und Entscheidungsprozesse einschließlich des Abstimmungsverhaltens jedes Mitgliedes des Kollektivs möglichst detailliert zu dokumentieren.

- Vor allem empfiehlt es sich, einem Kollektiv der 1. und/ oder 2. Leitungsebene nur jene Leitungsaufgaben zuzuweisen, die essentiell sind für die Existenz und den Betrieb des Krankenhauses.

Die Wirksamkeit dieser Regeln kann dadurch sichergestellt werden, dass die Regeln

- unter Beachtung der Grundpostulate des Managements der sozialen Verantwortung entwickelt und implementiert,

- durch Anreize - positiver wie negativer Art - ergänzt und dass sie

- von Mitgliedern der 1. Leitungsebene und der Geschäftsführung konsequent vorgelebt werden.

Das Bedürfnis der Führungskräfte und der Mitarbeiter nach Wertschätzung und nach Sicherheit - die genannten Stakeholder sind sicher, dass sie dem zu erwartenden Maß an Verantwortung gerecht werden können - wird dadurch befriedigt. Die Fähigkeit sowohl der Mitglieder der Krankenhaus-Träger-Gremien, der Geschäftsführung als auch der Führungskräfte und der Mitarbeiter zur argumentativen, dialogischen Verständigung wird gestärkt und die Mündigkeit der genannten Stakeholder gefördert - beides unabdingbare Voraussetzungen für die Sensibilisierung der gesamten Belegschaft für die Ideen des Managements der sozialen Verantwortung und deren Realisierung.

\section{Entscheidungsspielraum für die 2. Leitungsebene}

Es entspricht der Grundidee des Managements der sozialen Verantwortung, wenn Entscheidungsspielräume geschaffen werden, die der Befriedigung der Bedürfnisse der Führungskräfte in der 2. Leitungsebene nach persönlicher Entfaltung, nach Selbstständigkeit und nach Wertschätzung dienen. Die Kompetenzzuordnung sollte so erfolgen, dass daraus ein möglichst hohes Maß an Vertrauen, das den Mitgliedern der 2. Leitungsebene durch den Eigentümer/Träger geschenkt wird, deutlich wird.

Um in diesem Sinne dauerhaft wirksam werden zu können, ist der Entscheidungsspielraum das Ergebnis einer argumentativen, dialogischen Verständigung zwischen den Vertretern der 1. und 2. Leitungsebene. Als Ergebnis dieses Dialogs sind die Kompetenzen für die beiden Leitungsebenen in der Satzung des Krankenhauses und in Stellenbeschreibung(en) für die Geschäftsführung so beschrieben, dass Fehldeutungen möglichst vermieden werden können. 
Die Festlegung einer eindeutigen Verteilung der Leitungskompetenzen auf die beiden obersten Leitungsebenen ist die eine Seite der Medaille. Die gewünschte Wirkung wird allerdings nur dann eintreten, wenn die vereinbarte Verteilung auch respektiert wird. Es ist deshalb zu fordern, dass die für das Krankenhaus zuständige Instanz des Krankenhaus-Eigentümers(-Trägers) nur die Aufgaben wahrnimmt, die ihr durch die Satzung oder ähnliche Dokumente zugeschrieben worden sind; sie versucht auch nicht, informell auf Entscheidungen der 2. Leitungsebene Einfluss zu nehmen. Die Vertreter der 2. Leitungsebene werden nicht versuchen, sich durch „Rückdelegation“ von Kompetenzen von Verantwortung zu entlasten.

\section{Zusammenarbeit zwischen der 1. und}

\section{der 2. Leitungsebene}

Verantwortliches Handeln der Geschäftsführung in der 2. Leitungsebene wird gefördert, wenn dieses möglichst frühzeitig im Sinne einer argumentativen, dialogischen Verständigung in die durch systematische StakeholderAnalysen vorbereiteten unternehmerischen Entscheidungen der 1. Leitungsebene einbezogen wird. Die Vertreter der 2. Leitungsebene erhalten damit die Möglichkeit, als Agenten vieler von diesen Entscheidungen Betroffener deren Anliegen geltend zu machen.

Dieses setzt ein hohes Maß an Sachkunde methodischer Art bei den Entscheidungsträgern in der 1. Leitungsebene voraus. Sie müssen in der Lage und bereit sein, diesen Dialog zu führen. Der Krankenhaus-Eigentümer wird deshalb aufgefordert, in die Professionalisierung der Entscheidungsträger in der 1. Leitungsebene zu investieren.

\section{Organisation der Interprofessionalität}

Es ist zu fordern, dass Führungskonzepte entwickelt und implementiert werden, die dem Abbau der Spannungen zwischen den Berufsgruppen Rechnung tragen. Einzelheiten dazu siehe Kapitel 4.2.8, Notwendigkeit, Interprofessionalität zu organisieren und Kapitel 6.4.2, Personalarbeit.

\subsection{Die betriebliche Leitungsentscheidung als wertende Parteinahme}

\subsubsection{Einführung}

Für das Ermöglichen und Realisieren sozial verantwortlichen Handelns kommt es nicht nur darauf an, wie die verschiedenen Komponenten der Leitungsstruktur eines Unternehmens gestaltet sind. Maßgeblichen Einfluss darauf, ob überhaupt und in welchem Maße die betriebliche Leitungstätigkeit den - möglicherweise antinomischen oder konkurrierenden - Anliegen der Stake- holder Rechnung trägt, hängt auch davon ab, wie der durch die organisatorische Grundstruktur des Krankenhauses gesetzte Rahmen genutzt wird, und somit von der Art und Weise, wie der Prozess der betrieblichen Leitungsentscheidung organisiert ist - das heißt vor allem, wer mit welchem Beitrag an dem Zustandekommen einer Entscheidung mit welcher Verbindlichkeit beteiligt ist.

Um den Zusammenhang zwischen Entscheidung und verantwortlichem Handeln analysieren und daraus Gestaltungsempfehlungen ableiten zu können, bedarf es zunächst einer Verständigung darüber, was unter einer betrieblichen Leitungsentscheidung verstanden werden soll und in welchen Ebenen und Phasen der Entscheidungsprozess realisiert wird (s. Kap. 6.5.2). Die Gestaltung des Entscheidungsprozesses im Sinne des Managements der sozialen Verantwortung (s. Kap. 6.5.4) ist abhängig von der Art der jeweils zu fällenden betrieblichen Leitungsentscheidung, die deshalb zunächst betrachtet werden muss (s. Kap. 6.5.3).

\subsubsection{Begriff, Phasen und Ebenen betrieblicher Leitungsentscheidungen}

\section{Begriff}

Eine Entscheidung wird immer dann notwendig, wenn ein für einen Aufgabenbereich Verantwortlicher oder ein Dritter mit dem derzeitigen und/ oder mit dem zukünftig zu erwartenden betrieblichen Geschehen nicht zufrieden und der Meinung ist, dass sich mit Hilfe bestimmter Handlungen, die als Ergebnis der zu fällenden Entscheidung realisiert werden, eine Verbesserung der Situation erreichen lässt. Die Mangelempfindung bewirkt, dass der für den betroffenen Aufgabenbereich Verantwortliche den Prozess der Problemlösung initiiert oder dass der Dritte den Verantwortlichen zu der Lösung des Problems veranlasst.

Der Entscheidungsträger muss zu einem Entschluss kommen, obwohl sein Wissen möglicherweise unvollständig ist und die ihm zur Verfügung stehenden Daten unsicher sind; die Konsequenzen seines Handelns kann er nicht eindeutig und nicht mit Sicherheit voraussehen. Es gilt deshalb, mit den dafür zur Verfügung stehenden Instrumenten die Art und den Grad an Unsicherheit und die Wirkungen von Chancen und Risiken zu untersuchen; die Ergebnisse werden mit Zielvor- 
stellungen unterschiedlicher Risikobereitschaft verknüpft (Küpper, S. 193).

Das Risiko unerwünschter Handlungsfolgen trägt der Entscheidende in vielen Fällen nicht allein - indem ihm zum Beispiel die vereinbarte Prämie wegen Nichterfüllens der vereinbarten Ziele nicht ausgezahlt wird -, sondern vielfach auch der von der Entscheidung Betroffene. Letzteres gilt vor allem für den Patienten, dessen Gesundheit in Folge einer Fehlentscheidung des behandelnden Arztes nicht wieder hergestellt werden konnte, oder für den Eigentümer des Krankenhauses, der wegen Managementfehler seine Renditeerwartungen nach unten korrigieren muss.

Betriebliche Leitungsentscheidungen zeichnen sich durch ihre auf das Unternehmen und dessen relevante Umwelt begrenzte Wirkung aus sowie durch die Weisungsbefugnis, mit der der Entscheidungsträger ausgestattet ist. Betriebliche Leitungsentscheidungen werden in allen Leitungsebenen des Krankenhauses gefällt. Sie differieren hinsichtlich der unterschiedlichen Reichweite und des unterschiedlichen Gewichts ihrer Wirkung. Sie heben sich voneinander durch den Entscheidungsspielraum ab, der den Entscheidungsträgern in den einzelnen Leitungsebenen in ungleichem Maß zur Verfügung steht.

Vor allem als Folge zunehmender Spezialisierungen nimmt der Anteil betrieblicher Leitungsentscheidungen $\mathrm{zu}$, bei denen die Verantwortung für das Ergebnis einer Entscheidung einer Person und die Verantwortung für das Umsetzen des Entscheidungsergebnisses einer zweiten Person zugeschrieben sind. Beide Personen können ihre Verantwortung für das Nicht-Erreichen des gesteckten Zieles auf die jeweils andere Person abschieben mit dem Hinweis, sie habe nur eine Anordnung ausgeführt (die ausführende Person) bzw. sie habe sich auf die richtige Durchführung der Anordnung verlassen müssen (die entscheidende Person). Die Gefahr des Nicht-Erreichens des Zieles ist systemimmanent erzeugt und gleichzeitig systemimmanent nicht zurechenbar (Beck, S. 104).

\section{Beispiel}

Der einen Patienten behandelnde Arzt legt fest, welche Maßnahmen der Behandlungspflege durchgeführt werden sollen; er ordnet diese Maßnahmen einer Pflegekraft zur Durchführung an. Die Pflegekraft setzt das Ergebnis der Entscheidung um.
Der Arzt ist verantwortlich für die Festlegung der richtigen Therapiemaßnahmen und dafür, dass er die Durchführung der Maßnahmen einer dafür ausreichend qualifizierten Pflegekraft angeordnet hat. Die Pflegekraft ist für die sach- und zeitgemäße Realisierung der Maßnahmen verantwortlich.

Wenn das Behandlungsziel nicht erreicht wird, werden der Arzt und die Pflegekraft die Ursachen dafür gegebenenfalls bei dem jeweils anderen Mitglied des Behandlungsteams vermuten und die Verantwortung für den Misserfolg ablehnen.

\section{Ebenen}

Das Fällen betrieblicher Leitungsentscheidungen ist ein außerordentlich komplexer Prozess, dessen Teil-Prozesse sich - zumindest gedanklich drei Ebenen zuordnen lassen (s. Abb. 43). Diese Ebenen werden an dieser Stelle zunächst nur skizziert; eine detaillierte Beschreibung erfolgt nach diesem Überblick im Zusammenhang mit der Präsentation der Entscheidungs-Phasen im nächsten Kapitel.

1. In der Ebene 1 wird entschieden, welche Teilfunktion betrieblicher Leitungstätigkeit - z.B. die Planung des Personalbedarfs - realisiert werden soll.

2. Diese Festlegung löst in der Ebene 2 einen Entscheidungsprozess aus, mit dessen Hilfe das Ergebnis der Teilfunktion betrieblicher Leitungstätigkeit - z.B. der Personalbedarf - ermittelt wird.

3. Der Entscheidungsprozess besteht aus mehreren Teil-Prozessen, die in der Ebene 3 jeweils spezifische Vor-Entscheidungen fordern -z.B. darüber, welche Stakeholder mit welchem Gewicht bei der Ermittlung des Personalbedarfs - berücksichtigt werden sollen.

\section{Phasen}

Die Entscheidung besteht in der Festlegung des einzuschlagenden Lösungsweges (= Entscheidung im engeren Sinne) sowie in deren Vorbereitung. Mit der Entscheidung wird ein bestimmter zukünftiger Zustand vor einem anderen Zustand bevorzugt.

Vier Phasen des Entscheidungsprozesses lassen sich unterscheiden (s. Abb. 43, Ebene 2):

1. Feststellung und genaue Beschreibung des zu lösenden Problems, 


\subsection{Die betriebliche Leitungsentscheidung als wertende Parteinahme}
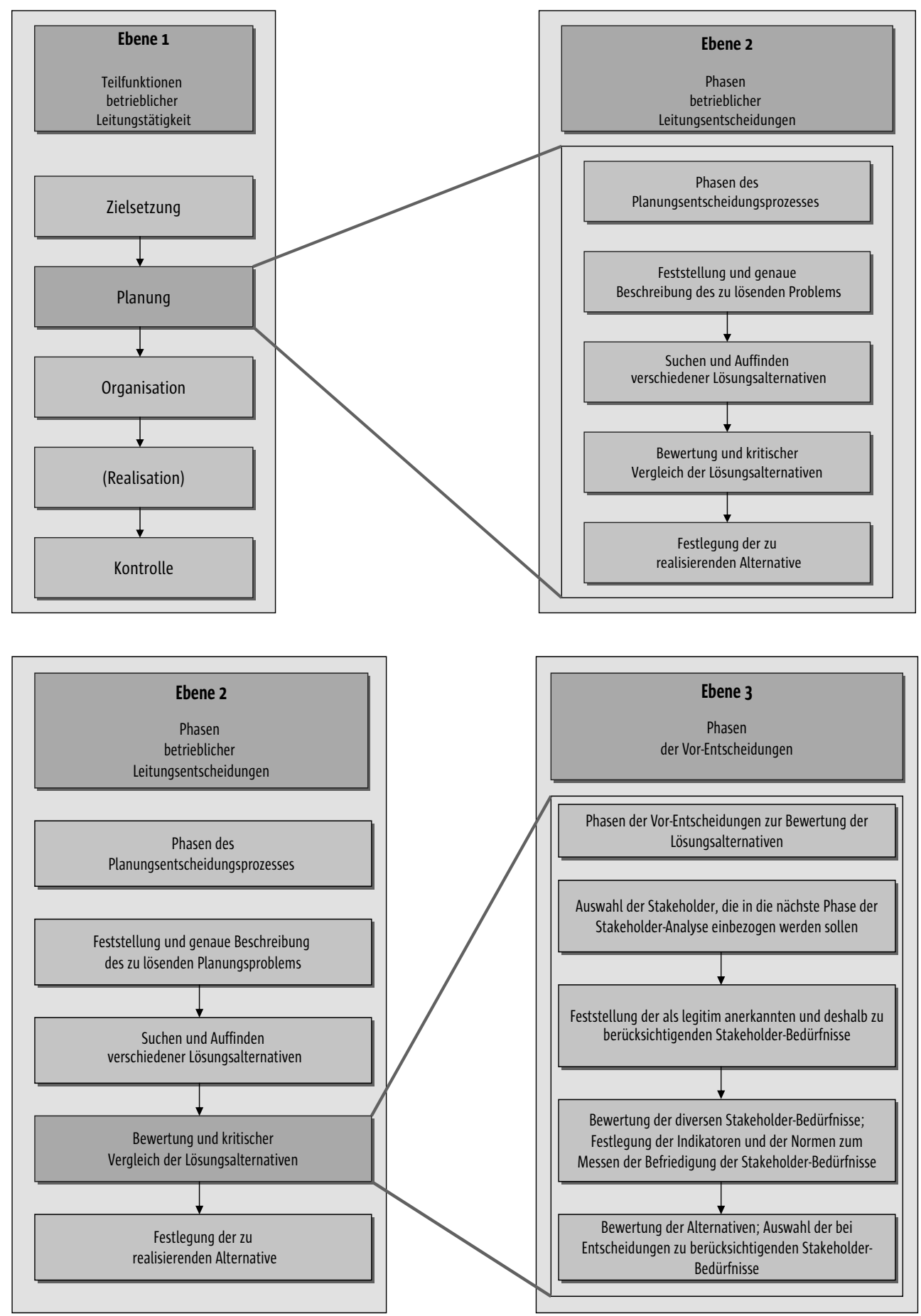

Abb. 43 Ebenen 1, 2 und 3 betrieblicher Leitungsentscheidungen (in Anlehnung an Naegler et al., S. 250) 
2. Suchen und Auffinden verschiedener Lösungsalternativen,

3. Bewertung und kritischer Vergleich der Lösungsalternativen und

4. Festlegung der zu realisierenden Alternative.

Der Phase 4 des Entscheidungsprozesses schließt sich das Umsetzen des Ergebnisses der Entscheidung und dessen Evaluierung an.

Kennzeichnendes Merkmal der Entscheidung ist also die Existenz mehrerer Alternativen, zwischen denen eine Wahl getroffen wird. Dabei werden der Verzicht auf die Einbeziehung einer bestimmten Alternative in die Phasen 3 und 4 oder auf eine Lösung des Problems überhaupt schon als Alternative in dem oben genannten Sinne angesehen. Beide Arten von Verzicht erfordern eine wertende Begründung.

Der Entscheidungsprozess wurde bisher in idealtypischer Betrachtungsweise als ein linearer Vorgang dargestellt, an dessen Ende die Entscheidung i.e.S. gefällt wird, nämlich die Auswahl und die Festlegung des einzuschlagenden Lösungsweges. Tatsächlich ist die Entscheidung ein höchst komplexer Prozess, der von Rückkopplungen und Einzelproblemlösungen überlagert wird. In jeder Phase des Entscheidungsprozesses werden Vor-Entscheidungen gefällt und damit der Input für die nächste Phase des Entscheidungsprozesses festgelegt (s. das Beispiel „Bewertung und kritischer Vergleich der Lösungsalternativen“ in Abb. 43, Ebene 2 und 3).

Besonders mit Blick auf das Management der sozialen Verantwortung und die in diesem $\mathrm{Zu}$ sammenhang zu fällenden Entscheidungen ist diese differenzierte Analyse des Entscheidungsprozesses von Bedeutung. Dabei spielt vor allem die Phase 3 eine zentrale Rolle (s. Abb. 43, Ebene 2 und 3). Die Bewertung und der kritische Vergleich der Lösungsalternativen dienen dann nämlich nicht nur der Feststellung, welchen Beitrag die verschiedenen Lösungsalternativen zum Erreichen der Unternehmensziele leisten. Es gilt auch herauszufinden, welchen Beitrag die verschiedenen Alternativen zur Befriedigung der Stakeholder-Bedürfnisse leisten. Bei allen betrieblichen Leitungsentscheidungen werden bei der Bewertung der Lösungsalternativen die daraus sich ergebenden Folgen für die Stakeholder daraufhin überprüft, ob diese gerechtfertigt werden können (Küpper, S. 136).

Im Zusammenhang mit der Realisierung der Phase 3 einer Planungsentscheidung und zwecks
Berücksichtigung der diversen Stakeholder-Anliegen sind vier für den Planungsprozess spezifische und hier beispielhaft dargestellte Vor-Entscheidungen zu fällen (s. Abb. 43, Ebene 3):

1. Identifizierung der Stakeholder und deren Gewichtung; Auswahl der Stakeholder, die in die nächste Phase der Stakeholder-Analyse einbezogen werden sollen,

2. Feststellung der als legitim anerkannten und deshalb zu berücksichtigenden StakeholderBedürfnisse,

3. Bewertung der diversen Stakeholder-Bedürfnisse; Festlegung der Indikatoren und der Normen zum Messen der Befriedigung der Stakeholder-Bedürfnisse und

4. Bewertung der Alternativen; Auswahl der bei Entscheidungen zu berücksichtigenden Stakeholder-Bedürfnisse.

Möglicherweise muss ein Entscheidungsprozess wiederholt werden, weil der Beitrag des einzuschlagenden Lösungsweges zur Realisierung des Unternehmenszieles, der unter Berücksichtigung der Stakeholder-Anliegen ermittelt worden ist, nicht akzeptiert werden kann. Dann müssen die Gewichte als Basis für die Berücksichtigung der Stakeholder überprüft und gegebenenfalls geändert werden mit dem Ergebnis, dass möglicherweise andere Stakeholder in den Entscheidungsprozess einbezogen werden. Auch die Bewertung der Stakeholder-Bedürfnisse muss hinterfragt und eventuell geändert, damit am Ende eine für das Krankenhaus und die Stakeholder akzeptable Lösung gefunden werden kann.

Die besondere Schwierigkeit der Alternativenbewertung und damit der Entscheidungsfindung ergibt sich aus dem Umstand, dass

1. es in der Praxis sehr häufig gilt, mehrere Ziele bzw. Bedürfnisse zu berücksichtigen, die nicht selten antinomisch sind oder in Konkurrenz zueinander stehen, und

2. die Entscheidungen in Kollektiven gefällt werden, deren Mitglieder unterschiedliche Präferenzordnungen haben und deshalb die Alternativen unterschiedlich bewerten.

Für die Lösung dieses Problems stehen mehrere Ansätze zur Verfügung, die allerdings nicht Gegenstand dieser Untersuchung sein können. Es wird auf die umfangreiche Spezialliteratur verwiesen. 


\subsubsection{Arten betrieblicher Leitungsentscheidungen}

Die betrieblichen Leitungsentscheidungen lassen sich hinsichtlich

1. ihres Zwecks in Zielsetzungsentscheidungen und Zielerreichungs-(= Mittel-)entscheidungen

2. ihres Anlasses in konstitutive Entscheidungen und situative Entscheidungen und hinsichtlich

3. der Entscheidungssituation in innovative Entscheidungen und in Routine-Entscheidungen unterscheiden.

Jede betriebliche Leitungsentscheidung lässt sich anhand dieser Merkmale charakterisieren. Man kann insofern acht Arten von Entscheidungen unterscheiden. Zum besseren Verständnis der weiteren Ausführungen ist es allerdings ausreichend, nur die Charakteristika der innovativen und der Routine-Entscheidungen kurz vorzustellen: Routineentscheidungen zeichnen sich dadurch aus, dass das zu lösende Problem wohldefiniert ist; es gibt fertige Programme, mit deren Hilfe die Entscheidung vorbereitet und gefällt werden kann. Das Vorbereiten und Fällen innovativer Entscheidungen setzt dagegen voraus, dass vor dem Start des eigentlichen Entscheidungsprozesses mittels aufwändiger Verfahren sowohl die Problembeschreibung als auch das Lösungsprogramm erarbeitet werden. Der Alltag des Krankenhaus-Managements kennt überwiegend die Routine-Entscheidung; deshalb ist nur diese der Gegenstand der weiteren Untersuchung.

Von den bisher skizzierten „echten“ Entscheidungen werden habituelles Verhalten und impulsives Verhalten unterschieden. Habituelles Verhalten zeichnet sich dadurch aus, dass der Entscheider auf seine Erfahrungen zurück greift und das in die Praxis umsetzt, was er in ähnlichen Situationen in der Vergangenheit schon immer gemacht hat. Gegebenenfalls wird die Beschreibung des Problems so angepasst, dass eine schon erprobte Lösung angewandt werden kann. Bei impulsivem Verhalten verlässt sich der Entscheidungsträger auf ihm zufällig zugegangene Informationen, um aus ihnen den einzuschlagenden Lösungsweg zu entwickeln.

Bei beiden Arten von „unechter“ Entscheidung findet eine systematische Vorbereitung der Entscheidung in den Ebenen 2 und 3 betrieblicher Leitungsentscheidungen (s. Abb. 43) und eine Entscheidung i.e.S., nämlich die Auswahl unter mehreren bewerteten Alternativen, nicht statt. Auch gilt nur für die „echten“ Entscheidungen, dass der Entscheidungsprozess einer willentlichen Gestaltung zugänglich ist. Nur im Rahmen eines derart organisierten Entscheidungsprozesses können Stakeholder-Bedürfnisse systematisch in Entscheidungen einbezogen werden - ist also sozial verantwortliches Handeln möglich. Nur diese Art von Entscheidungsprozess ist deshalb Gegenstand der weiteren Betrachtung.

\subsubsection{Organisation des Entscheidungsprozesses}

\section{Charakterisierung}

Ob das Ergebnis einer unternehmerischen Entscheidung in unternehmerisches Handeln mündet, dessen Folgen im Sinne der Grundidee des Managements der sozialen Verantwortung qualifiziert werden kann, hängt entscheidend davon $\mathrm{ab}$, wie der Prozess des Entscheidens gehandhabt wird. In diesem Zusammenhang stellen sich vor allem drei Fragen:

1. Einerseits hängt das Ergebnis der Entscheidung davon ab, ob die Entscheidung von einer einzelnen Person oder von einem Kollektiv gefällt wird.

2. Wenn mehrere Personen an dem Entscheidungsprozess mitwirken, kommt es darauf an, an welcher Phase der betrieblichen Leitungsentscheidung und an welchen gegebenenfalls notwendigen Vor-Entscheidungen die verschiedenen Personen mit welcher Verbindlichkeit beteiligt werden.

3. Schließlich wird das Ergebnis der Entscheidung dadurch bestimmt, ob die Organisation des Entscheidungsprozesses in Führungsgrundsätzen für alle Entscheidungsträger verbindlich vorgegeben ist oder ob Entscheidungsträger fallweise festlegen dürfen, wen sie an einer Entscheidung auf welche Art und mit welcher Verbindlichkeit beteiligen.

In dem vorliegenden Kapitel wird die Organisation der Routineentscheidung zum Thema gemacht. Gegenstand der Betrachtung ist die Ebene 2 betrieblicher Leitungsentscheidungen.

Wenn an einer Entscheidung - wie für die weitere Betrachtung vorausgesetzt wird - mehrere Personen beteiligt sind, gibt es für deren Zusammenwirken mehrere Möglichkeiten (s. Abb. 44): 1. Es sind mehrere Personen als Kollektiv gemeinsam und gleichberechtigt an einem Ent- 


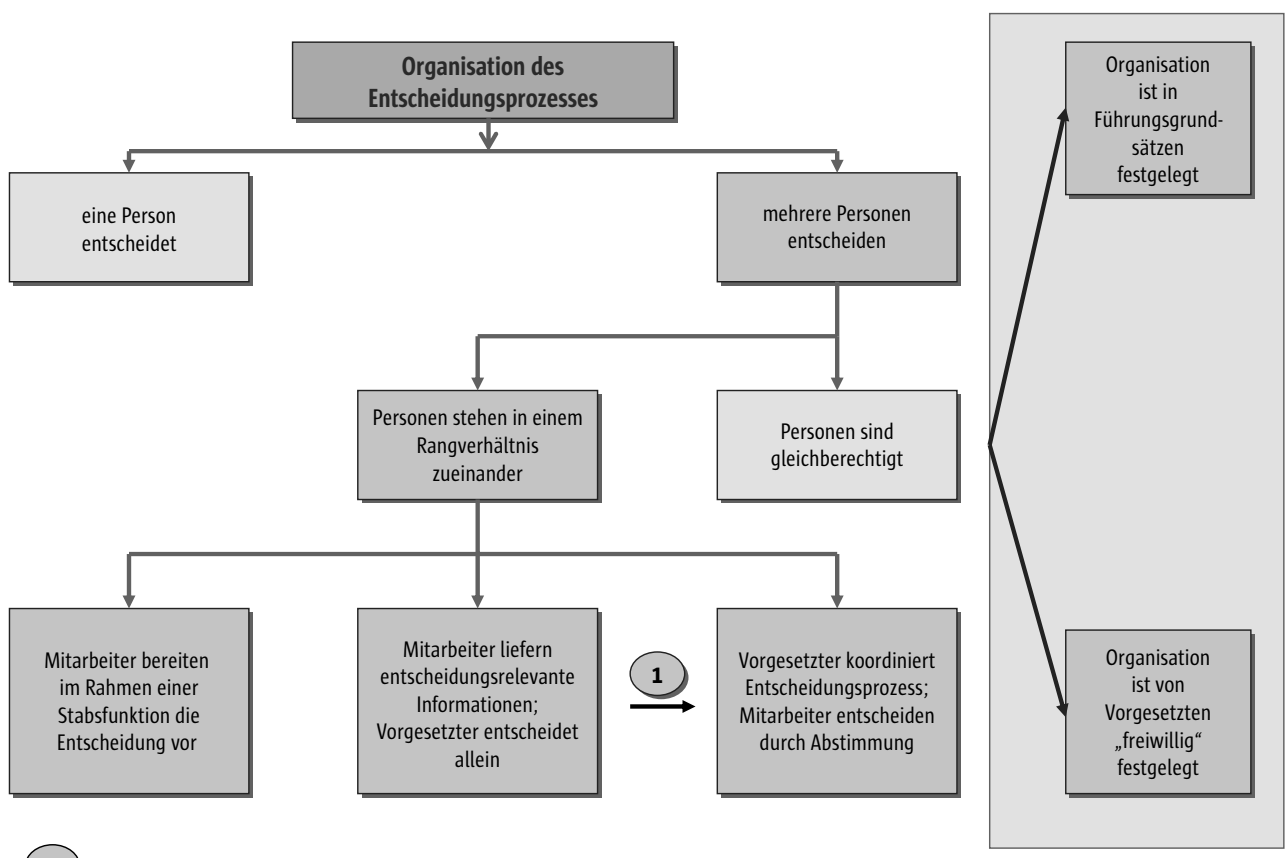

1 Führungsstil-Kontinuum (Tannenbaum; Schmidt, S. 96)

Abb. 44 Organisation des Entscheidungsprozesses

scheidungsprozess beteiligt (als Beispiel wurde das Kollegialorgan „Krankenhausleitung“ schon genannt). Die Mitglieder des Kollektivs tragen gemeinsam die mit der Leitungstätigkeit verbundene Verantwortung.

Damit stellt sich ein Gestaltungs-Problem, das eher dem Bereich „Organisation der Leitungsorgane" zugeordnet werden kann: es wird deshalb hier nicht behandelt.

2. Die Leitung einer Organisationseinheit ist einer Person übertragen worden, der mehrere Mitarbeiter zuarbeiten. Die Führungskraft beteiligt ihre Mitarbeiter an den zu fällenden Entscheidungen. Für das Zusammenwirken der Führungskraft mit ihren Mitarbeitern gibt es mehrere Optionen:

a) Die Führungskraft beteiligt ihre Mitarbeiter als Stabsstelle an den Entscheidungsphasen 2 und 3 zwecks Vorbereitung einer Entscheidung. Die Feststellung und genaue Beschreibung des zu lösenden Problems (Phase 1) sowie die Auswahl des einzuschlagenden Lösungsweges (Phase 4) behält sich die Führungskraft vor. Die Führungskraft beschreibt das Problem und beauftragt die
Mitarbeiter mit der Vorbereitung der Entscheidung (= Anordnungsverantwortung). Die Mitarbeiter legen einen begründeten Vorschlag für die Festlegung des einzuschlagenden Lösungsweges vor (= Übernahme- und Durchführungsverantwortung).

b) Die Führungskraft beteiligt ihre Mitarbeiter an allen Phasen des Entscheidungsprozesses. Auch die Festlegung des einzuschlagenden Lösungsweges erfolgt gemeinsam. Die Art und Weise, wie die Cruppe in den einzelnen Phasen des Entscheidungsprozesses zu den Ergebnissen kommt, die in der jeweils folgenden Phase als Input benötigt wird, sowie die Verteilung der Verantwortung auf die Führungskraft und ihre Mitarbeiter hängen von dem in einem Krankenhaus gepflegten Führungsstil ab: Von der Lieferung der für die Entscheidung notwendigen Informationen durch die Mitarbeiter an den danach alleine entscheidenden Vorgesetzten, der die Cründe seiner Entscheidung seinen Mitarbeitern nicht bekannt gibt, bis hin zu der Koordination des Entscheidungsprozesses durch 
den Vorgesetzten und einer Mehrheitsentscheidung durch die Mitarbeiter-Cruppe einschließlich der Führungskraft reichen die Grundformen der Führungsstile (Tannenbaum; Schmidt, S. 96).

Die Art der Beteiligung der Mitarbeiter an Entscheidungsprozessen ist an verschiedene Voraussetzungen gebunden. Die Eigenschaften des Vorgesetzten und die seiner Mitarbeiter sowie die Art des zu lösenden Problems und die Bedingungen für die Lösung des Problems (Bühner, S. 198) sind dafür maßgeblich, welcher Führungsstil zum Einsatz kommt und wie der Entscheidungsprozess organisiert wird.

In der Praxis der betrieblichen Leitungstätigkeit kann nicht selten beobachtet werden, dass es - soweit rechtliche Normen dem nicht entgegenstehen ${ }^{57}$ - in das Belieben des Vorgesetzten gestellt ist, an welchen Entscheidungen er seine Mitarbeiter in welcher Form beteiligt. In anderen Krankenhäusern gibt es neben den rechtlichen Normen Führungsgrundsätze, in denen die Art der Beteiligung der Mitarbeiter an Entscheidungsprozessen fixiert ist. Wenn Letzteres der Fall ist, wissen die Mitarbeiter, an welchen Entscheidungen sie auf welche Weise beteiligt werden und können die Beteiligung gegebenenfalls einfordern.

\section{Anforderungen an die Gestaltung}

Mit der Gestaltung des Entscheidungsprozesses kann das Wirksamwerden des Managements der sozialen Verantwortung auf zwei Ebenen gefördert werden:

- Zunächst geht es um die Beantwortung der Frage, wie der Entscheidungsprozess gestaltet sein muss, um organisationale Barrieren, die das Wahrnehmen von Verantwortung behindern könnten, nicht entstehen zu lassen bzw. abzubauen.

- Mit der Modellierung des Entscheidungsprozesses im Sinne der Goldenen Regel kommt

57 So ist in den verschiedenen Personalvertretungsgesetzen (s. zum Beispiel $\oint 75$ Bundespersonalvertretungsgesetz) vorgeschrieben, bei welchen Entscheidungen die Personalvertretung beteiligt (= um Zustimmung gefragt) werden muss oder mitwirken kann (= angehört werden muss). die Geschäftsführung ihrer Verantwortung gegenüber ihren Mitarbeitern nach. Sie leistet einen Beitrag zur Befriedigung einer ganzen Reihe von Mitarbeiter-Bedürfnissen und motiviert damit die Mitarbeiter, sozial handeln zu wollen.

Ausgehend von diesen Gestaltungszielen und den verschiedenen Charakteristika des Entscheidungsprozesses lassen sich fünf Cestaltungs-Felder identifizieren (s. folgenden Abschnitt):

1. Entscheidungsspielraum des Verantwortungsträgers,

2. Beteiligung der Mitarbeiter als Stabsstelle an Entscheidungsprozessen,

3. Beteiligung der Mitarbeiter an allen Phasen des Entscheidungsprozesses,

4. Beteiligung anderer Stakeholder an Entscheidungsprozessen und

5. Behandlung des mit einer Entscheidung verbundenen Risikos (Küpper, S. 193).

\section{Anforderungen an die Organisation des Entscheidungsprozesses}

\section{Gestaltungs-Feld, Anforderungen an die Gestaltung}

\section{Entscheidungsspielraum des Verantwortungsträgers}

Der Verantwortungsträger kann sozial verantwortlich handeln und die Verantwortung für die Folgen einer Entscheidung nur dann übernehmen, wenn die in Kapitel. 5.3.2 beschriebenen Voraussetzungen erfüllt sind: Der Entscheidungsspielraum ist eindeutig festgelegt und mit dem Verantwortungsspielraum deckungsgleich; die Chancen für die Realisierung verantwortlichen Handelns liegen innerhalb des Entscheidungsspielraums.

Der Entscheidungsspielraum ist ein Indikator für die Realisierung sozialer Verantwortung im Sinne von Mitarbeiterorientierung:

- Der Entscheidungsspielraum ist das Ergebnis einer argumentativen, dialogischen Verständigung.

- In der Gestaltung des Entscheidungsspielraums kommt zum Ausdruck, inwieweit das Bedürfnis der Verantwortungsträger, denen die Entscheidungs-Befugnis übertragen worden ist, nach Selbstständigkeit und Selbstentfaltung befriedigt werden soll. In dem Ausmaß des Entscheidungsspielraums wird schließlich der Grad an Wertschätzung und Vertrauen manifest, die dem Verantwortungsträger entgegengebracht wird.

- Das Bedürfnis der Verantwortungsträger nach Sicherheit wird befriedigt, wenn in Führungsgrundsätzen, in dem Arbeitsvertrag oder einem anderen Dokument der Entscheidungsspielraum eindeutig beschrieben wird. 
Damit sich die Übertragung von Entscheidungsbefugnissen in verantwortlichem Handeln niederschlägt, ist von dem Entscheidungsträger zu fordern, dass er den ihm eingeräumten Spielraum verantwortungsvoll nutzt. Die Geschäftsführung unterstützt den Entscheidungsträger dabei durch die Förderung der Fähigkeit zur argumentativen, dialogischen Verständigung und durch die Entwicklung der Entscheidungsträger zu mündigen Mitarbeitern. Sie akzeptiert das Realisieren „unechter“ Entscheidungen nur dann, wenn das Vorliegen der Voraussetzungen dafür nachgewiesen wird.

\section{Beteiligung der Mitarbeiter als Stabsstelle an Entscheidungsprozessen}

Hinsichtlich der Zuweisung der Übernahme- und Durchführungsverantwortung an die Mitarbeiter gilt das unter dem ersten Gestaltungs-Feld gesagte analog.

Für die Mitarbeiter ist es ein Zeichen von Wertschätzung und des in sie gesetzte Vertrauens, wenn ihnen die Vorbereitung von Entscheidungen übertragen wird. Im Sinne des Managements der sozialen Verantwortung ist es, wenn die Führungskraft - ausgehend von einer Empfehlung ihrer Mitarbeiter - die Auswahl des einzuschlagenden Lösungsweges auf der Grundlage einer argumentativen, dialogischen Verständigung mit ihren Mitarbeitern realisiert. Wenn die Führungskraft entgegen der Empfehlung ihrer Mitarbeiter entscheidet, dann begründet sie diese Entscheidung.

Es kommt dem Bedürfnis der Mitarbeiter nach Sicherheit entgegen, wenn in einem Führungskonzept, in dem Arbeitsvertrag oder in einem anderen Dokument die Rolle beschrieben wird, die ihnen im Rahmen des Entscheidungsprozesses zugeschrieben wird. Es sollte nicht im Belieben des Verantwortungsträgers liegen, den Mitarbeitern zum Beispiel nur das Sammeln von Information für die Realisierung der verschiedenen Phasen des Entscheidungsprozesses zu übertragen, während die anspruchsvolleren Arbeiten - wie z.B. die Erarbeitung eines begründeten Entscheidungs-Vorschlages - von ihm selbst durchgeführt werden.

\section{Beteiligung der Mitarbeiter an allen Phasen \\ des Entscheidungsprozesses}

Hinsichtlich der Zuweisung der Verantwortung für die Folgen bestimmter Entscheidungen an die Führungskraft und deren Mitarbeiter gilt das unter dem ersten GestaltungsFeld gesagte analog.

Für die Mitarbeiter ist es ein Zeichen von Wertschätzung und des in sie gesetzte Vertrauens, wenn sie an dem Fällen von Entscheidungen nicht nur als Stabs-Mitarbeiter, sondern möglichst im Sinne der partizipativen Führung und auf der Grundlage einer argumentativen, dialogischen Verständigung beteiligt werden. Für den Fall, dass Vorge- setzte nach einer Beratung mit ihren Mitarbeitern alleine und gegebenenfalls gegen deren Empfehlung entscheiden, ist von den Vorgesetzten zu fordern, dass sie das Ergebnis ihrer Entscheidung begründen.

Es kommt dem Bedürfnis der Mitarbeiter nach Sicherheit entgegen, wenn in einem Führungskonzept, in dem Arbeitsvertrag oder in einem anderen Dokument festgelegt ist, in welcher Entscheidungssituation sie mit welcher Art von Beteiligung rechnen können.

Wenn die Geschäftsführung die Art der Beteiligung der Mitarbeiter für jeweils bestimmte Entscheidungssituationen in einem Führungskonzept fixieren will, muss sie prüfen, ob die für den in Abhängigkeit von der Entscheidungssituation präferierten Führungsstil notwendigen personellen Voraussetzungen gegeben sind (Bühner, S. 198). Es ist zu fordern - damit ein weitgehend partizipativer Führungsstil praktiziert werden kann -, dass die Führungskraft und/oder die Mitarbeiter gegebenenfalls zu einer einschlägigen Weiterbildung verpflichtet werden.

\section{Beteiligung anderer Stakeholder \\ an Entscheidungsprozessen}

Es ist zu fordern zuzulassen, dass die Stakeholder ihre Interessen im Entscheidungsprozess möglichst im Detail vertreten können und dass die dafür erforderlichen personellen Voraussetzungen geschaffen werden. Die Verantwortungsträger verpflichten sich,

- die Folgen ihrer Entscheidungen explizit daraufhin zu überprüfen, ob sie im Sinne der Goldenen Regel gerechtfertigt werden können,

- den Stakeholdern zu ermöglichen, ihre Interessen im Entscheidungsprozess persönlich oder durch Vertreter wahrzunehmen und

- ihre Entscheidungen gegenüber den Stakeholdern zu begründen.

\section{Behandlung des mit einer Entscheidung verbundenen Risikos}

Entscheidungen werden nicht selten angesichts unvollkommener Informationen und unsicherer Erwartungen über künftige Entwicklungen gefällt. Sie sind mit dem Risiko behaftet, dass das Ziel, das verfolgt wird, nicht oder nur mit einem geringeren Erfüllungsgrad erreicht wird. Es stellt sich deshalb die Frage, wer das Risiko des Verfehlens der gesteckten Ziele in welchem Ausmaß trägt. Die Beantwortung dieser Frage ist umso schwieriger dann, wenn die Entscheidung im Kollektiv gefällt wird.

Die Risikobereitschaft derjenigen, denen das Fällen einer Entscheidung übertragen worden ist, ist unterschiedlich ausgeprägt und kommt sehr häufig nicht explizit zum Ausdruck. Bei der Bewertung der durch die Entscheidung ausgelösten Wirkungen durch den Entscheidungsträger spielt sie jedoch eine zentrale Rolle. Dieser Einfluss ist 
umso größer, je höher das zu erwartende Risiko bei ungeklärter Risikoverteilung ist.

Es ist nicht nur das Bedürfnis der Führungskräfte und der Mitarbeiter nach Sicherheit, das die Geschäftsführung veranlasst festzulegen, wer welches Maß an Risiko trägt. Diese Festlegung ist auch im Interesse des Krankenhauses, weil ohne diese Klärung entweder Entscheidungsträger allzu hohe Risiken eingehen oder Entscheidungen möglicherweise nur mit erheblicher zeitlicher Verzögerung, eventuell überhaupt nicht gefällt werden.

Die Festlegung der Risikoverteilung ist schließlich auch im Interesse der Stakeholder. Für den Fall, dass Bedürfnisse von Stakeholdern nicht befriedigt werden, weil sie entwe- der bei Entscheidungen nicht berücksichtigt wurden oder eine Entscheidung nicht die gewünschten Folgen zeitigt, muss der Stakeholder wissen, wen er - wenn er das Risiko nicht tragen muss - zur Deckung der nicht erwünschten Folgen in Anspruch nehmen kann.

Das Risiko für die Stakeholder, unerwünschte Handlungsfolgen tragen zu müssen, kann minimiert werden, indem die Geschäftsführung den Handlungsspielraum der Entscheidungsträger gegebenenfalls einschränkt. Es ist zu fordern, dass für die Behandlung von Patienten medizinische Standards verbindlich vorgegeben werden, die in Abhängigkeit von bestimmten Faktoren den Charakter von Empfehlungen, Leitlinien oder Richtlinien haben. 